University of New Mexico

UNM Digital Repository

Summer 7-24-2020

\title{
SECONDARY ELECTRON YIELD MEASUREMENTS ON MATERIALS OF INTEREST TO VACUUM ELECTRON COMMUNICATION DEVICES
}

Talal Ahmed Malik

Follow this and additional works at: https://digitalrepository.unm.edu/ece_etds

Part of the Electrical and Computer Engineering Commons

\section{Recommended Citation}

Malik, Talal Ahmed. "SECONDARY ELECTRON YIELD MEASUREMENTS ON MATERIALS OF INTEREST TO VACUUM ELECTRON COMMUNICATION DEVICES." (2020). https://digitalrepository.unm.edu/ece_etds/ 489

This Thesis is brought to you for free and open access by the Engineering ETDs at UNM Digital Repository. It has been accepted for inclusion in Electrical and Computer Engineering ETDs by an authorized administrator of UNM Digital Repository. For more information, please contact amywinter@unm.edu, Isloane@salud.unm.edu, sarahrk@unm.edu. 
Talal Ahmed Malik

Candidate

Electrical and Computer Engineering Department

This thesis is approved, and it is acceptable in quality and form for publication:

Approved by the Thesis Committee:

Dr Mark Gilmore , Chairperson

Dr Salvador Portillo

Dr Edl Schamiloglu 
SECONDARY ELECTRON YIELD MEASUREMENTS ON MATERIALS OF INTEREST TO VACUUM ELECTRON COMMUNICATION DEVICES

\author{
by
}

TALAL AHMED MALIK

\title{
B.S,ELECTRICAL (TELECOM), UNIVERSITY OF ENGINEERING \& TECHNOLOGY TAXILA,2011
}

\author{
THESIS \\ Submitted in Partial Fulfillment of the \\ Requirements for the Degree of
}

\section{Master of Science \\ Electrical Engineering}

The University of New Mexico

Albuquerque, New Mexico

July 2020 


\section{DEDICATION}

To five people, I dedicate this accomplishment, all of whom made possible the journey that resulted in my master's degree.

To my parents, for their support and love during difficult times,

To my wife, for encouraging and supporting me whenever I felt low,

And to my host family, Jim and Jean Maka, for helping me to settle in and adapt to a new

culture. My appreciation for their support and care throughout my master's degree program is something I cannot express in words. 


\section{ACKNOWLEDGMENTS}

To my adviser, Dr. Mark Gilmore, I express my deepest gratitude for giving me the opportunity to be the part of MURI (Multidisciplinary University Research Initiative) project, for his continuous support and help, and for technical advice when I needed it. I also want to thank Dr Mark for giving me the freedom to make mistakes, to explore and learn new things in the experimental world.

Sincere thanks also are deserved to all of the members of my project, and especially to, Dr.Sal Portillo and Dr. Edl Schamiloglu, for your words of encouragement, for your patience, for your insightful comments on the result of my work and for taking time to serve on my committee. I could not have done much work without the support of Drs. Mark, Sal and Edl.

I am also thankful to the other project members, Dr. Ivana Gonzales, Dr. Ryan Johnson, Dr. Charles Jerald and Raul Gutierrez for participating in productive discussions during our meetings and for providing valuable comments. A special thanks to Dr Ivana for reviewing the SEY simulation portion of my thesis.

To all of my lab fellows, I thank you all, for creating a positive work environment and especially to Robert, Nathan, and Thomas for helping me with the physical work on this project. To Dr Sal's lab students - Joe, Robert and Ian - thank you for lending a helping hand whenever I asked. 
I wish to acknowledge the help and guidance provided by ECE staff members, especially Ma’am Yvone Nelson and Ma'am Cornelia Platero in academic affairs.

To the MURI team's commercial aerospace partners, Boeing and L3 Technologies, thank you for providing us with the list of materials and for advising us on cleaning protocols.

I'm very much thankful to Sir Amer and Sir Raheem for encouraging me to apply to Fulbright program.

And to the Fulbright Commission and State Department, thank you for selecting me from a large pool of candidates and giving me the opportunity to study in United States. 


\title{
Secondary Electron Yield Measurements on Materials of Interest to Vacuum Electron Communication Devices
}

by

\section{Talal Ahmed Malik}

B.S., Electrical (Communication Engineering), U.E.T Taxila, 2011 M.S., Electrical Engineering, University of New Mexico, 2020

\begin{abstract}
Microwave vacuum electron devices are a critical part of communication satellite payloads. The phenomenon of multipactor breakdown (MPB) can cause the performance of these devices to deteriorate, including their partial or total destruction. MPB depends on a number of factors, including the secondary electron yield (SEY) of materials used within the device. The process of electron multiplication, which leads to breakdown, initiates with the impact of primary electrons on target surfaces, and the resonance of free electrons within an oscillating electromagnetic field under vacuum conditions. Therefore, it is of interest to find materials and surface treatment methods that exhibit low SEY. This thesis work describes controlled measurements of SEY from electron bombardment of several materials in the low energy regime, from $10 \mathrm{eV}$ to $1 \mathrm{keV}$. Materials studied include copper $(\mathrm{Cu})$, silver, stainless steel, aluminum 6061 (Al), monel, nickel/cobalt and invar. In addition, different surface treatment methods employed in this study are described, which illustrate that SEY is a surface-specific process.
\end{abstract}




\section{Contents}

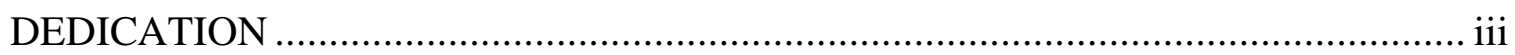

ACKNOWLEDGMENTS ......................................................................... iv

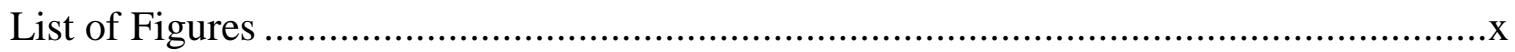

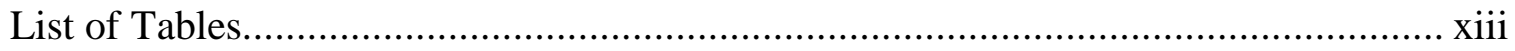

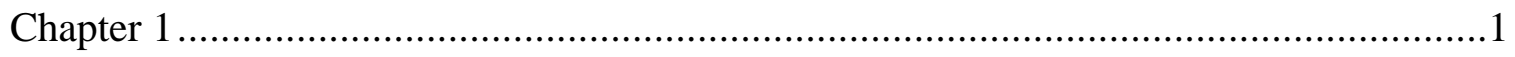

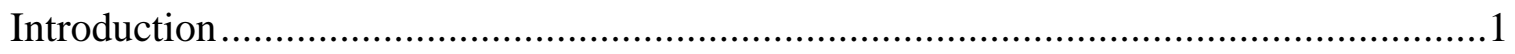

1.1 Multipaction and Secondary Electron Yield ........................................

1.2 Theory and Background of Multipactor Effect.................................................2

1.2.1 Mathematical Interpretation of Multipaction:..........................................4

1.3 Electron Emission..................................................................................

1.4 Secondary Electron Yield ..............................................................

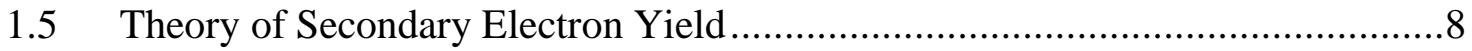

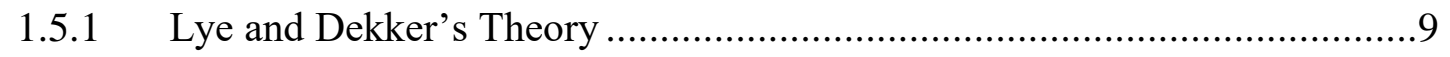

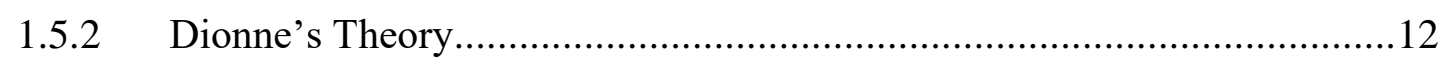

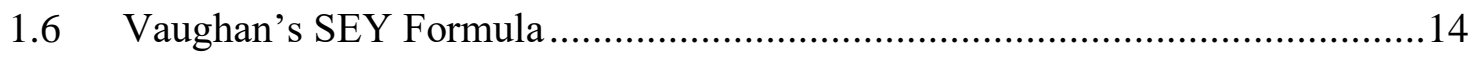

$1.7 \quad$ RF Satellite Devices ........................................................................ 15

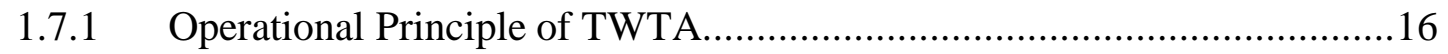

1.7.2 Operational Principle of Klystron .................................................. 17

1.8 Difference between the TWTA and the Klystron ......................................19

1.9 Measuring Secondary Electron Yield..................................................20 
1.10 SEY experiment at LNF Laboratory .............................................21

1.11 DFT-MC-Based Modeling of Secondary Electron Yield ................................22

1.12 Monte Carlo Simulation ....................................................................23

1.13 Comparison of Experimental Parameters ..................................................25

1.14 Scope and Organization of Thesis ........................................................27

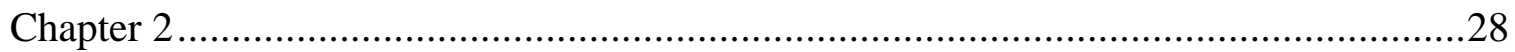

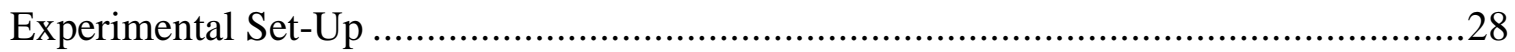

$2.1 \quad$ Vacuum System...............................................................................28

2.1.1 Establishing Ultra-High Vacuum .....................................................29

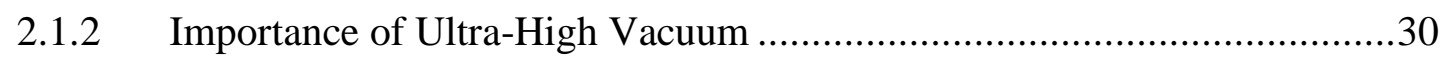

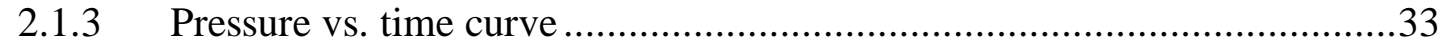

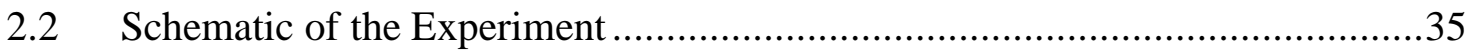

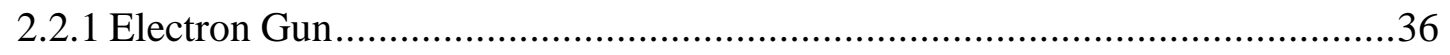

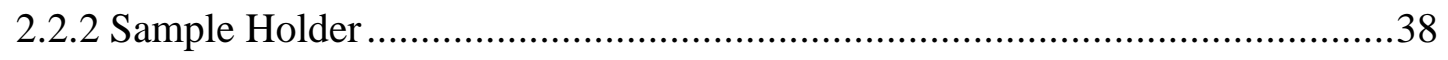

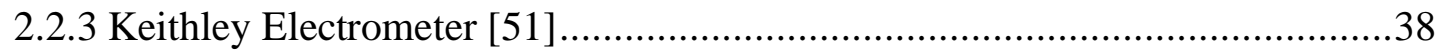

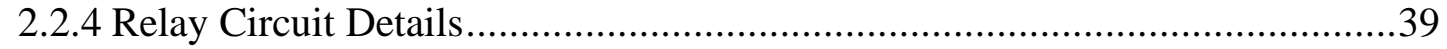

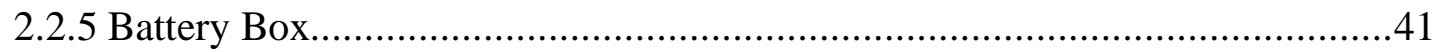

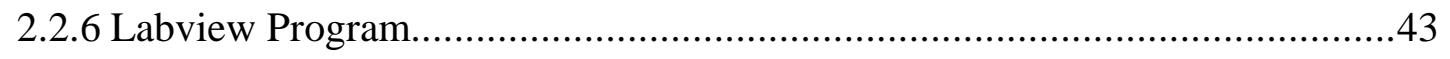

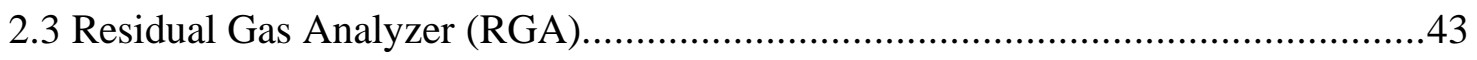

2.4 Heater and Temperature Measurements ...........................................................44

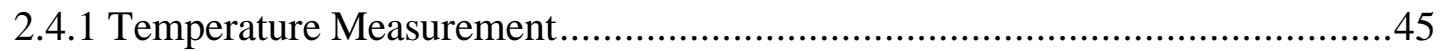

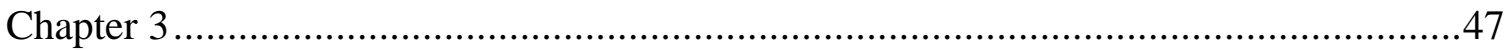

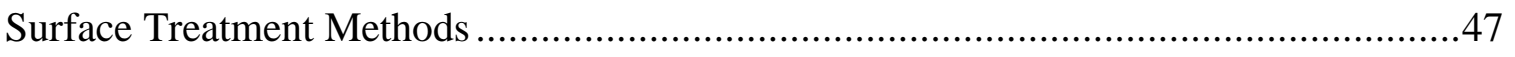

3.1 Samples Tested and Installation of Samples ..........................................47 


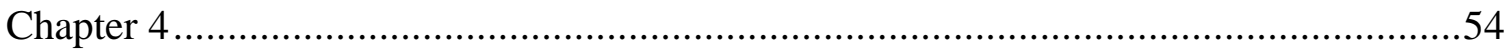

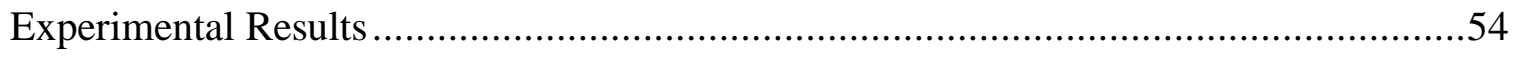

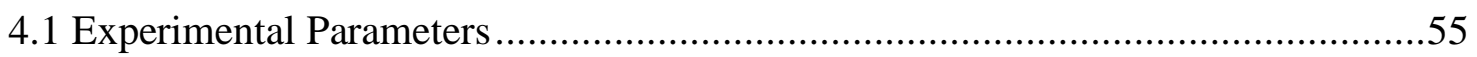

4.2 Materials and Cleaning Protocol Checklist ……….............................................57

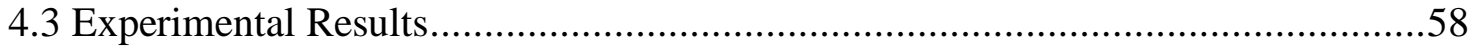

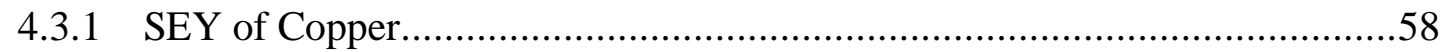

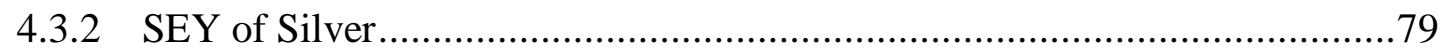

4.3.3 SEY comparison of Ultrasonic-I cleaned alloys...........................................8

4.3.4 Comparison of ultrasonic-I and roughed + methanol ................................8

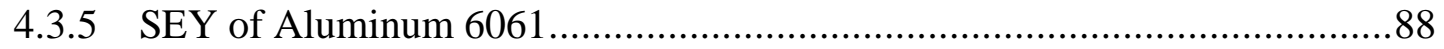

4.4 Comparison of experimental data with the "Universal Law" Model ......................92

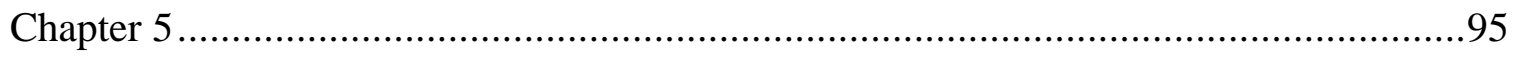

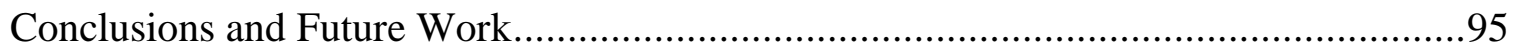

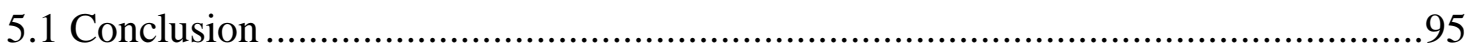

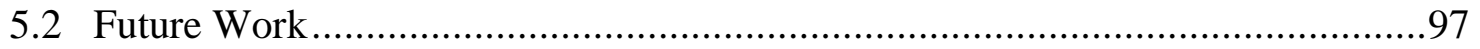

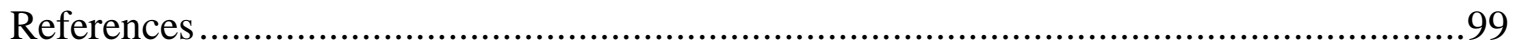




\section{List of Figures}

FIGURE 1: ILLUSTRATION OF THE MULTIPACTION EFFECT [11] ............................................. 4

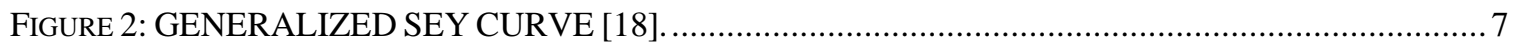

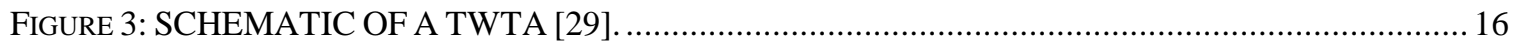

FIGURE 4: COMPONENTS OF A KLYSTRON AMPLIFIER [31] ................................................... 18

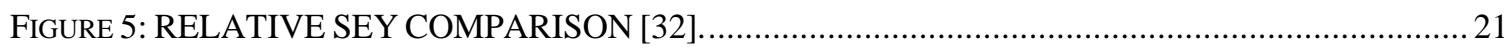

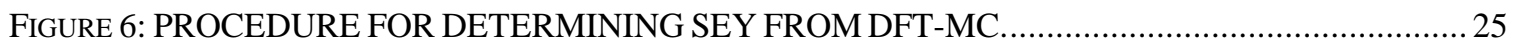

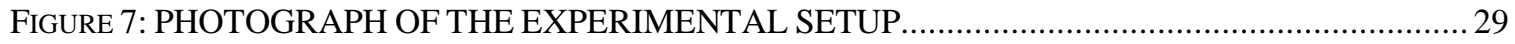

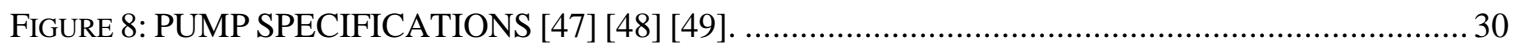

FIGURE 9: PRESSURE VS. TIME CURVE AS MEASURED BY ION GAUGE. ...................................... 34

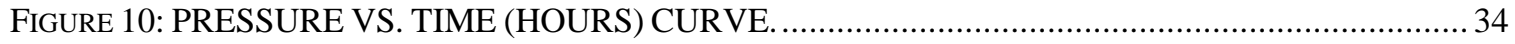

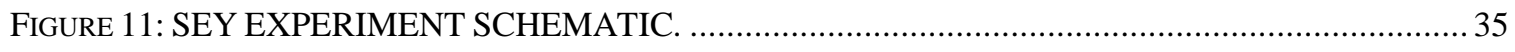

FIGURE 12: SCHEMATIC OF RELAY AND TRIGGER CIRCUIT USED FOR SWITCHING BATTERY

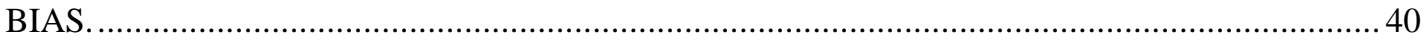

FIGURE 13: LTSPICE SIMULATION OF RELAY AND TRIGGER CIRCUIT...................................... 41

FIGURE 14: BLOCK DIAGRAM OF THE BATTERY BIAS CIRCUIT. ..................................................... 42

FIGURE 15: 3D VIEW OF THE CHAMBER INCLUDING THE HEATER USED FOR SAMPLE BAKING.

FIGURE 16: PHOTOGRAPH OF THE QUARTZ LAMP HEATER ASSEMBLY .................................... 45

FIGURE 17: 2.75" CF FLANGE THERMOCOUPLE FEEDTHROUGH. ................................................. 46

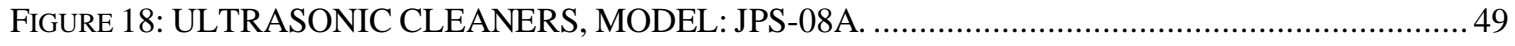

FIGURE 19: PHOTOGRAPH OF THE VACUUM CHAMBER WITH QUARTZ LAMP TURNED ON. ... 52

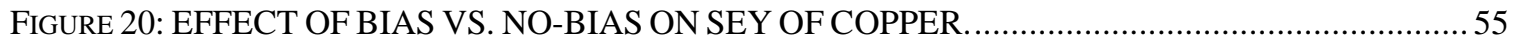

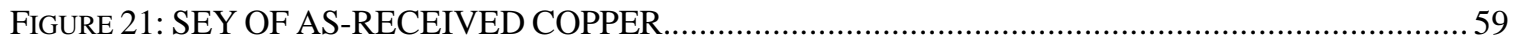

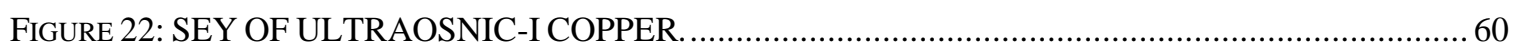

FIGURE 23: SEY OF COPPER WITH ULTRASONIC-I + 500 EV OF BEAM CONDITIONING.............. 61

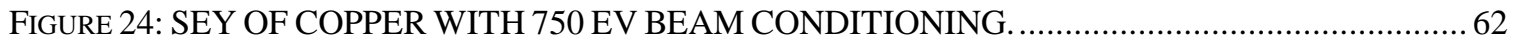

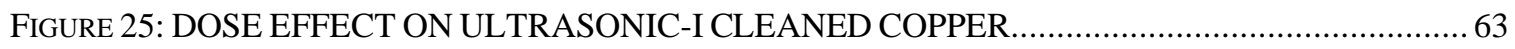


FIGURE 26: VARIATION IN SEY WITH DIFFERENT ANGLES OF INCIDENCE. 64

FIGURE 27: COMPARISON OF ULTRASONIC-I AND ULTRASONIC-II PROTOCOLS. 65

FIGURE 28: TEMPERATURE VS. TIME CURVE MEASURED BY A CONTACT THERMOCOUPLE ON THE SAMPLE. MEASUREMENT ACCURACY IS APPROXIMATELY $2^{\circ} \mathrm{C}$. .66

FIGURE 29: SEY OF COPPER WITH ULTRASONIC-I CLEANED AND BAKED TO 300 C. 67

FIGURE 30: SEY OF ULTRASONIC-I CLEANED COPPER + BAKED TO $300^{\circ} \mathrm{C}$ WITH BEAM ENERGY SCANNED UP TO $1980 \mathrm{EV}$. .68

FIGURE 31: SEY OF ULTRASONIC-II CLEANED AND BAKED TO $300^{\circ} \mathrm{C}$ COPPER SAMPLES C WITH BEAM ENERGY SCANNED UP TO 1000 EV. 69

FIGURE 32: SEY OF ULTRASONIC-II CLEANED COPPER + BAKED TO $300^{\circ} \mathrm{C}$ WITH BEAM ENERGY SCANNED UP TO $1980 \mathrm{EV}$ .70

FIGURE 33: VARIATION IN SEY OF COPPER WITH DIFFERENT SURFACE TREATMENTS. .71

FIGURE 34: SEY COMPARISON OF ULTRASONIC-II CLEANED AND BAKED AT THE TEMPERATURES INDICATED. .72

FIGURE 35: REPEATABILITY IN SEY DATA OF COPPER AFTER 12 HOURS OF HEATING BEING TURNED OFF. .73

FIGURE 36: REPEATABILITY IN SEY DATA OF COPPER AFTER 1,2,3,4,5 AND 6 HOURS OF HEATING BEING TURNED OFF. .74

FIGURE 37: COMPARISON OF THE SEY OF AS-RECEIVED COPPER SAMPLE \# 1 BAKED AT VARIOUS TEMPERATURES. .75

FIGURE 38: COMPARISON OF THE SEY OF AS-RECEIVED COPPER SAMPLE \# 2 BAKED AT VARIOUS TEMPERATURES. 76

FIGURE 39: SEY COMPARISON OF AIR EXPOSED COPPER WITH AND WITHOUT BAKING.......... 77 FIGURE 40: COMPARISON OF AS-RECEIVED COPPER BAKED IN AIR AND IN VACUUM.............. 78 FIGURE 41: 10 SHOTS OF SEY OF ULTRASONIC-I CLEANED SILVER.......................................... 80

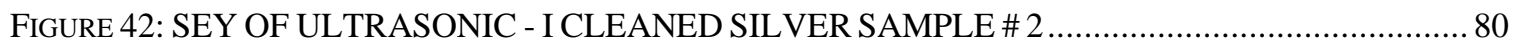
FIGURE 43: SEY COMPARISON OF SILVER SAMPLE WITH ASCENDING AND DESCENDING BEAM ENERGY...... .81 
FIGURE 44: VARIATION IN SEY OF SILVER WITH DIFFERENT SURFACE TREATMENTS. 82

FIGURE 45: COMPARISON OF AS-RECEIVED AND ULTRASONIC-I, II + BAKED SILVER SAMPLES.

FIGURE 46: SEY COMPARISON OF ALLOYS WITH ULTRASONIC-I CLEANING. 85

FIGURE 47: COMPARISON OF THE ULTRASONIC-I AND ROUGHED+METHANOL CLEANED

INVAR AND SILVER SAMPLES. 86

FIGURE 48: COMPARISON OF ULTRASONIC-I AND ROUGHED+METHANOL CLEANED STAINLESS STEEL AND COPPER SAMPLES. .87

FIGURE 49: SEY COMPARISON OF AS-RECEIVED ALUMINIUM SAMPLES.................................. 88

FIGURE 50: SEY OF AS-RECEIVED ALUMINIUM SAMPLE BAKED TO $300^{\circ} \mathrm{C}$. .89

FIGURE 51: SEY OF AS-RECEIVED ALUMINIUM WITH BEAM ENERGY SCANNED UP TO 1980 EV. .90

FIGURE 52: SEY COMPARISON OF ALUMINIUM WITH DIFFERENT SURFACE TREATMENTS.... 91 FIGURE 53: CURVE FITTING SEY EXPERIMENTAL DATA OF COPPER WITH UNIVERSAL LAW CURVE. .93

FIGURE 54: CURVE FITTING SEY EXPERIMENTAL DATA OF COPPER WITH UNIVERSAL LAW CURVE. .94 


\section{List of Tables}

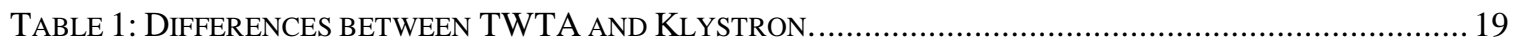

TABLE 2: COMPARISON OF DIFFERENT SEY EXPERIMENTAL PARAMETERS. ................................................ 25

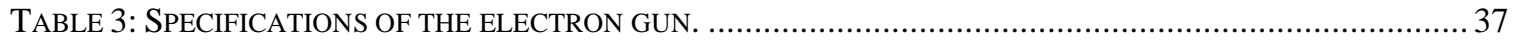

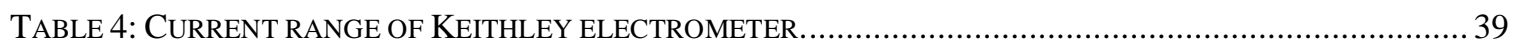

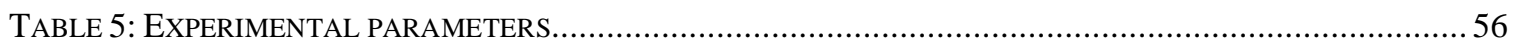

TABLE 6 : CHECKLIST FOR MATERIALS AND CLEANING PROTOCOLS EMPLOYED IN THE SEY EXPERIMENT.... 57

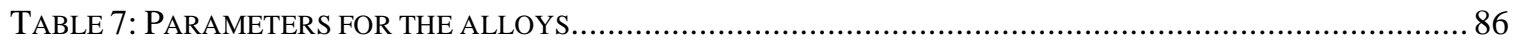




\section{Chapter 1}

\section{Introduction}

\subsection{Multipaction and Secondary Electron Yield}

Microwave vacuum electron-based devices (MVED's), such as Traveling Wave Tube Amplifiers (TWTA) and Klystron Amplifiers, installed in the communication payload subsystem of satellites are susceptible to the phenomenon of multipactor breakdown (MPB) [1][2]. In this phenomenon, an electron avalanche process takes place in a high vacuum environment under the influence of RF fields. MPB is a function of a combination of many factors, such as operating frequency, input power, surface geometry, direction and magnitude of the RF field, and secondary electron yield (SEY) of electrode materials [3][4]. SEY is one of the important factors causing MPB.

Power and bandwidth are considered precious commodities in telecommunication services and a link budget for satellite communication is calculated on the basis of the output power generated by VEDs at a specified frequency of operation. Key parameters of the VEDs, like output power, bandwidth, noise figure, gain, and efficiency, can become significantly impacted due to MPB [4][5][6][7]. In a worst-case scenario, MPB can also lead to physical damage of the device, such as electrode melting due to heating or a thermal cracking of the output RF window [3][4][6][8].

The maximum output power from a satellite transponder is limited since MPB itself requires power to sustain, which leads to degraded output power performance by the 
amplifier. In the case of TWTAs, the overall efficiency is dependent on the performance of the depressed collector. As the electron beam is recovered by the collector electrodes, it can generate secondary electrons which escape the collector and thereby degrade performance, resulting in a less efficient TWTA with higher electron back streaming. Back streaming electrons not only reduce the TWTA efficiency, but they also result in higher gain and frequency variation, which impact the overall performance of the communication link. Therefore, the overall data rate of the communication link could be significantly improved by using materials with low SEY. Thus, for space-based applications, efficient and reliable multipactor-free operation is important for supporting space communication links.

\subsection{Theory and Background of Multipactor Effect}

Multipaction was first observed in 1924 by Camille Gutton, a French physicist in Nancy. Detailed study was undertaken by other scientists, including Philo Fransworth, who wanted to utilize and take advantage of the amplified effect of this phenomenon [9]. Multipaction is an electron resonance effect that occurs when RF fields accelerate electrons in a vacuum and cause them to impact with a surface, which, depending on its energy, releases one or more electrons into the vacuum. These electrons can then be accelerated by the RF fields and impact with the same or another surface. When the impact energies, number of electrons released, and timing of the impacts are such that a sustained multiplication of the number of electrons occurs, the phenomenon will grow exponentially and may lead to operational problems. In RF space systems, multipaction will cause loss/distortion of the $\mathrm{RF}$ signal (increase of noise figure or bit-error-rate) and can damage RF components or subsystems due to excess RF power being reflected back or dissipated. 
In accelerators, multipaction can produce electron clouds preventing the undisturbed buildup of accelerator energy levels. Multipaction can also cause an increase of local pressure which could lead to a more destructive corona breakdown [10].

Existence of multipaction in a vacuum container (Fig. 1) is dependent upon the following conditions:

a) The phenomenon occurs in a vacuum or near vacuum environment, as free electrons in open atmosphere collide with air or dust particles, reducing their kinetic energy and thus the potential to release secondary electrons upon collision. The distance between the opposing surfaces should be smaller than the mean free path $^{1}$ for electrons, which is normally possible in good vacuum condition.

b) Free electrons ${ }^{2}$ are available or made available in the container. In a space environment, free electrons are emitted from the surfaces by incident high energy elemental particles, while in on-ground testing they are sourced by an electron gun.

c) The time taken by the electron to travel from the surface from which it was released to the surface it impacts with is to be an integer multiple of one half of the RF period (resonance).

d) The average number of electrons released is greater than one, which is dependent on the SEY of the surface, which in turn is dependent on the field strength (RF power) between the surfaces.[10]

\footnotetext{
${ }^{1}$ Definition of mean free path, http://physics.bu.edu/ redner/211-sp06/class-macromicro/kinetic_meanfreepath.html

${ }^{2}$ Free electrons: The electrons which are not attached to the nucleus of a atom and free to move when external energy is applied are called free electrons. http://www.physics-and-radioelectronics.com/electronic-devices-and-circuits/introduction/free-electrons.html
} 


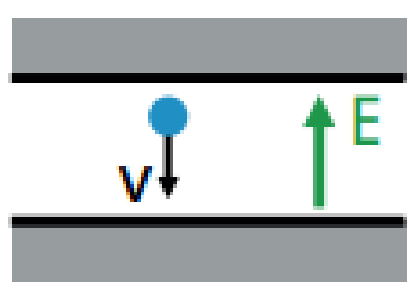

t1

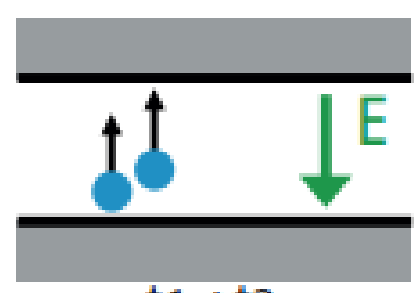

$\mathrm{t} 1<\mathrm{t} 2$

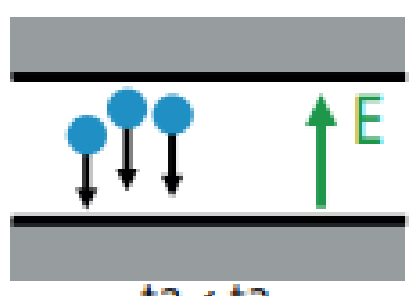

$\mathrm{t} 2<\mathrm{t} 3$

Figure 1: ILLUSTRATION OF THE MULTIPACTION EFFECT [11].

\subsubsection{Mathematical Interpretation of Multipaction:}

Mathematically, multipaction events are understood as a function of the input power to a component, according to Larmor's formula [12]:

$P=2 e^{2} a^{2} /\left[3\left(4 \pi \varepsilon_{o} c^{3}\right)\right]$

where,

$\mathrm{P}=$ the power generated by an accelerated charge,

$\mathrm{e}=$ the charge of an electron

$\varepsilon_{0}=$ the dielectric constant

$\mathrm{a}=$ the acceleration of the charge

$\mathrm{c}=$ the speed of light in a vacuum, or $3 \times 10^{8} \mathrm{~m} / \mathrm{s}$.

Higher values of dielectric constant, $\varepsilon_{0}$, will result in reduced power for the accelerated charged.

Similarly, mathematically the multipaction threshold voltage, $\mathrm{V}_{\mathrm{o}}$, can be found from the equation:

$$
V_{o}=(2 \pi d / \lambda)^{2}\left[m_{e} c^{2} / \pi e\right]
$$

where, 
$\mathrm{V}_{\mathrm{o}}=$ the acceleration voltage between charged surfaces

$\mathrm{m}_{\mathrm{e}}=$ the mass of an electron

$\lambda=$ the wavelength

$\mathrm{d}=$ the spacing between surfaces.

\subsection{Electron Emission}

Electrons exist in naturally bound states; they constantly spin in orbitals/shells at some specific distances from the nucleus. Electrons can move around in any direction while orbiting around the nucleus of the atom. In order to set an electron free, kinetic energy must be applied to it in its bound state so that the potential energy of the bond can be overcome. According to the way in which the electron receives its kinetic energy, four different processes of electron emission can be categorized as follows [13]:

\section{Thermionic Emission}

The process by which free electrons are emitted from the surface of a metal when external heat energy is applied is called thermionic emission [14]. This kind of emission occurs in metals that are heated to a very high temperature. When heat energy applied to the metal is increased to a higher value, the free electrons gain sufficient energy to overcome the attractive force of the atomic nucleus, which holds the free electrons in the metal. The free electrons, which overcome the attractive force of the nuclei, break the bonding with the metal and jump into the vacuum. The number of free electrons escaped from the metal depends on the amount of heat applied to the metal and the work function of the metal. 


\section{Photoemission}

Irradiation of a substance with electromagnetic radiation of sufficiently short wavelength, for instance with ultraviolet light or with X-rays, results in 'photoelectric emission' [13].

\section{Field Emission}

Application of significantly large electrostatic or quasistatic fields to the surface of the material results in field emission [15].

\section{Secondary Emission}

Bombardment of a substance or a material with particles, for example by atoms, ions or electrons lead to emission of secondary electrons [13].

\subsection{Secondary Electron Yield}

SEY takes place when a surface of material is bombarded by incident electrons. The SEY coefficient $\delta$ is defined as the ratio of total emitted secondary electron current to incident primary electron current, i.e. [16]:

$$
\delta=\mathrm{Is} / \mathrm{Ip}
$$

The relation between $\delta$ and electron beam energy forms the universal SEY curve shown in Fig. 2. Secondary electrons are generally classified as 'true' secondary electrons and backscattered electrons. Standard convention distinguishes between secondary electrons as those with energy below $50 \mathrm{eV}$ and backscattered electrons as those with higher energies, up to the incident energy. The backscattering current is generally smaller than the true secondary electron emission current by one order of magnitude in typical space environments [17]. 


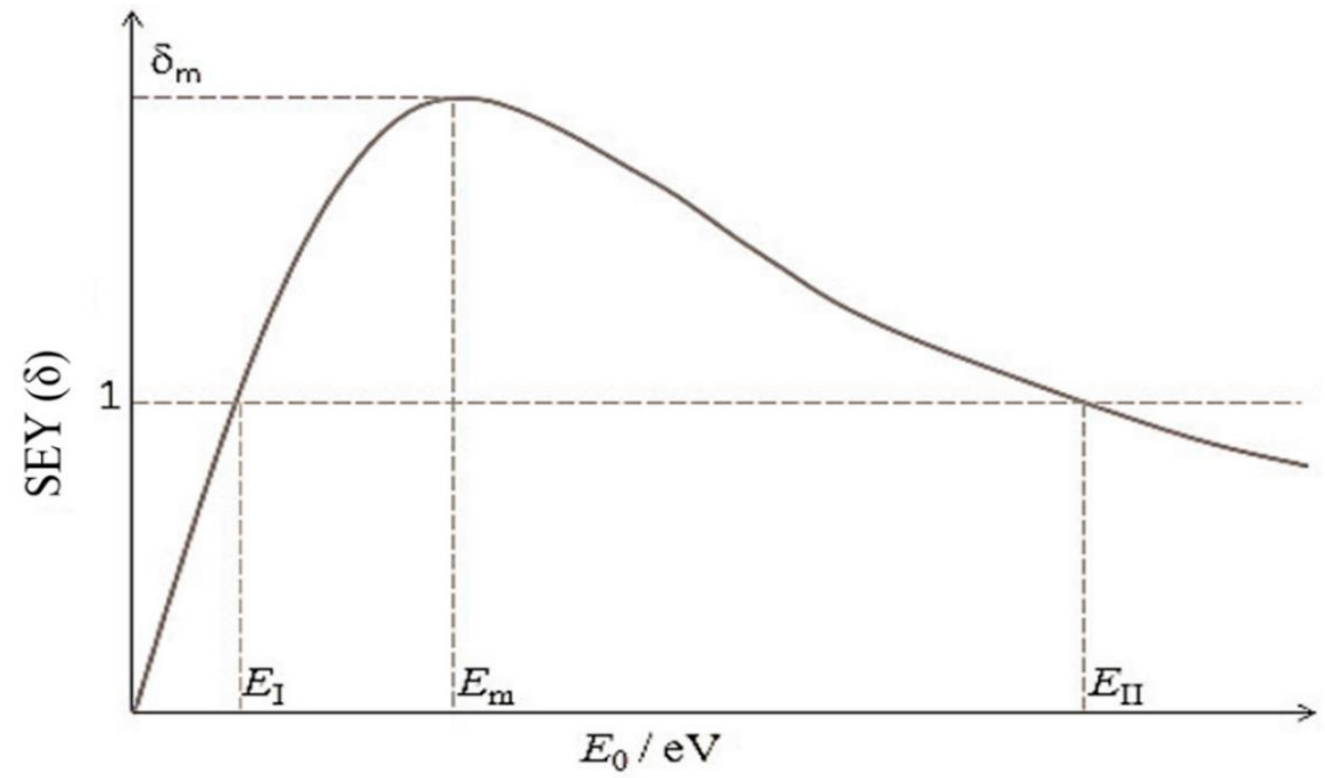

Figure 2: GENERALIZED SEY CURVE [18].

The number of secondary electrons produced per incident electron depends upon a number of factors, including the energy of the incident electrons, the angle of incidence of the electron beam, and the chemical composition and state of the metal surface.

Figure 2 above shows the generic SEY curve obtained when the SEY coefficient and the primary beam energy voltage are plotted. The SEY coefficient is plotted on the vertical axis while primary beam energy is plotted on the horizontal axis. It can be seen from the graph that an increasing primary beam energy increases the number of secondary electrons generated, but the depth at which the secondary electrons are emitted also increases and there is thus an increasing loss due to absorption.

The nature of the SEY curve can also be explained as follows. At low primary beam energies the secondary electrons produced in the material do not have sufficient energy to overcome the work function so that the SEY is lower at low primary beam energies. Similarly, the SEY is also lower at high beam energies because at high energies most 
secondary electrons are generated deep in the material and hence cannot overcome the work function of the target material, thus remaining inside the material [19].

Several parameters describe points of interest on the curve which is shown as $\delta_{\mathrm{m}}$ in Fig. 2. The maximum $\delta_{\mathrm{m}}$ varies from material-to-material and depends on the cleanliness or contamination present on the surface. Thus, SEY is considered to be a surface specific phenomenon. It will be shown experimentally in upcoming chapters how surface preparation influences SEY. The chemical state of the metal surface also plays an important role in determining the SEY for the respective metal. The SEY for metals that do not exceed 1.6 may experience an increase in the SEY with a contaminating layer on the surface well above 2 . The work function of the metal also depends upon what is actually residing on the surface of material.

$\mathrm{E}_{\mathrm{m}}$ represents the electron beam energy in $\mathrm{eV}$ at which the maximum SEY occurs. $\mathrm{E}_{1}$ and $\mathrm{E}_{\mathrm{II}}$ represent the first and second crossover point of energy, respectively. These energy points define the value of $\delta=1$. These points on the curve as explained above have experimental significance as the energy crossover points can be measured to have net zero electric current [20].

\subsection{Theory of Secondary Electron Yield}

Generation of secondary electrons from a surface is directly related to the amount of kinetic energy transferred by the striking or primary electron. As a result of the above, the SEY is measured as a function of the energy of the incident electron. The energy lost by primary electrons is a function of the depth of penetration achieved through absorption and or scattering [21]. Secondary electron emission is a complex phenomenon with a number of 
quantum and classical mechanics theories proposing to explain the phenomenon. However, none have been able to adequately explain details of this process for all materials and all energy levels. Below, we will briefly review the phenomenon as explained by leading theories.

\subsubsection{Lye and Dekker's Theory}

- Elementary theories of electron emission intentionally omit details of electronic excitation and escape mechanism, as this allows a semi-quantitative comparison with experimentation. The theory presented by Lye and Dekker, which is a generalization of Bruining's theory [22] for SEY, is mathematically represented as follows:

$$
\delta=\int_{0}^{\infty} n(x, E o) f(x) d x
$$

where,

$\mathrm{n}(\mathrm{x}, \mathrm{Eo}) \mathrm{dx}$, represents the number of secondary electrons produced per incident primary electron of initial energy Eo in a layer of thickness $\mathrm{dx}$ while being at a depth " $\mathrm{x}$ " below the surface; $f(x)$ is the probability of a secondary electron being produced at a depth of " $x$ " escapes from the surface.

The following assumptions were made by Lye and Dekker in this theory:

- $\mathrm{n}(\mathrm{x}, \mathrm{Eo})$ is proportional to the energy loss of the primary beam per unit of path length, $\mathrm{n}(\mathrm{x}, \mathrm{E} 0)=-\mathrm{KdE} / \mathrm{dx}$, assuming normal incidence with energy evaluated per particle. 
- The probability that a secondary electron produced and escaping from the surface is essentially given by $\exp (-\alpha \mathrm{x})$, where $1 / \alpha$ corresponds to the effective range of secondary electrons in the object under observation.

- Whiddington's law governs the primary energy losses, given by $\mathrm{dE} / \mathrm{dx}=-\mathrm{A} / \mathrm{E}(\mathrm{x})$, where $\mathrm{A}$ is the characteristic of the solid. A, $\alpha$, and $\mathrm{k}$ are fit parameters [22].

Baroody pointed out that, on the basis of these assumptions, a reduced SEY curve, independent of $\alpha, \mathrm{A}$, and $\mathrm{k}$ parameters could be deduced [23]. Thus, if $\delta \mathrm{m}$ represents the maximum yield for a primary energy electron $\mathrm{E}_{\mathrm{om}}$, then a plot of $\delta / \delta_{\mathrm{m}} \mathrm{vs}$. E/Eom yields a curve which is independent of the characteristics of the particular material.

Baroody used Whiddington's law and integrated Eq (1.4) to give the form of the universal SEY curve,

$$
\frac{\delta}{\delta_{m}}=\frac{1}{F(0.92)} F\left(0.92 E_{o} / E_{o m}\right),
$$

where,

$$
F(r)=\exp \left(-r^{2}\right) \int_{0}^{r} \exp \left(y^{2}\right) d y .
$$

Here $\delta_{\mathrm{m}}$ and $\mathrm{E}_{\mathrm{om}}$ are, respectively, the maximum value of secondary emission and primary voltage at which it occurs. The curve represented by the above equation deviates particularly near the extreme ends of the spectrum. Due to this discrepancy, it is suspected that Whittington's law may not describe the energy losses of the primaries properly. In actuality, the law gives a penetration depth which is proportional to the square of the primary energy. Whiddington's law gives a penetration depth proportional to the square of the primary energy, whereas Young [23] concludes from transmission measurements of 
electrons with energies between $0.3 \mathrm{keV}$ and $7.5 \mathrm{keV}$ through aluminum oxide films that the range-energy mathematical relationship for this material is given by

$$
R=0.0115 E o^{1.35}
$$

where,

$\mathrm{E}_{\mathrm{o}}$ is the initial primary energy expressed in $\mathrm{keV}$ and

$\mathrm{R}$ is the range expressed in $\mathrm{mg} / \mathrm{cm}^{2}$

The range listed above defines the mass per $\mathrm{cm}^{2}$ by which the electron beam has been stopped and is related to A and Eo as described by

$$
R=E o^{n+1} / A(n+1)
$$

Since the above equations are not an accurate predictors for secondary emission, Lye and Decker developed an elementary theory of secondary emission on the basis of the law for energy loss for primaries given by

$$
d E /_{d x}=-A / E^{n}(x)
$$

where $\mathrm{A}$ is the material characteristic, while $\mathrm{n}$ is an assumed power.

In light of the above results, it can be shown that:

$$
\delta=K[A(n+1) / \alpha]^{\frac{1}{n+1}} \exp \left(-r^{n+1}\right) \int_{0}^{r} \exp \left(y^{n+1}\right) d y=K\left[\frac{A(n+1)}{\alpha}\right]^{\frac{1}{n+1}} G(r)
$$

where $\mathrm{K}=$ constant and

$$
r^{n+1}=\propto R=\propto \frac{E o^{n+1}}{A(n+1)}
$$

When $n=0.35$, it is observed that the agreement with experimental curves for metals is considerably better than for the Baroody curve. Some contradictions were raised against 
the assumptions which were supported by experimental data of low energy electron dissipation of aluminum oxide carried out by Young [24].

\subsubsection{Dionne's Theory}

Dionne studied the effects of scattering on SEY [25]. Physical interpretation of SEY curve parameters were published in [26]. A brief summary of [25] is provided by Scholtz in [16]. In deriving $\delta\left(\mathrm{E}_{\mathrm{p}}\right)$, the following assumptions were made:

- The number of secondary electrons produced per unit length at position $\mathrm{x}$ is equivalent to the energy loss by the primary electrons divided by the energy required to produce one electron.

- The probability of migration for an excited electron is given by $\exp (-\alpha x)$, with $\alpha$ the inverse of the effective extension length and $x$ the depth at which the electrons are produced in the surface, respectively.

- The angular scattering observed by primary electrons is explained by the assumption that the general average loss of energy is independent of the material depth $\mathrm{x}$ and $\frac{d E}{d x}=-\frac{E p}{R}$. This assumption is defined as the "constant loss" assumption with $\mathrm{R}$ the penetration depth. $\mathrm{R}$ is determined by primary electrons which do not undergo angular scattering. In that case the energy loss of the primary electrons is given by the power law $\frac{d E}{d x}=-\frac{A}{E^{n-1}}$, where $\mathrm{n}$ is determined through experiments and is in the range $1.3-1.6$.

The above assumptions are similar to those made by Lye and Dekker. In addition, however, Dionne assumes 
- The effective escape probability for a secondary electron that reaches the surface is B. The SEY can then be written as

$$
\delta\left(E_{p}\right)=B \times \frac{E_{p}}{\Omega R} \times\left(1-e^{-\alpha R}\right) / \alpha,
$$

where range $\mathrm{R}$ and primary energy $\mathrm{E}_{\mathrm{p}}$ are related by :

$$
R=\frac{E_{p}^{n}}{A n} .
$$

The above is also true after considering the scattering of secondary electrons as they are generated in massive quantities. According to Dionne, $E_{o c}^{1}$ is given as

$$
E_{o c}^{1}=0.51 E m \delta_{m}^{-1.32},
$$

where $\mathrm{E}_{\mathrm{m}}$ is the primary energy leading to maximum yield and $\delta m$ is the coefficient of the maximum SEY.

The conclusion obtained by Dionne is described by

$$
\delta=(B / \xi)(A n / \alpha)^{1 / n}(\alpha d)^{1 / n-1}\left(1-e^{-\alpha d}\right),
$$

where,

$$
\begin{aligned}
& \xi=\text { excitation energy of secondary electrons } \\
& \mathrm{B}=\text { escape probability } \\
& \mathrm{A}=\text { constant for primary electron absorption } \\
& \alpha=\text { absorption constant for secondary electron absorption } \\
& \mathrm{n}=\text { power-law exponent } \\
& \mathrm{d}=\text { maximum penetration depth } \\
& \text { with } \mathrm{n}=1.35 \\
& \text { Eom }=2.3(\mathrm{~A} / \alpha)^{0.74} \\
& \text { Eom }=2.3(\mathrm{~A} / \alpha)^{0.74}
\end{aligned}
$$




$$
\begin{aligned}
& \delta m=0.9(\mathrm{~B} / \zeta)(A / \alpha))^{0.74} \\
& E_{0 C}^{I I} \cong 2.36[(\mathrm{~B} / \zeta)(A / \alpha)]^{2.86} .
\end{aligned}
$$

The above equations lead to the following conclusions:

$\alpha \propto$ electrical conductivity

A $\propto$ density of the material $\rho$

$\mathrm{B} \propto(1-\mathrm{r})$, where the value of $\mathrm{r}$ depends upon the physical conditions of the material and is the defined as the reflection coefficient.

$\zeta \propto \phi$, where $\phi$ is the work function of the metal

$\chi+E g$ where $\chi$ is the electron affinity and $\mathrm{E} g$ is the band gap for semiconductor or insulator [26].

\subsection{Vaughan's SEY Formula}

Since Dekker and Lye formulas for SEY do not agree at lower energies, Vaughan proposed a formula that incorporates the effect of angular incidence of the electron beam on the SEY of the metal surface [20]. These are empirical-based formulas that are derived from the experiment conducted by Gibbon and Ritz [20]. The formulas proposed by Vaughan were modified for the clean sample after more accurate experiments by Shih and Hor [27]. The formulas excerpted from [28] are

$$
\begin{aligned}
& \mathrm{V}_{\text {max }}(\varphi)=\mathrm{V}_{\text {max }}(0)\left(1+\mathrm{Ksv} \varphi^{2} / 2 \pi\right) \\
& \delta_{\text {max }}(\varphi)=\delta_{\text {max }}(0)\left(1+\mathrm{Ks} \delta \varphi^{2} / 2 \pi\right)
\end{aligned}
$$

In the equations above the $\mathrm{K}$ factors refer to the smoothness of the surface and can be considered as 1 for the surface in vacuum. A high value of $\mathrm{K}$ is taken for a surface which 
is clean and free from any kind of contamination. The value of $\mathrm{K}$, therefore, depends on how clean the surface of sample is.

The mathematical form is

$\delta(\theta) / \delta_{\max }(\theta)=\left(\mathrm{ve}^{1-\mathrm{v}}\right)^{\mathrm{k}}$

$\mathrm{k}=\mathrm{k}_{1}=0.56, \quad$ for $\mathrm{v}<1$

$\mathrm{k}=\mathrm{k}_{2}=0.25, \quad$ for $1<\mathrm{v} \leq 3.6$

$\delta(\theta) / \delta_{\max }(\theta)=1.125 / \mathrm{v}^{0.35}$ for $\mathrm{v}>3.6$

and $\mathrm{v}=\left(\mathrm{V}_{\mathrm{i}-\mathrm{V}_{\mathrm{o}}}\right) /\left(\mathrm{V}_{\max }(\theta)-\mathrm{V}_{\mathrm{o}}\right)$,

where Vo is the minimum voltage at which the secondary electrons are generated and $\theta$ (in radians) is the direction of incident primary beam relative to the surface normal.

\subsection{RF Satellite Devices}

Radio frequency devices are essential components of satellite communication payloads. High power devices are required to be placed in the transmit path of the satellite communication link to manage varying power supply over the entire energy spectrum. Due to their specific usage characteristics, these devices are prone to high power related failure phenomena, including, but not limited to passive inter-modulation, harmful effects due to multipactor, and corona discharge. Multipactor discharge may generate noise and may reduce the output power by increasing the loss due to mismatch, thereby increasing the device's temperature.

The RF VEDs of most interest to satellite communication are:

1) TWTA (Travelling Wave Tube Amplifier) 
2) Klystron Amplifier.

The working principle of the above-mentioned RF devices are discussed below.

\subsubsection{Operational Principle of TWTA}

The TWT is an electron tube used for amplification at microwave frequencies [29]. At microwave frequencies the conventional circuit theory concepts no longer apply and it is required to use electromagnetic theory to describe the electric and magnetic fields that exist in electromagnetic waves. The operation of the TWT depends on the interaction of a beam of electrons with an electromagnetic wave. The internal physical composition of a TWTA is shown in Fig. 3.

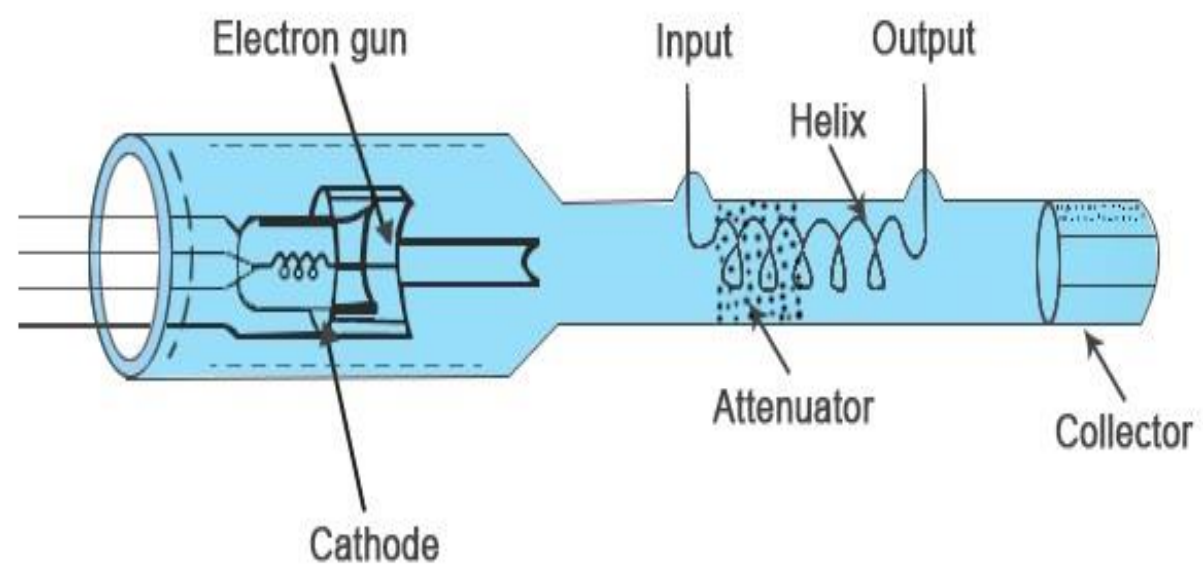

Figure 3: SCHEMATIC OF A TWTA [29].

The TWTA is made up of a vacuum tube with an electron gun installed at one end of the tube under the influence of magnetic field. The cathode in the electron gun ejects electrons upon heating. The RF port in the beginning of the helix structure is used to input the RF signal which travels at the speed of light and gets slowed down due to traveling along the 
helix structure. The geometry of the helix is referred to as a slow wave structure that is responsible for decreasing the axial speed of the electromagnetic wave. The shape of the helix slows the effective velocity of the wave along the common axis of the helix and the tube to about one-tenth the speed of light.

The electron beam transfers its energy to the input RF signal through the process of velocity modulation on its way to the collector. Velocity modulation is caused by the interaction between the traveling-wave fields and the electron beam. Bunching causes the electrons to give up energy to the traveling wave if the fields are of the correct polarity to slow down the bunches. The energy from the bunches increases the amplitude of the traveling wave in a progressive action that takes place all along the length of the TWT.

As shown in Fig. 3 , the electron beam is directed down the center of the helix while, at the same time, an RF signal is coupled onto the helix. The electrons of the beam are velocitymodulated by the electric fields produced by the RF signal. Amplification begins as the electron bunches form and release energy to the signal on the helix. The slightly amplified signal causes a denser electron bunch which, in turn, amplifies the signal even more. The amplification process is continuous as the RF wave and the electron beam travel down the length of the tube [30].

\subsubsection{Operational Principle of Klystron}

Klystron amplifiers are used in a variety of industries, including the space industry. They are widely used in satellite communications like the TWTA. The klystron is a device for amplifying microwave frequency signals that achieves high levels of power gain by applying vacuum tube principles and the concept of electron bunching. Figure 4 shows the components used in the construction of a klystron. 


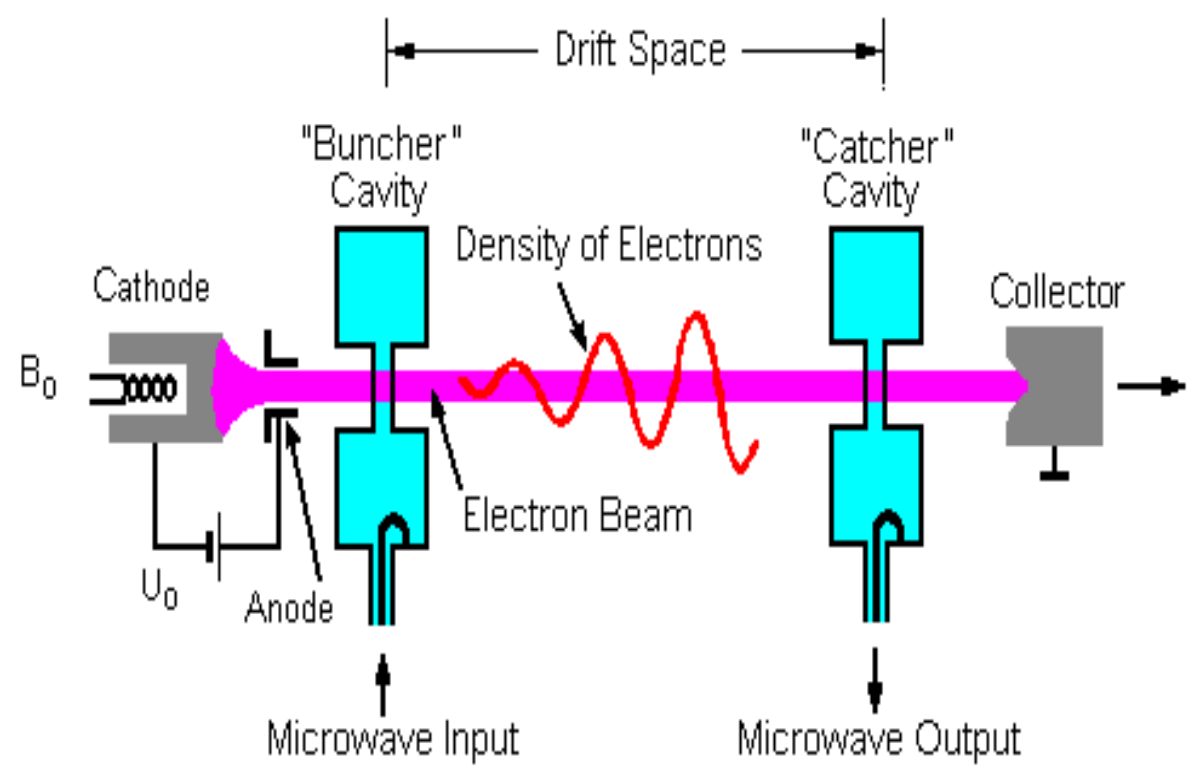

Figure 4: COMPONENTS OF A KLYSTRON AMPLIFIER [31].

The simplest klystron tube is the two-cavity klystron, as shown in Fig. 4. In this tube there are two microwave cavity resonators, the "buncher" and the "catcher".

At one end of the tube is the hot cathode which produces electrons when heated by a filament. The electrons are attracted to and pass through an anode cylinder at a high positive potential. The cathode and anode act as an electron gun to produce a high velocity stream of electrons.

The beam first passes through the buncher cavity resonator through grids attached to each side. The buncher grids have an oscillating AC potential across them produced by standing wave oscillations within the cavity excited by the input signal at the cavity's resonant frequency applied by a coaxial cable or waveguide. The electrons then pass through a second cavity, called the catcher, through a similar pair of grids on each side of the cavity. The function of the catcher grids is to absorb energy from the electron beam. The bunches of electrons passing through excite standing waves in the cavity that have the same resonant 
frequency as the buncher cavity. Electrons do work on the electric field and are decelerated; their kinetic energy is converted to electric potential energy, increasing the amplitude of the oscillating electric field in the cavity. Thus, the oscillating field in the catcher cavity is an amplified copy of the signal applied to the buncher cavity. The amplified signal is extracted from the catcher cavity through a coaxial cable or waveguide [31].

\subsection{Difference between the TWTA and the Klystron}

Differences between the TWTA and the Klystron are listed in Table 1.

Table 1: Differences between TWTA and Klystron.

\begin{tabular}{|l|l|}
\hline Klystron & TWTA \\
resonant cavity. & on a helix structure. \\
\hline Narrow operational bandwidth. & Wide operational bandwidth. \\
\hline Low output power generation. & High output power generation. \\
\hline $\begin{array}{l}\text { Interaction between electron and } \\
\text { RF field only takes place at the }\end{array}$ & Interaction between electron and \\
gaps of resonant cavity. & entire length of tube. \\
\hline No coupling between the & In a coupled cavity TWT, \\
resonant cavities. & coupling takes place between \\
& cavities. \\
\hline High efficiency. & Low efficiency. \\
\hline
\end{tabular}




\subsection{Measuring Secondary Electron Yield}

Important elements needed in experiments for measuring SEY are as follows:

- Electron producing source, i.e., electron gun.

- Sample from which SEY is to be measured.

- Measurement method to determine beam and target currents for determining SEY coefficient.

We will discuss a couple of experimental methods for measuring SEY below.

\section{a) Calculating SEY using a spherical shell}

In this method of measurement, the sample to be tested is placed inside a spherical shell. An electron beam is used to target the sample with incident primary electrons. Secondary electrons generated on the sample surface are captured by the shell surrounding the sample by applying a suitable bias voltage. The inner wall of the spherical shell is coated with low SEY material to avoid generation of tertiary electrons [22].

\section{b) SEY calculation by ratio of currents}

In this method of SEY measurement, two currents are measured, the incident primary beam current and the target current. The primary beam current $(\mathrm{Ib})$ is calculated by applying positive bias voltage to the sample and zero or negative bias to the metal surface to calculate the target current (It). The primary beam current and target current are calculated against different beam energy levels and SEY is mathematically computed as

$$
\operatorname{SEY}(\delta)=1-\mathrm{It} / \mathrm{I}_{\mathrm{b}}
$$

This method of calculation has been used in this thesis for determining SEY. 


\subsection{SEY experiment at LNF Laboratory}

The National Laboratory of Frascati (LNF) is a prestigious European lab located in Italy which conducts particle physics research. It has extensive experimental collaborations with CERN and labs in the United States. SEY experiments were conducted on noble metal surfaces in the materials science INFN-LNF lab. The experimental data shows that SEY is strongly dependent on the chemical state of the material. The results show that the SEY value is different for the surface exposed to atmospheric contaminants as compared to the surface cleaned with $\mathrm{Ar}^{+}$ion sputtering.

The experimental technique consisted of measuring the beam current by positively biasing the Faraday cup in order to prevent backscattered electrons, whereas a negative bias of 75 V was used to measure the target current [32]. All the samples were bombarded with beam energy in the low energy regime, i.e., 0 to $1000 \mathrm{eV}$. Figure 5 shows the comparison of the SEY between the "as received" and atomically cleaned noble metal samples.
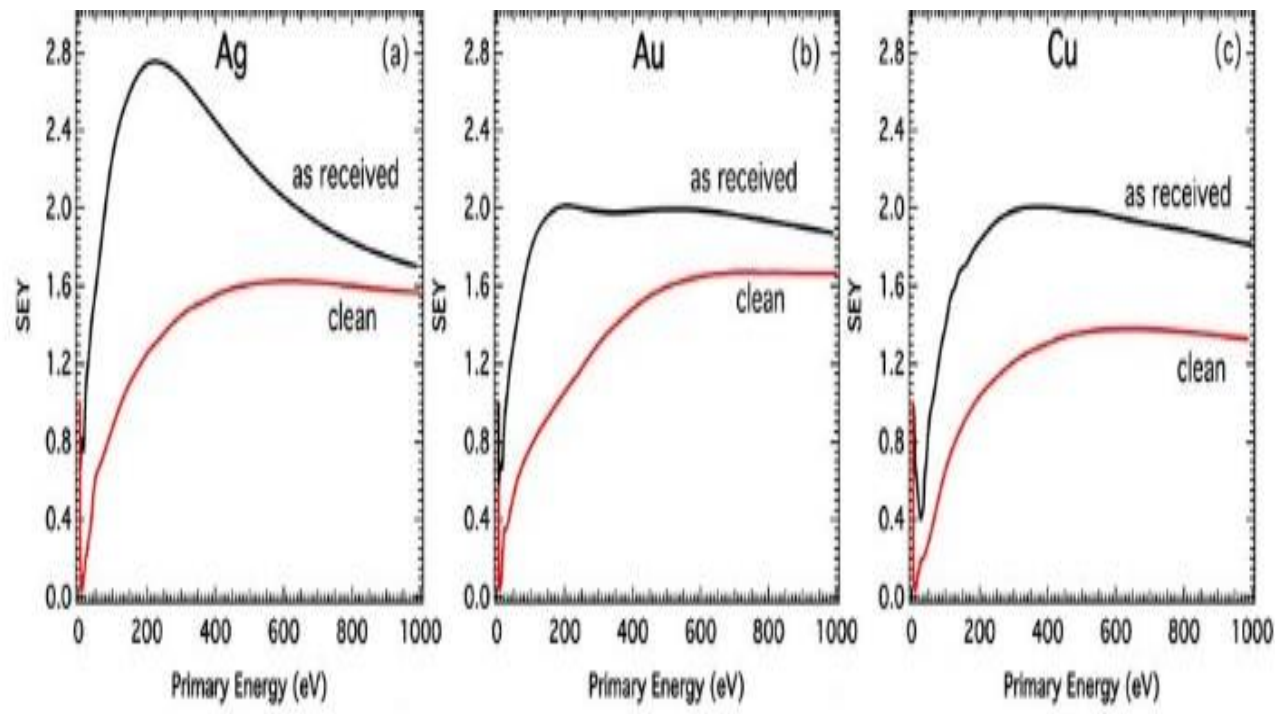

Figure 5: RELATIVE SEY COMPARISON [32]. 
The black curve in Fig. 5 shows the SEY data for the "as received" sample, whereas the red curve shows the SEY for the cleaned sample. It can be clearly seen that SEY is significantly reduced for the cleaned metal surfaces whereas the SEY is higher for the as received samples which were not subjected to any kind of cleaning treatment. It should also be noted from Fig. 5 that, for the clean surface samples, the shape of the SEY curve becomes flatter after around $300 \mathrm{eV}$ beam energy and continues until $1000 \mathrm{eV}$.

These results indicate that the surface chemical state is a key factor in determining the SEY from noble metals [32]. The clean metal surfaces exhibited SEY values that do not exceed 1.6, and even this value was lower for the copper sample, whereas in case of unclean samples, the presence of contaminated layers generated SEY well above 2 [32].

\subsection{DFT-MC-Based Modeling of Secondary Electron Yield}

Density Functional Theory (DFT) is a quantum mechanical tool for electronic structure calculations of materials in order to perform precise materials simulation. DFT based calculations of the material properties can be used to calculate SEY from first principles through Monte Carlo simulation. Therefore, simulations based on DFT calculated properties help with the understanding and analysis of the data acquired in the experiment. They also allow us to understand how structural and electronic properties of materials influence SEY.

The DFT method allows for accurate determination of electronic properties of materials based on the calculation of electron density by solving $n$ one-electron Schrödinger-like equations, which are also known as the Kohn-Sham equations [33]. Some of these properties of interest for the simulation of SEY include (i) density of states, which is the 
number of electronic states that can be occupied by the system at each energy; (ii) Fermi energy, which is the highest occupied energy level of a material at absolute zero temperature; (iii) work function, which is the energy required to withdraw an electron from a material's surface; and more importantly (iv) dielectric properties of the materials, i.e., frequency- and momentum-dependent energy loss function. The energy loss function depends on the frequency $(\omega)$ and momentum transfer $(q)$ (difference in the initial and final electron momenta) and is defined as the imaginary part of the inverse dielectric function as

$$
E L F(q, \omega)=-\operatorname{Im}\left(\frac{1}{\varepsilon(q, \omega)}\right)
$$

where $\varepsilon(q, \omega)$ is the material's frequency- and momentum-dependent dielectric function [34].

The energy loss function is an important quantity as it determines the scattering properties of the solid used in Monte Carlo (MC) simulations (inelastic scattering X-ray spectroscopy and electron energy loss spectroscopy, etc.). More specifically, the frequency- and momentum-dependent energy loss function can be used to calculate inelastic mean free paths, which are then used in MC simulations of secondary electron emission.

\subsection{Monte Carlo Simulation}

The MC method is an important technique in data sciences. It is a statistical computation technique for estimating the value of an unknown quantity. This method of simulation is now extensively applied to material sciences. $\mathrm{MC}$ is used for evaluating many physical quantities necessary for the study of particle-beam interactions with solid targets. A MC scheme considers all the energy losses suffered by each electron in the secondary electron cascade and is used for calculating the secondary electron energy distribution and SEY 
from different materials [35]. MC-based simulation models have now been developed to the point where they can quantitatively predict SEY behavior, given the correct input. These models track the energy loss due to inelastic scattering of the incident electrons and all scattered electrons in the material and the energy changes occurring at the surface during emission. As such, they can be used to calculate the total number of emitted electrons at each energy and angle as a function of the energy and angle of an incident electron. In the DFT-MC approach the data calculated through DFT-based computations, i.e., dielectric properties (or energy loss function), work function, density of states, and Fermi energy are then given as inputs to the MC code to predict SEY for the materials of interest to vacuum electronics [36].

In a nutshell, the combination of DFT and MC can be used for first principles prediction of SEY. As part of the Michigan State University-led AFOSR MURI (Multidisciplinary University Research Initiative) grant, several of the team members are working on simulating SEY curves based on a combination of DFT and MC. The ultimate goal is to assess if the predicted SEY from simulations agrees with the experimental data. In addition, it is useful to understand how structural, electronic, and surface properties of the material influence the generation of the secondary electrons. Figure 6 shows a schematic of SEY obtained through the DFT-MC process. 


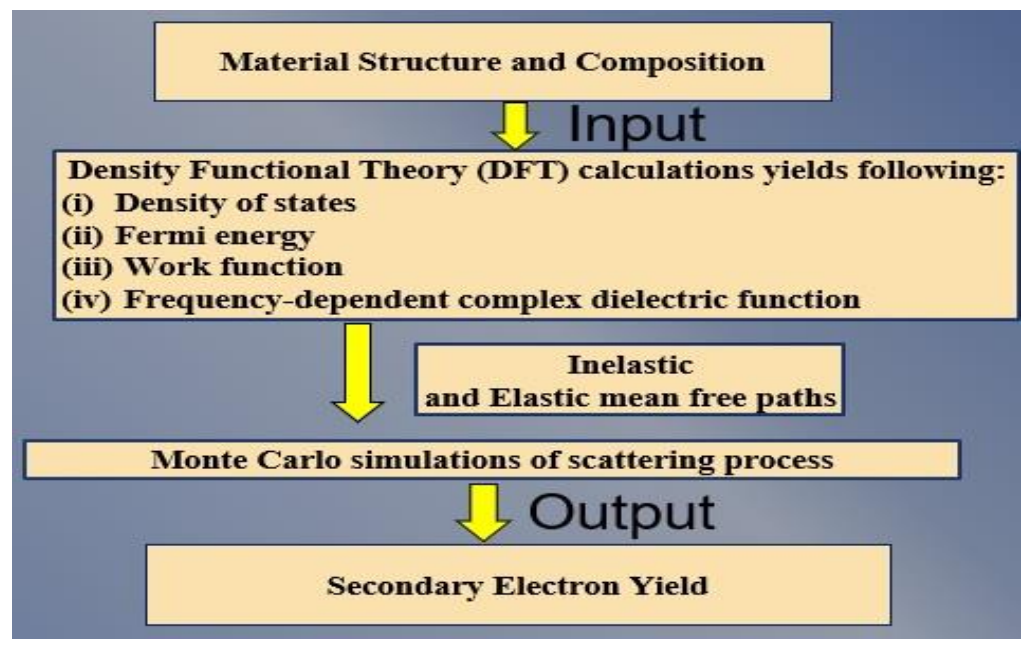

Figure 6: PROCEDURE FOR DETERMINING SEY FROM DFT-MC.

\subsection{Comparison of Experimental Parameters}

Table 2 shows a comparison of different SEY experimental parameters derived from different research papers. Surface treatment methods/cleaning protocols and the bias applied to the sample (while measuring the target sample's current) have a significant role in the measured SEY values. It will be shown in chapter 4 that SEY varies with different surface treatments and applied bias.

Table 2: Comparison of different SEY experimental parameters.

\begin{tabular}{|l|l|c|}
\hline Title of Paper & Surface treatment & Bias Used \\
\hline 1) The SEY of technical materials & 1) Argon Glow Discharge & Not \\
treatment [37]. & (AGD) & mentioned \\
\hline 2) The SEY of noble metal surfaces & 2) Baking & \\
[32]. & & $-75 \mathrm{~V}$ \\
\hline
\end{tabular}




\begin{tabular}{|c|c|c|}
\hline $\begin{array}{l}\text { 3) Research on the SEY of TiZrV-Pd } \\
\text { thin film coatings [38]. }\end{array}$ & $\begin{array}{l}\text { 1) TiZrV and Pd film } \\
\text { coatings. }\end{array}$ & $-40 \mathrm{~V}$ \\
\hline $\begin{array}{l}\text { 4) The SEY from transition metals } \\
\text { [39]. }\end{array}$ & $\begin{array}{l}\text { 1) Electron Conditioning } \\
\text { 2) Baking }\end{array}$ & $-18 \mathrm{~V}$ \\
\hline $\begin{array}{l}\text { 5) SEY Measurements of TiN } \\
\text { Coating and TiZrV Getter Film [40]. }\end{array}$ & $\begin{array}{l}\text { 1) Tin Coating and TiZrV } \\
\text { Film as evident from the } \\
\text { title. }\end{array}$ & $-20 \mathrm{~V}$ \\
\hline $\begin{array}{l}\text { 6) Measuring the effects of Ar-ion } \\
\text { cleaning on the SEY of copper due to } \\
\text { electron impact [41]. }\end{array}$ & $\begin{array}{l}\text { 1) Ar-ion cleaning as evident } \\
\text { from the title. }\end{array}$ & $-20 \mathrm{~V}$ \\
\hline $\begin{array}{l}\text { 7) Secondary Electron Emission from } \\
\text { Plasma-Generated Nanostructured } \\
\text { Tungsten Fuzz [42]. }\end{array}$ & $\begin{array}{l}\text { 1)Plasma-Generated } \\
\text { Nanostructured Tungsten as } \\
\text { evident from the title. }\end{array}$ & $-20 \mathrm{~V}$ \\
\hline $\begin{array}{l}\text { 8) Influence of air exposures and } \\
\text { thermal treatments on the SEY of } \\
\text { copper [43]. }\end{array}$ & $\begin{array}{l}\text { 1) Ar-ion sputtering } \\
\text { 2) Baking }\end{array}$ & $\begin{array}{c}\text { Not } \\
\text { mentioned }\end{array}$ \\
\hline $\begin{array}{l}\text { 9) Electron Conditioning of Technical } \\
\text { Aluminum Surfaces: Effect on the } \\
\text { SEY [44]. }\end{array}$ & 1) Electron Conditioning & $-20 \mathrm{~V}$ \\
\hline $\begin{array}{l}\text { 10) Empirical modeling and MC } \\
\text { simulations of SEY suppression of } \\
\text { laser-drilled micro-porous gold } \\
\text { surfaces [45]. }\end{array}$ & $\begin{array}{l}\text { 1) Laser drilled micro- } \\
\text { porous gold surfaces. }\end{array}$ & $0 \mathrm{~V}$ \\
\hline
\end{tabular}




\subsection{Scope and Organization of Thesis}

The motivation for this thesis is to find materials with low SEY for application in RF and microwave communication devices. The materials list was suggested by industrial aerospace partners on the MURI team. The primary objective of this experimental research is to investigate materials and different cleaning protocols, especially focusing on ultrasonic cleaning treatment of the material surfaces that could lower SEY. In addition, the effect of in-situ baking on reducing the SEY has also been studied. The "Dose Effect" studied by Prashanth Kumar [21] has also been investigated for some of the materials. It is shown that SEY is a surface sensitive phenomenon and electron emission depends on how surface of the material is being prepared.

The remainder of this thesis is divided into following chapters: Chapter 2 briefly covers the experimental set up and measurement methods applied to determine SEY. Chapter 3 describes ex-situ and in-situ sample cleaning procedures adopted in this study. Chapter 4 presents experimental results for different materials and relative SEY comparison between different cleaning protocols employed for surface cleaning. Finally, chapter 5 presents conclusions and recommendations for future work. 


\section{Chapter 2}

\section{Experimental Set-Up}

\subsection{Vacuum System}

A vacuum system is critical in a SEY experimental setup since experiments require ultrahigh vacuum (UHV) conditions. A typical vacuum system consists of a vacuum chamber, combination of pumps, valves, and pipes which establish a region of low pressure [46]. The vacuum chamber used in this work is made of stainless steel and has a cylindrical body which is approximately 7 liters in volume. It has multiple 2.75 inch Conflat flange (CF) ports which are used for feedthroughs for various applications, such as heating the sample, measuring the temperature of a mounted sample via a thermocouple device, sample biasing through a BNC connector, and for installing instruments such as a Residual Gas Analyzer (RGA). One 8-inch diameter $\mathrm{CF}$ window is used as a viewing port and for installing and uninstalling the sample inside the chamber. Two 6 inch ports are used to connect two turbo pumps.

The electron gun and the sample holder are housed inside the vacuum chamber. The linear and angular manipulators are mounted on the vacuum chamber. A linear manipulator is used to linearly change the position of sample inside the chamber and an angular manipulator is used to change the angle of incidence of the electron beam on the sample. There is an angular scale on the angular manipulator to give the angle of incidence of the 
electron beam. Figure 7 shows a photo of the experimental setup with a number of important components labeled.

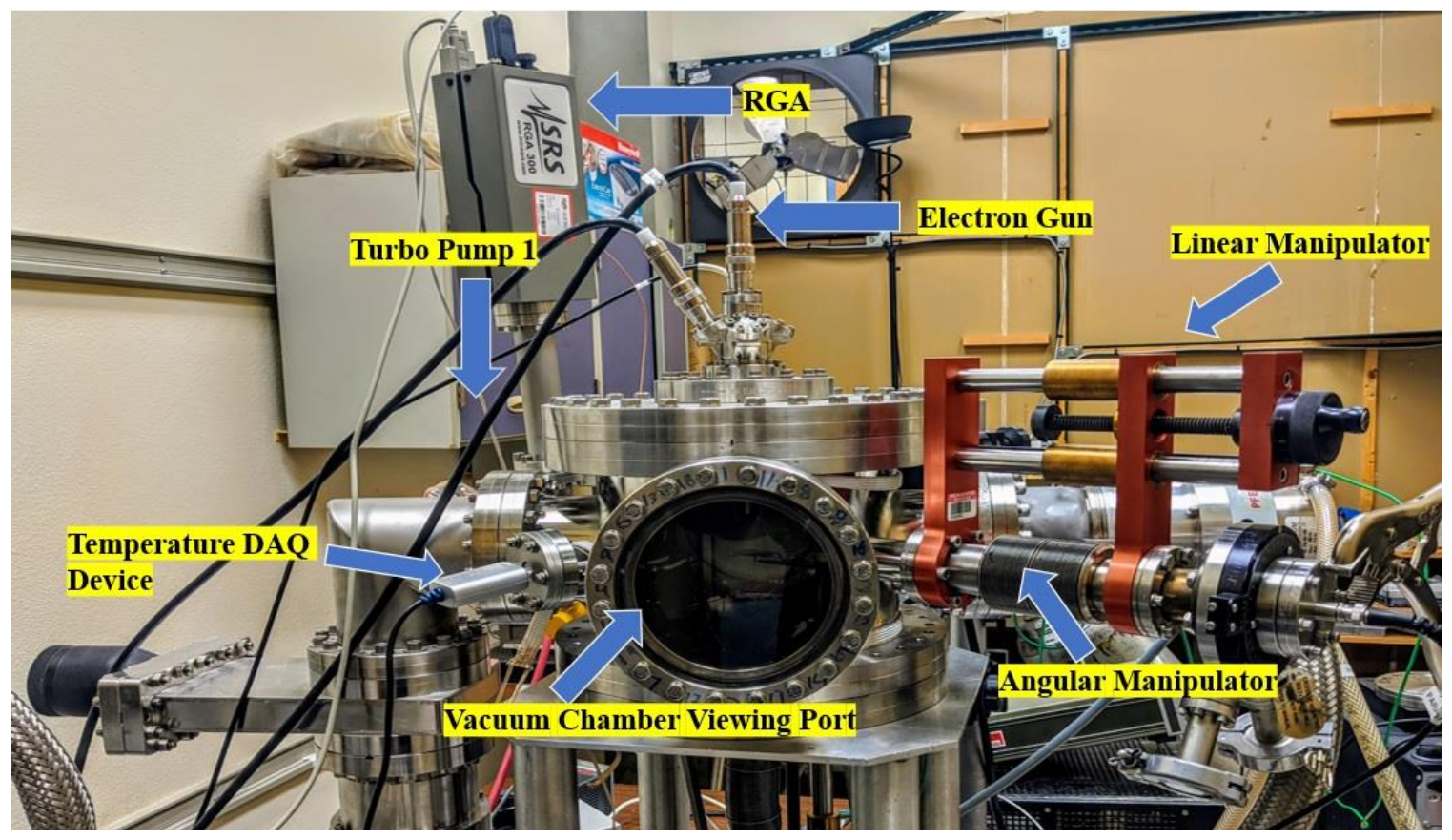

Figure 7: PHOTOGRAPH OF THE EXPERIMENTAL SETUP.

\subsubsection{Establishing Ultra-High Vacuum}

The first step in the experimental procedure involves establishing a vacuum inside the stainless-steel vacuum chamber. A vacuum base pressure of around $10^{-8}$ Torr was achieved by using the combination of one roughing and two turbo molecular pumps. A summary of the technical specifications of the pumps relevant to the experiment is shown in Fig. 8. A flanged Bayard - Alpert uncoated glass tube (hot iridium filament ion gauge) with 1.0" diameter Kovar Port (DUNIWAY part \# I-100-K) and Terranova model type 934-UHV wide - range vacuum controller were used to monitor the pressure below $10^{-3}$ Torr. 


\begin{tabular}{|c|c|c|c|}
\hline Operational Pumps & $\begin{array}{l}\text { Roughing / Dry } \\
\text { Scrolling Pump }\end{array}$ & Turbo Pump l & Turbo Pump 2 \\
\hline Manufacturer & $\begin{array}{l}\text { Varian Vacuum } \\
\text { Technologies }\end{array}$ & PFEIFFER Vacuum & PFEIFFER Vacuum \\
\hline Model & $\begin{array}{c}\text { TriScroll1 } 1{ }^{\mathrm{MM}} 300 \\
\text { Series }\end{array}$ & TMU 260C & TPH 190 \\
\hline $\begin{array}{l}\text { Peak Pumping } \\
\text { Speed } \\
\text { (Liters/Second) }\end{array}$ & 4.16 (at $60 \mathrm{~Hz}$ ) & $210\left(\mathrm{~N}_{2}\right)$ & $190\left(\mathrm{~N}_{2}\right)$ \\
\hline Cooling System & Air cooled & Water cooled & Water cooled \\
\hline Controller & N/A & TCP 380 & TCP 300 \\
\hline
\end{tabular}

Figure 8: PUMP SPECIFICATIONS [47] [48] [49].

A roughing pump is used to bring the pressure from atmospheric level to $10^{-2}$ Torr and then a combination of two turbomolecular pumps are used to further bring the pressure to the level of $10^{-8}$ Torr. The roughing pump is connected to the output of two turbomolecular pumps. A cooling system for the turbo pumps consists of a chiller which regulates the temperature of a distilled water at $15^{\circ} \mathrm{C}$.

Conflat copper gaskets are used for ensuring high vacuum seals. The time to achieve UHV inside the chamber depends on the exposure time of the chamber to air. Water vapor present in the air is adsorbed onto the walls of the vacuum chamber which leads to increase in pumpdown time.

\subsubsection{Importance of Ultra-High Vacuum}

The creation of UHV inside the chamber is necessary to study the SEY of materials which are to be used in fabrication of VEDs in space RF technologies. It is always of interest to keep the sample under test as clean as possible from contaminations. At atmospheric 
pressure surfaces are constantly bombarded by molecules which can either bounce from the surface, attach themselves to the surface, or perhaps chemically react with a surface [19]. The presence of UHV conditions will ensure that the rate by which the molecules hit the surface of sample will be considerably reduced and hence help keep the surface clean. The base pressure for the experiment was maintained around $3 \times 10^{-8}-5 \times 10^{-8}$ Torr except while heating the sample, when the pressure increased to $1 \times 10^{-7}$ Torr.

UHV is relevant to SEY experiments in the following ways.

(1) Monolayer Formation Time:

One of the important motivations for establishing UHV is that it increases monolayer formation time which minimizes the formation of monolayers of contaminants on the surface of samples installed inside the chamber. The time to saturate a surface with one layer of molecules is a function of the molecular arrival rate, $\Gamma$, and the size of a molecule. Assuming each molecule sticks and occupies surface area $\mathrm{do}^{2}$, the time to form a monolayer is given by

$$
\mathrm{t}_{\mathrm{ml}}=\frac{1}{\Gamma \mathrm{do}^{2}}=\frac{4}{\mathrm{n} * \mathrm{v} * \mathrm{do}^{2}}
$$

In Eq. (2.1) tml is the time to form a monolayer where

$\Gamma=$ Flux of an ideal gas striking a unit surface or crossing an imaginary plane of unit area from one side in particles $/ \mathrm{m}^{2} \mathrm{~s}=\mathrm{nv} / 4$

$\mathrm{n}=$ particle density in $\mathrm{m}^{-3}$

$\mathrm{v}=$ average velocity in $\mathrm{ms}^{-1}$. 
At room temperature, a monolayer of air will form in about $2.5 \mathrm{~s}$ at a pressure of $3 \times 10^{-6}$ Torr. The formation time will be longer if the sticking coefficient is less than unity.

\section{(2) Mean Free Path}

The mean free path is the distance travelled by a randomly distributed molecule in a gas before colliding with another [46]. The average mean free path, $\lambda$, is based on kinetic theory according to

$$
\lambda=\frac{1}{2 \frac{1}{2} * \pi * \mathrm{do}^{2} * n}
$$

where

do $=$ molecular dimeter in meters

$\mathrm{n}=$ gas density in molecules $/ \mathrm{m}^{3}$. The above equation clearly shows that the mean free path is gas density dependent, and therefore, also pressure dependent. A large mean free path is desired to ensure that minimum interaction takes place between the incident electron beam and air molecules.

At room temperature, the mean free path is given by,

$$
\lambda(\mathrm{mm})=\frac{6.6}{\mathrm{p}}
$$

where $\mathrm{P}$ is pressure in Pascals whereas 1 pascal $=0.007$ Torr.

$$
\lambda(\mathrm{mm})=\frac{0.05}{\text { pressure in Torr }}
$$

The base pressure for this experiment is around $5^{*} 10^{-8}$ Torr which yields a mean free path $\approx 1 \mathrm{~km}$ according to Eq. (2.4). 
Kinetic theory describes the distribution of free path as [19]

$$
\mathrm{N}=\mathrm{N}^{\prime} e^{\frac{-x}{\lambda}}
$$

where $\mathrm{N}^{\prime}$ is the number of molecules in the volume and $\mathrm{N}$ is the number of molecules that travel a distance before colliding with another molecule. Equation (2.5) states that $63 \%$ of the collision occurs in a distance $0 \leq x \leq \lambda$, while $37 \%$ of the collision occurs in the range $0 \leq x \leq 5 \lambda$. Only about $0.6 \%$ of the particles travel distances greater than $5 \lambda$ without suffering any collision.

\subsubsection{Pressure vs. time curve}

The pump down time from atmospheric pressure depends on exposure time of the chamber to atmosphere and the contaminants adsorbed into pores of stainless steel chamber. Contaminants consist mainly of adsorbed atoms and molecules of atmospheric and other (contaminant) gases. Consequently, it is important to have as good a vacuum as possible. The pump down time to the $10^{-8}$ Torr range varies from 30 to 90 hours depending upon the factors already mentioned.

Figure 9 shows the pressure vs. time curve for the case when the chamber was exposed to atmospheric pressure for a long time. The pressure vs. time curve is plotted in Lab View. Figure 9 shows that initially pressure decays exponentially down to the level of around $10^{-6}$ Torr and then continuous spikes are observed at further lower pressure. 


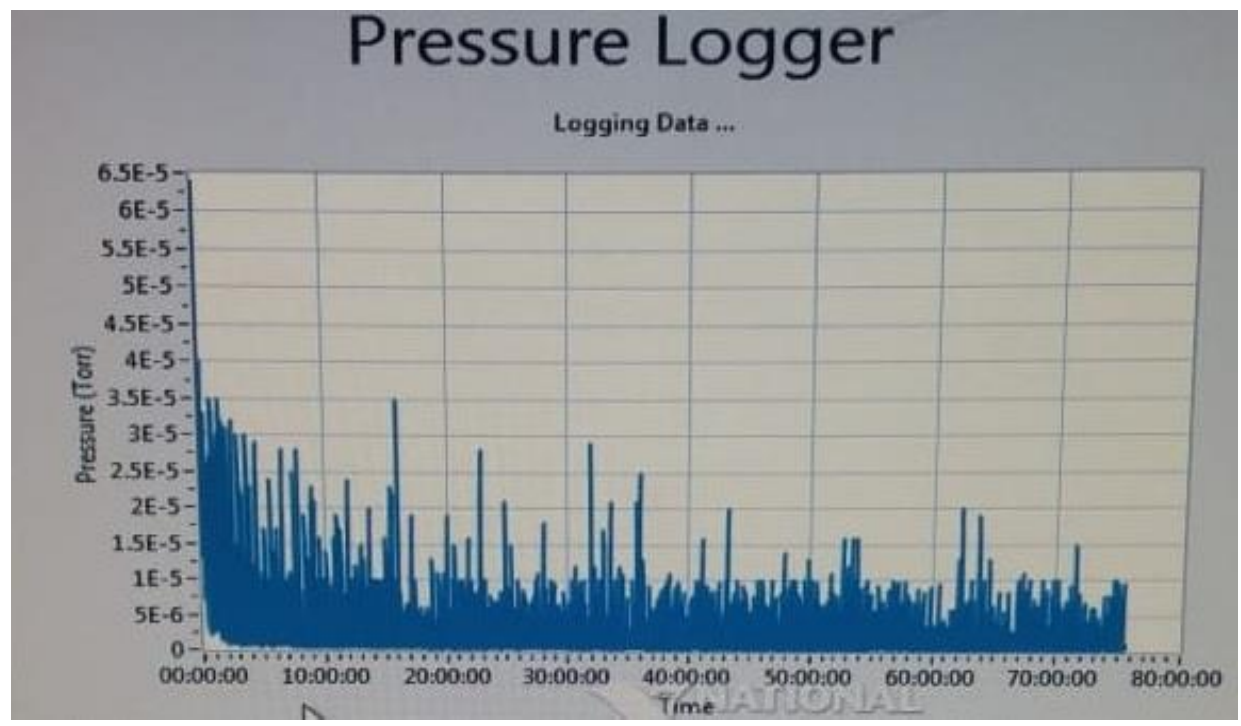

Figure 9: PRESSURE VS. TIME CURVE AS MEASURED BY ION GAUGE.

The vertical axis in Fig. 9 shows the pressure inside the chamber in Torr and the horizontal axis shows the time in hours. Due to the long exposure time, the pressure is widely varying and is around $10^{-6}$ Torr even after 75 hours have passed since the pumps were turned on. Figure 10 shows the pressure vs. time curve for the case when exposure time of chamber was kept short. The estimated short and long exposure times of the vacuum chamber to atmospheric level indicate a time duration of around 3-4 hours and 18-24 hours, respectively.

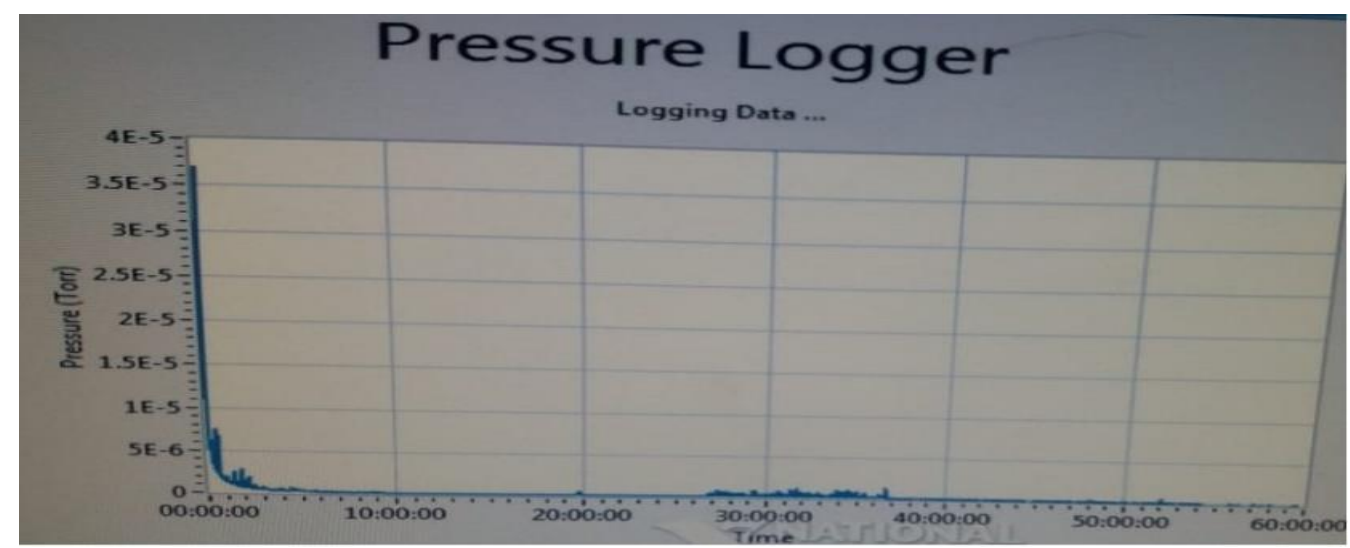

Figure 10: PRESSURE VS. TIME (HOURS) CURVE. 


\subsection{Schematic of the Experiment}

A simplified schematic of the experiment is shown in Fig.11. The electron beam impinges normally on the surface of the sample, which is electrically isolated from ground except through the current meter as shown. As described in chapter 1, the SEY is measured using the ratio of beam and target current, i.e., $\delta=1-\mathrm{I}_{t} / \mathrm{I}_{\mathrm{b}}$. A battery was used to bias the sample with either positive or negative voltage with respect to the grounded vacuum chamber wall in order to measure the primary beam and target currents, respectively. Because conductive metal surfaces were used in these experiments, a positive $(+100 \mathrm{~V})$ with respect to ground bias was applied to the sample to measure primary beam current (Ip) since all of the secondary electrons could be recaptured by the surface of the sample.

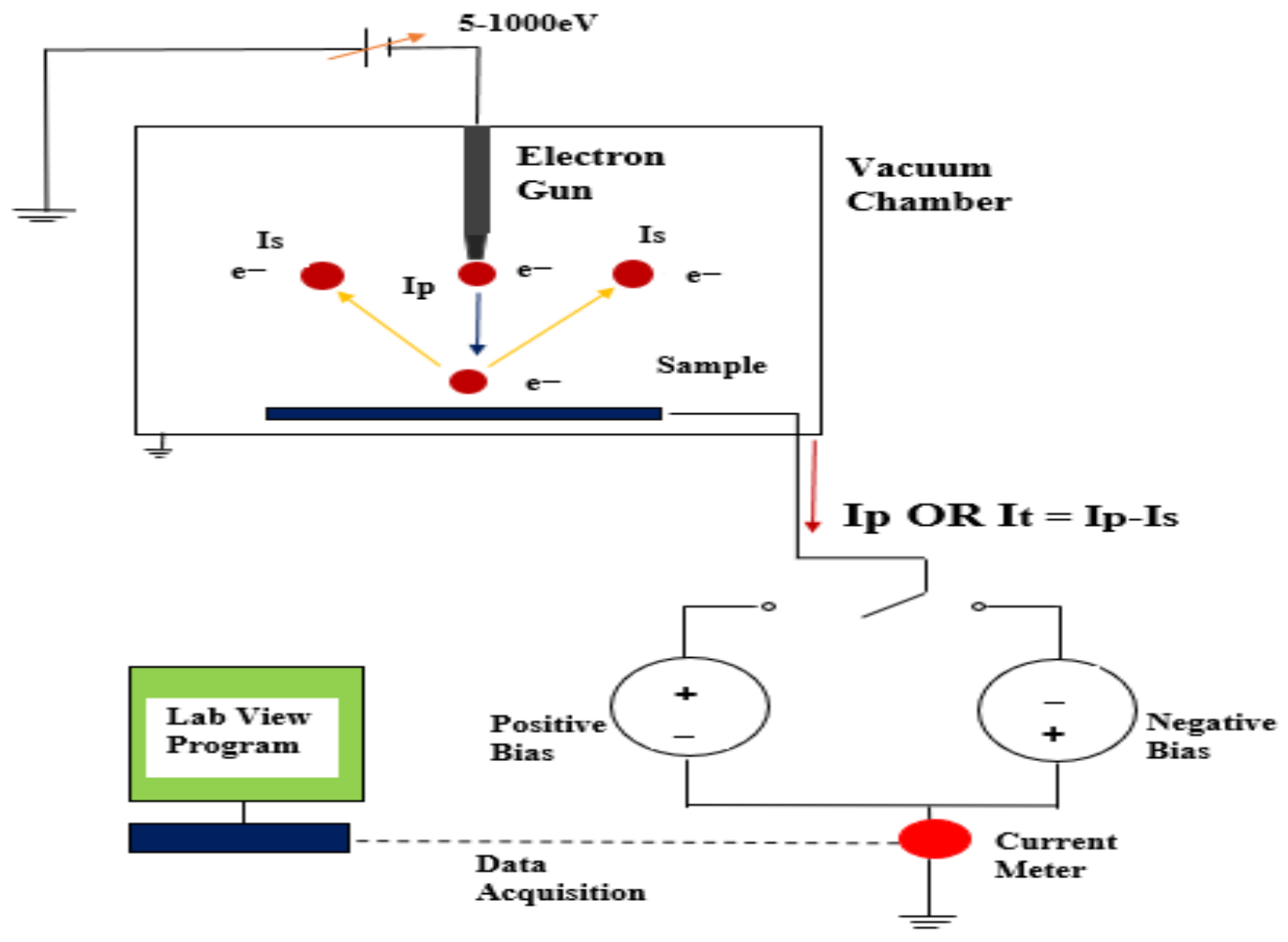

Figure 11: SEY EXPERIMENT SCHEMATIC. 
In order to determine SEY, a negative bias of $-20 \mathrm{~V}$ is applied to the sample while measuring the target current (It) so that all of the secondary electrons are repelled from the sample surface (e.g., there is no space charge buildup near the sample surface). A mechanical relay is used to switch between positive and negative voltages. Batteries were utilized in order to eliminate unwanted current paths to ground that might be present with power supplies. An electrometer (Keithley model 6514) with a capability of measuring current in the range (100 aA-21 mA) was used to measure currents (beam and target). A Lab View program controls the electron gun, electrometer, and bias relay. A GPIB (General purpose Interface Bus) cable is used to transfer information from electrometer to the NI (National Instruments) card in the computer.

Some of the detail of components involved in the experiment are given below.

\subsubsection{Electron Gun}

For this experiment, a low energy ELG-2 electron gun from Kimball Physics was used to bombard the samples with an electron beam. The electron gun has the capability to generate electrons with beam energy varying from $5 \mathrm{eV}$ to $1000 \mathrm{eV}$, or to $2000 \mathrm{eV}$ with the high energy option. The Kimball Physics EGPS-2 power supply was used to control gun parameters, e.g., beam energy, anode voltage, and focus voltage. The power supply for the electron gun was controlled either manually or remotely through a Lab View program. Manual control was accomplished by turning the potentiometers on the power supply. Two 68-pin data acquisition cables were used to connect the lab computer to the power supply for remote control. Data acquisition boards in the computer were used to monitor and record all data through Lab View from the power supply and Keithley current meter.

Some of the operating parameters of the electron gun are mentioned in Table 3 [50]. 
Table 3: Specifications of the electron gun.

\begin{tabular}{|l|l|}
\hline Beam Energy & $\begin{array}{l}\text { Standard : } 5 \mathrm{eV} \text { to } 1000 \mathrm{eV} \\
\text { High Energy option: } 50 \mathrm{eV} \text { to } 2 \mathrm{keV}\end{array}$ \\
\hline Spot Size & $0.5 \mathrm{~mm}$ to $5 \mathrm{~mm}$ \\
\hline Cathode & $\begin{array}{l}\text { Standard: Refractory metal } \\
\text { Optional: Low-light barium oxide } \\
\text { coated and Thoria coated iridium }\end{array}$ \\
\hline Operating Pressure & $10^{-11}-10^{-5} \mathrm{Torr}$ \\
\hline Working Distance & Variable: $5 \mathrm{~mm}$ to $100 \mathrm{~mm}$ \\
\hline Beam Current & $10 \mathrm{nA}-10 \mu \mathrm{A}$ \\
\hline Bakeable Temperature & $350 \mathrm{C}$ \\
\hline
\end{tabular}

The power supply for the electron gun was remotely controlled through a Lab View program provided by the Kimball Physics and was optimized according to requirements of experiment.

The power supply of the electron gun allows two types of remote access:

(1) Remote monitoring of all meters

(2) Remote programming of all individual power supplies. 
Remote programming allowed for controlling individual control parameters of the gun. The control parameters were beam energy, emission current, first anode voltage and grid voltage.

Data acquisition boards in the computer were used to monitor and record all data through a Lab View program from the power supply and Keithley electrometer. All current measurements and control parameters of the electron gun were saved by Lab View as an excel file.

\subsubsection{Sample Holder}

A highly conductive path was made for the flow of beam and target currents. To ensure this, samples for the experiment were mounted on the copper mounts. Ceramic screws were used to isolate the sample from ground i.e. brass extension and chamber. The copper mount was elevated so that the desired distance between electron gun and the sample could be achieved. All materials used in the construction of sample holder were capable of enduring temperature up to $300-350^{\circ} \mathrm{C}$ for baking.

\subsubsection{Keithley Electrometer [51]}

The Keithley electrometer represented as the current meter in the schematic as shown in Fig. 11 is capable of measuring currents from $100 \mathrm{aA}-21 \mathrm{~mA}$ and is suited for this experiment. The current in this experiment was in the range of nanoamperes to around 0.5

$\sim 1 \mu \mathrm{A}$. The meter is used in the Amps mode and set to auto range. Initialization (zero check, zero correct and range) was automated and the meter warmed up for approximately an hour for accurate measurements as recommended in the manual. 
The range of current measurements and accuracy with each range is shown in Table 4. below.

Table 4: Current range of Keithley electrometer.

\begin{tabular}{|c|c|c|c|}
\hline AMPS & $\begin{array}{c}\text { 51/2 DIGIT } \\
\text { RESOLUTION }\end{array}$ & $\begin{array}{c}\text { ACCURACY } \\
\text { (1 Year) } 1 \\
18^{\circ}-28^{\circ} \mathrm{C} \\
\pm(\% \text { rdg+counts })\end{array}$ & $\begin{array}{c}\text { TEMPERATURE } \\
\text { COEFFICIENT } \\
0^{\circ}-18^{\circ} \mathrm{C} \& 28^{\circ}-50^{\circ} \mathrm{C} \\
\pm(\% \text { ordg+counts }) /{ }^{\circ} \mathrm{C}\end{array}$ \\
\hline $20 \mathrm{pA}$ & $100 \mathrm{aA}^{2}$ & $1+30$ & $0.1+5$ \\
\hline $200 \mathrm{pA}$ & $1 \mathrm{fA}^{2}$ & $1+5$ & $0.1+1$ \\
\hline $2 \mathrm{nA}$ & $10 \mathrm{fA}$ & $0.2+30$ & $0.1+2$ \\
\hline $20 \mathrm{nA}$ & $100 \mathrm{fA}$ & $0.2+5$ & $0.03+1$ \\
\hline $200 \mathrm{nA}$ & $1 \mathrm{pA}$ & $0.2+5$ & $0.03+1$ \\
\hline $2 \mu \mathrm{A}$ & $10 \mathrm{pA}$ & $0.1+10$ & $0.005+2$ \\
\hline $20 \mu \mathrm{A}$ & $100 \mathrm{pA}$ & $0.1+5$ & $0.005+1$ \\
\hline $200 \mu \mathrm{A}$ & $\ln \mathrm{A}$ & $0.1+5$ & $0.005+1$ \\
\hline $2 \mathrm{~mA}$ & $10 \mathrm{nA}$ & $0.1+10$ & $0.008+2$ \\
\hline $20 \mathrm{~mA}$ & $100 \mathrm{nA}$ & $0.1+5$ & $0.008+1$ \\
\hline
\end{tabular}

The electrometer was interfaced with Lab View through an IEEE 488 - GPIB cable. The Lab View program enabled display, measurement and recording of beam and target current data in real time. The real time measurement of data was important as it helped with correcting any fluctuations observed while saving data.

\subsubsection{Relay Circuit Details}

Two IDEC (RY2KS), electromechanical, gold plated contact latching relays were used [52] for switching the battery bias. The reason for choosing this specific relay was driven by the concern that a solid state relay might drain small amounts of current away from the electrometer. The latching maintains its set or reset condition until it receives the next inverting input even if the voltage is interrupted. Gold plated contacts were necessary due to the low magnitude currents involved. The relay trigger circuit was designed to switch to 
positive and negative of voltage in order to measure beam and target current respectively. A schematic of the relay and trigger circuit is shown in Fig. 12 below.

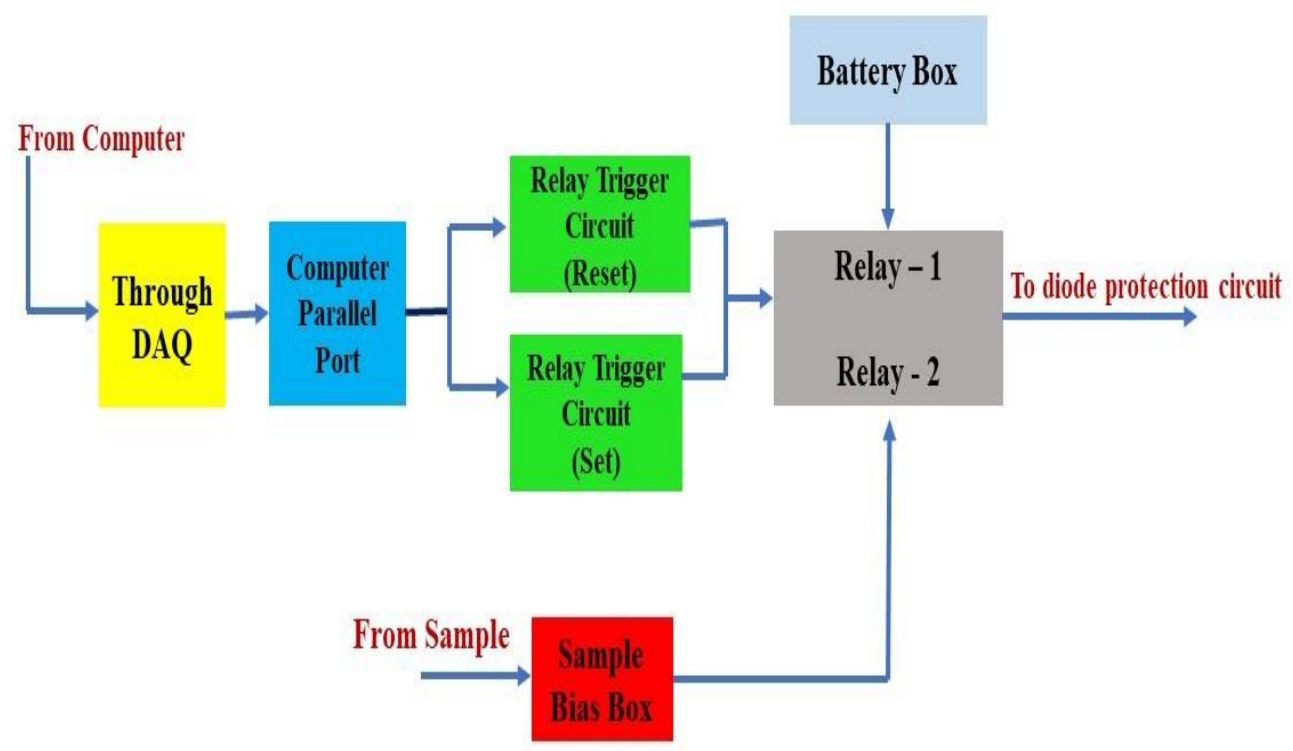

Figure 12: SCHEMATIC OF RELAY AND TRIGGER CIRCUIT USED FOR SWITCHING BATTERY BIAS.

The DAQ device is controlled through its driver installed on the computer. Channel 0 and channel 1 were configured to send command of appropriate voltage, i.e., $5 \mathrm{~V}$ to the input of the relay through the computer's parallel port. The choice of parallel port was made keeping in view the simpler design and ease in software programming as compared to that of a serial port.

The output current from a parallel port is not enough for driving the relay circuit. Therefore, a relay driver circuit was needed to amplify the current [53]. The relay required a current of $>100 \mathrm{~mA}$ and voltage $>5 \mathrm{~V}$ DC. In order to achieve current of order of higher than 100 mA, a transistor switch circuit was used to amplify the current signal. An LTspice® simulation of a transistor switch circuit used to achieve a desired goal is shown in Fig. 13. 


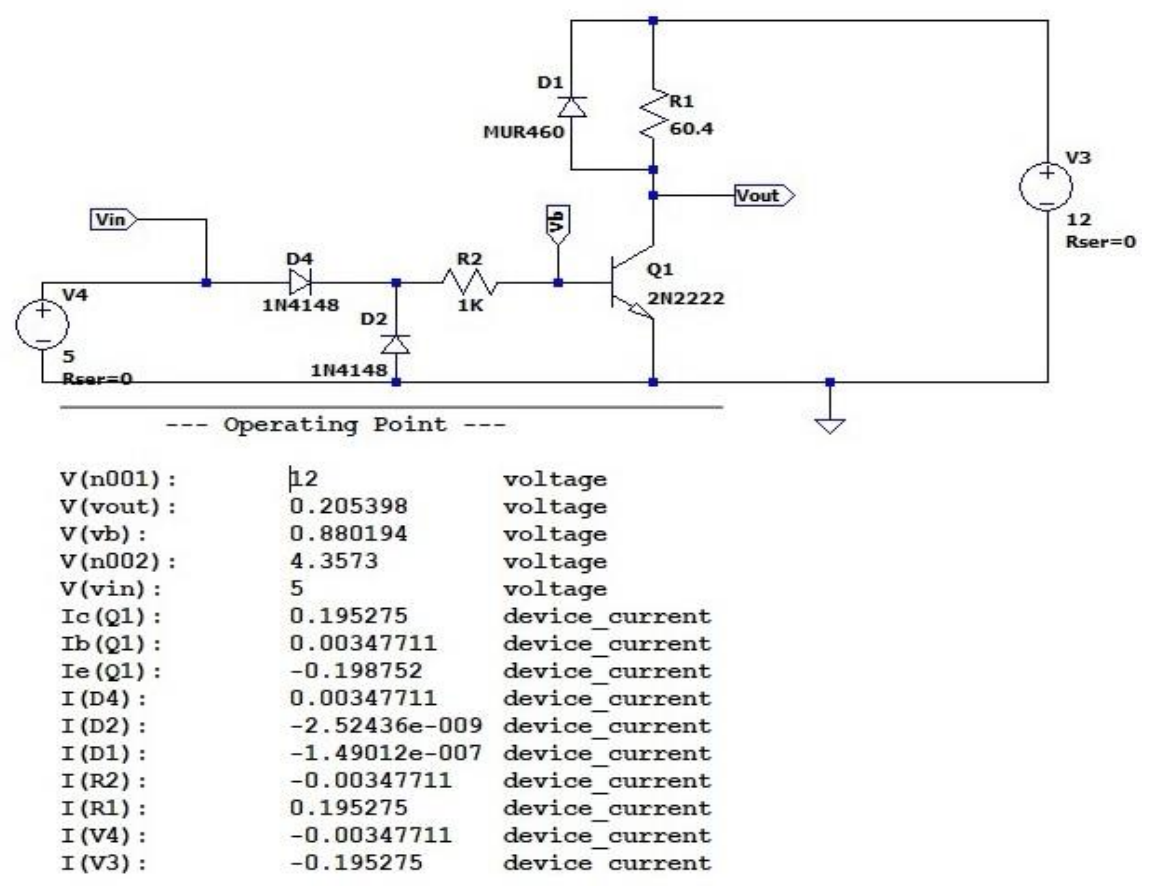

Figure 13: LTSPICE SIMULATION OF RELAY AND TRIGGER CIRCUIT.

A bias of $12 \mathrm{~V}$ was used to bias the transistor collector to ensure triggering of relay. Due to the large amount of inductance associated with relays, a large voltage spike can be generated during switching. The MUR460 diode in parallel with the relay is used to protect from high voltage inductive kickback from the relay coil. Two 1 N4148 diodes protect the parallel port against voltages greater than $+5 \mathrm{~V}$ signals and also against reverse polarity signals. The combination of two such circuits, as shown in Fig. 13, were used for set and reset operations of the relay. These circuits share a common ground with the computer parallel port.

\subsubsection{Battery Box}

The battery box housed an assembly of $1.5 \mathrm{~V}, 9 \mathrm{~V}$, and $45 \mathrm{~V}$ batteries connected in series. Each of the batteries was further connected to a rotary switch that allows selection of the range of voltages. The design of the batteries connected in series provided flexibility in 
choosing a combination of voltages between $1.5 \mathrm{~V}$ to around $200 \mathrm{~V}$. The number of batteries was chosen in such a way that the total sum of the voltages in each series is greater than the minimum voltage of the following series, i.e. $1.5 * 7=10.5 \mathrm{~V}>9 \mathrm{~V} * 6=54 \mathrm{~V}$ and $45^{*} 3=135 \mathrm{~V}$. The total sum of voltages resulting from three sets of series batteries $\approx 200$ V.

The simplified schematic of the bias circuit is shown in Fig. 14.

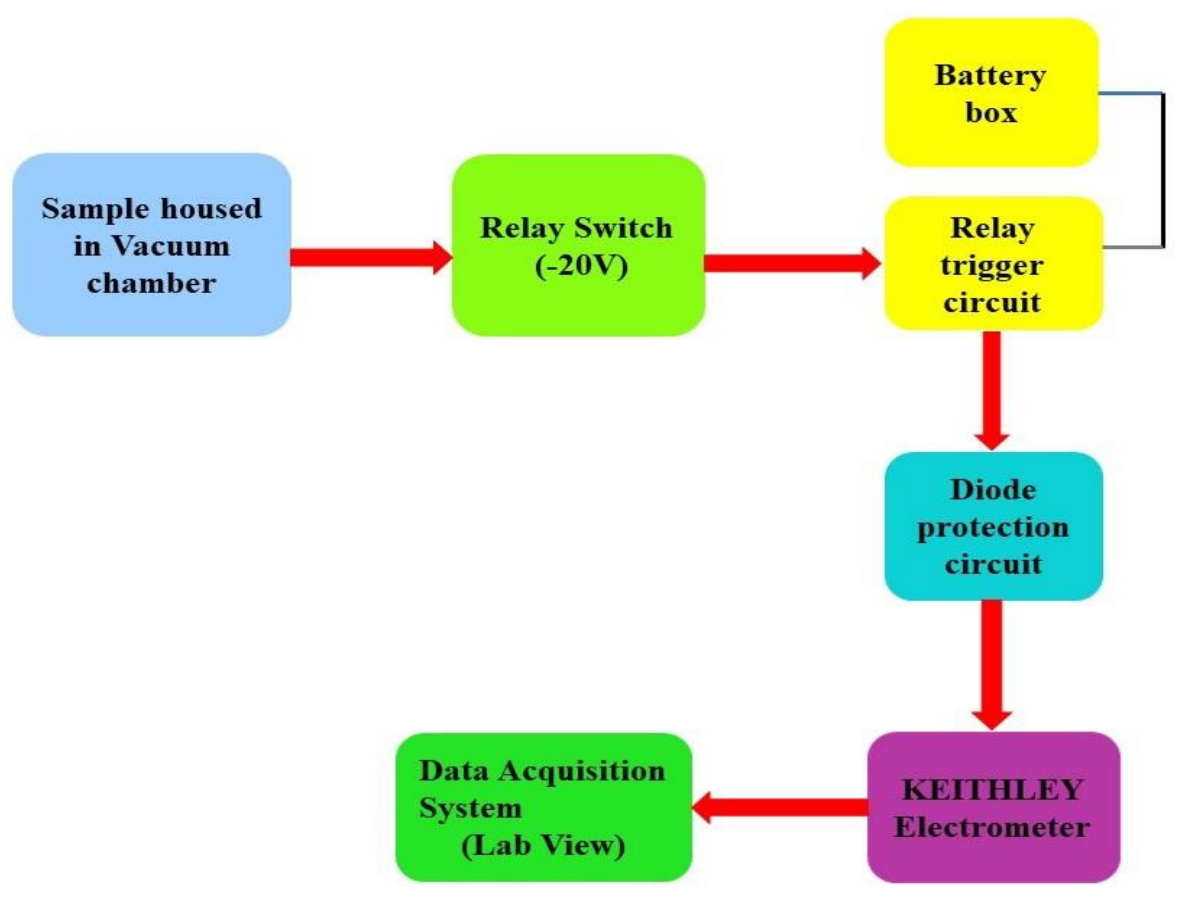

Figure 14: BLOCK DIAGRAM OF THE BATTERY BIAS CIRCUIT.

The sample in the vacuum chamber was connected to the battery box through a relay and trigger circuit. The relay and trigger circuit were used for making beam current (positive bias) and target current (negative bias) measurements, respectively. The diode protection circuit was used to protect electrometer from high currents in case too much current is sourced by the battery bank. The data from the electrometer was then transmitted to the computer for real-time display. 


\subsubsection{Labview Program}

A Lab View program was used for data acquisition and tailored according to the features required to run the experiment and diagnostics. The original Lab View program offered by Kimball Physics was included as a subroutine. The electron gun and power supply were controlled using two 68-pin data acquisition cables. A General GPIB cable was used to feed current data from the electrometer to the NI card installed in the computer.

The Lab View program allowed setting beam energy, focus, and first anode voltage as the variables of the electron gun. The emission current of the electron gun was set manually to the desired value by slowly increasing the source voltage in steps. SEY was measured as a function of beam energy. The program permits changing step size and the time difference between steps while scanning beam energy during SEY measurements.

The electrometer was then initialized and the gun was warmed up for a 30-minutes. Data acquired was saved in Excel file format. Emission current was turned off once the experiment was completed, followed by the decrement in beam energy and first anode voltage to ensure the safe shutdown of the electron gun.

\subsection{Residual Gas Analyzer (RGA)}

The Stanford Research Systems (SRS) RGA 300 consisting of a quadrupole probe was used to determine the residual gases in the vacuum chamber and by-products formed from the bombardment of electrons on the sample surface. The RGA works by separating and detecting ions produced by the primary electron and target interaction according to their charge to mass ratio. Data plots were acquired through the software provided by the vendor through CD [54]. 


\subsection{Heater and Temperature Measurements}

Baking constitutes an important part of this experiment. A quartz lamp heater from Kurt J. Lesker company model \#QLH0500 placed directly under the sample as shown in Fig. 15 was used to bake the sample. The quartz heater worked based on triple heating effects by producing infrared, visible, and ultraviolet radiation. This triple heating effect ensured that contaminates adhered to the surface of the sample through oxidation or adsorption [19] were removed.

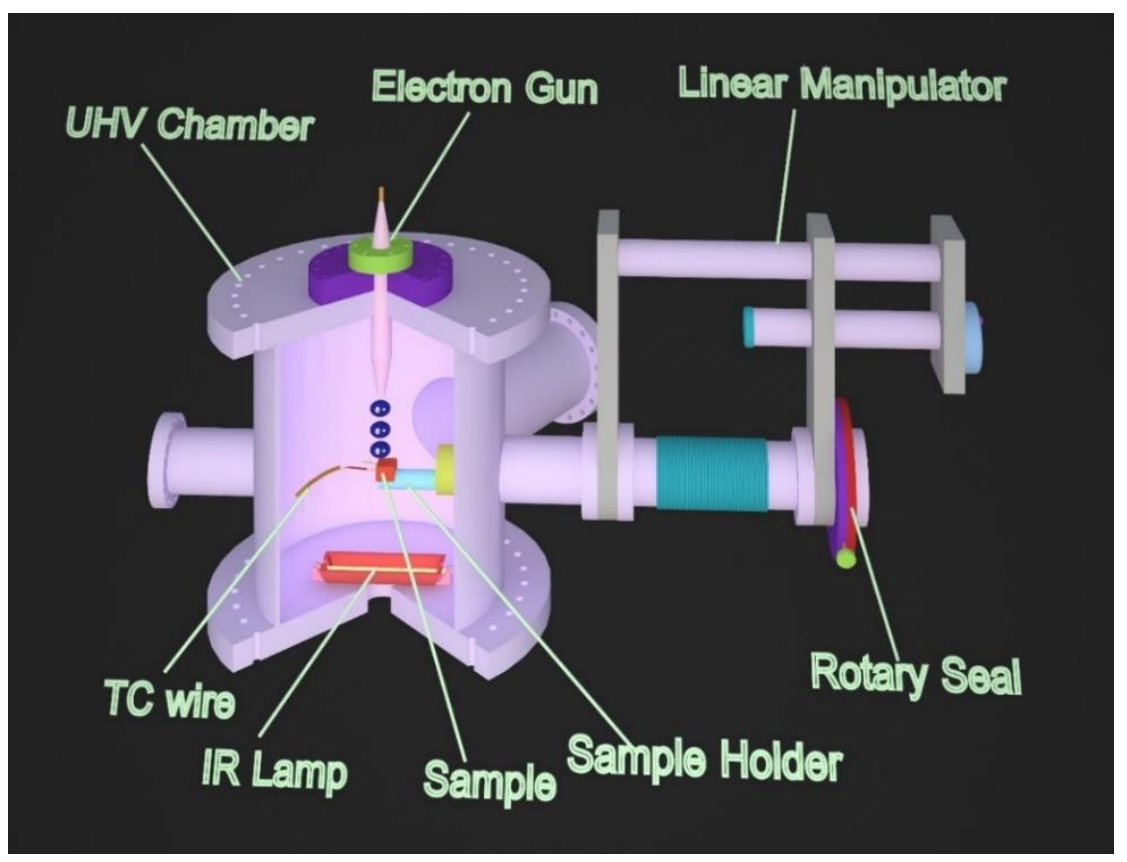

Figure 15: 3D VIEW OF THE CHAMBER INCLUDING THE HEATER USED FOR SAMPLE BAKING.

The single tungsten filament bulb with a rating of $120 \mathrm{~V}, 500 \mathrm{~W}$ was mounted in an electropolished stainless steel ' $\mathrm{V}$ ' reflector so that energy radiated from the bulb could be focused directly on the material surface. The heater lamp assembly is shown in Fig. 16. 


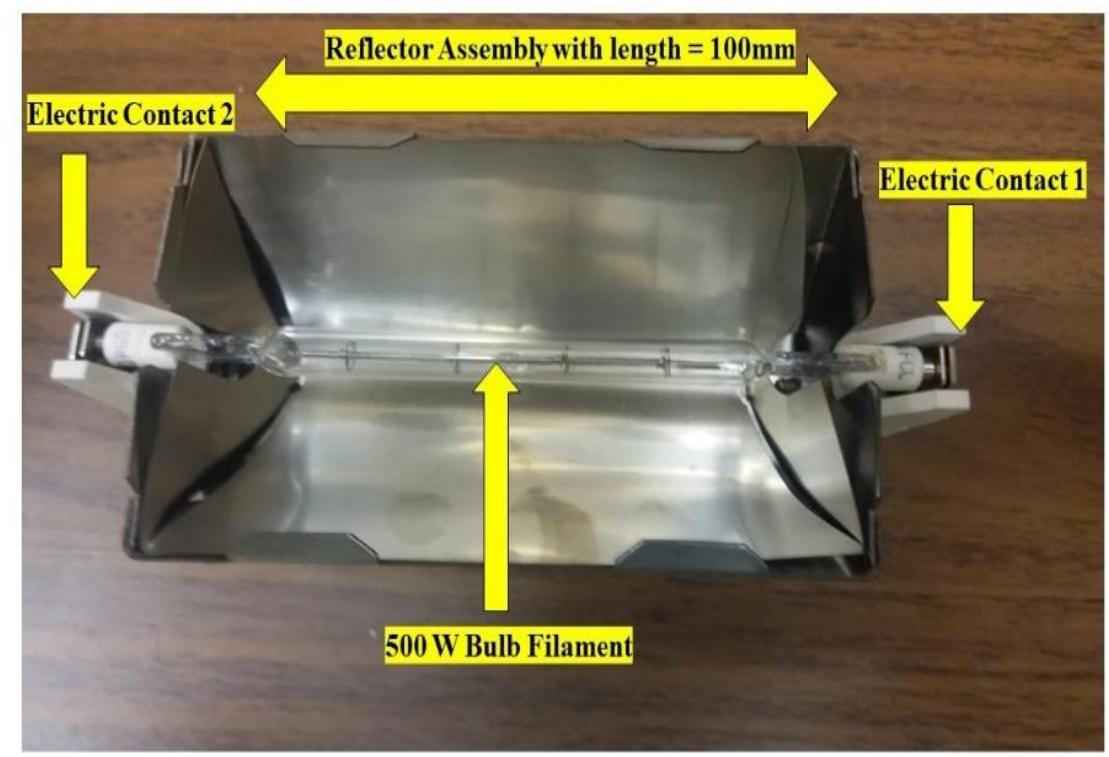

Figure 16: PHOTOGRAPH OF THE QUARTZ LAMP HEATER ASSEMBLY.

The lamp was powered using a variable AC transformer 'variac' that controlled the power delivered to the bulb; hence, controlling the total amount of radiating energy.

\subsubsection{Temperature Measurement}

Temperature measurement was important in SEY experiment to better understand the impact of baking samples on the SEY. A type-K thermocouple was mounted on the sample surface to measure its temperature. A type-K thermocouple feedthrough (consisting of Chromel and Alumel) was used to transmit a thermocouple's voltage-difference signal from inside a vacuum chamber to the data acquisition system. Figure 17 illustrates the thermocouple feedthrough used in this experiment [55]. 


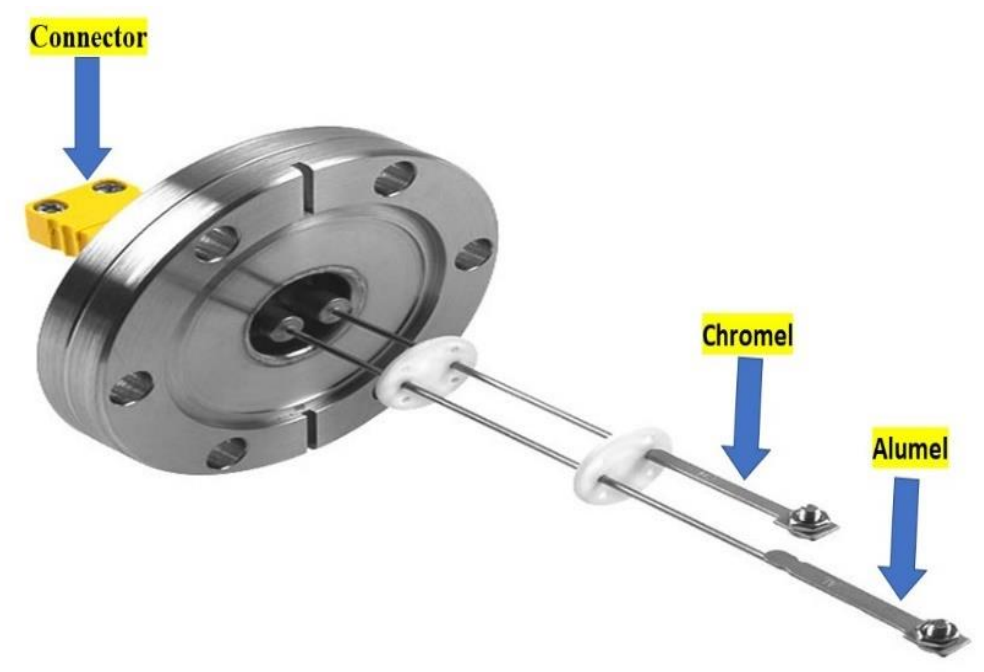

Figure 17: 2.75" CF FLANGE THERMOCOUPLE FEEDTHROUGH.

The vacuum side of the feed-through terminated in thermocouple lugs and hooked up to a thermocouple wire (K-type) that connected the sample to the feedthrough to compensate for the distance between the feedthrough and the sample. The air side connectors of the feed-through were used to connect to the NI temperature DAQ device, which was connected to the PC via a USB port for data logging. 


\section{Chapter 3}

\section{Surface Treatment Methods}

\subsection{Samples Tested and Installation of Samples}

The samples tested in these experiments were copper, silver, stainless steel, aluminum 6061 (Al), monel, nickel/cobalt, and invar. The list of samples was received from the MURI team's commercial aerospace partners. All of the samples tested in this experiment were materials of interest to VEDs, and were procured from Good Fellow USA, a leading vendor of scientific materials. The sample size of copper, silver, nickel/cobalt, monel, and aluminum samples was $50 \mathrm{~mm} \times 50 \mathrm{~mm} \times 2 \mathrm{~mm}$ and the sample size of invar was $25 \mathrm{~mm}$ x $25 \mathrm{~mm} \times 2 \mathrm{~mm}$.

All samples had $2 \mathrm{~mm}$ diameter holes drilled in them in order to mount them on the sample holder. Two holes were drilled in samples that were subjected to baking. The second hole was used for connecting a thermocouple to the sample for measuring the surface temperature.

A highly conductive path for flow of beam and target current is desirable which is accomplished by mounting the sample on copper mounts. Care was taken while installing the sample so that copper mount did not touch the sample holder (brass extension), which is grounded with the chamber wall. Ceramic screws are used to isolate the sample from ground. The sample was connected to the copper mount using a metal screw. 


\subsection{Surface Treatments}

The surface treatments adopted in this study were:

1 As received (no cleaning method applied)

2 Roughing with sandpaper and cleaning with methanol

3 Ultrasonic cleaning-I

4 Revised ultrasonic cleaning-II

5 Thermal treatment/baking.

The following is a brief description of the treatment methods listed above:

\section{1) As received}

The 'as received' samples were not subjected to any kind of surface treatment. These samples were introduced into the chamber in the same state as they were received from the vendor. The importance of the 'as received' sample will be explained in the next chapter.

2) Roughing with sandpaper and cleaning with methanol

In this treatment method, samples were sanded with 600 grit sandpaper and cleaned with methanol before placing them inside the vacuum chamber. Mechanical sanding was performed in the same direction for all samples.

3) Ultrasonic cleaning-I

This ultrasonic cleaning-I was a three-step, industrial-prescribed cleaning method as follows:

- Five-minute ultrasonic treatment with acetone (semiconductor grade) 
- Five-minute ultrasonic agitation by aviation industry approved 815 GD mildly alkaline pure detergent

- Five-minute ultrasonic rinsing with DI (deionized) water.

After completing the above mentioned three steps of ultrasonic treatment, the sample was air-dried before placing it into the vacuum chamber. Figure 18 shows the three ultrasonic cleaners used for ultrasonic treatment.

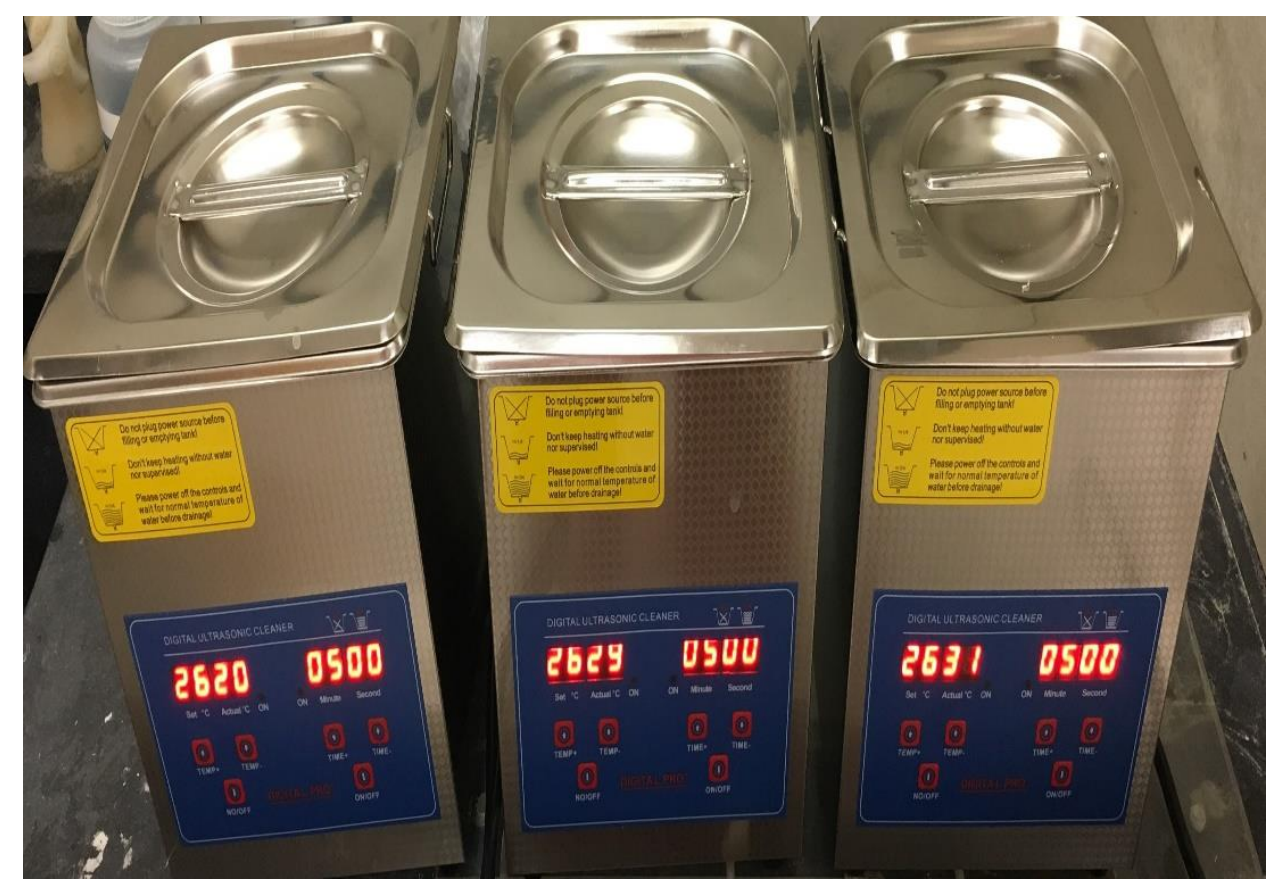

Figure 18: ULTRASONIC CLEANERS, MODEL: JPS-08A.

Ultrasonic cleaners include digital controls that allow for setting the temperature and time duration for ultrasonic cleaning.

Ultrasonic cleaning is a process that uses ultrasound to agitate a fluid. In an ultrasonic cleaner, the object to be cleaned is placed in a chamber containing a suitable solution (in an aqueous or organic solvent, depending on the application). In aqueous cleaners, surfactants (e.g., detergents) are often added to permit dissolution of non-polar 
compounds such as oils and greases. An ultrasound generating transducer built into the chamber or lowered into the fluid produces ultrasonic waves in the fluid by changing size in concert with an electrical signal oscillating at an ultrasonic frequency. This creates compression waves in the liquid of the tank that 'tears' the liquid apart, leaving behind many millions of microscopic 'voids'/'partial vacuum bubbles' (cavitations). These bubbles collapse with enormous energy; temperatures and pressures, however, are so less that they do no more than clean and remove surface dirt and contaminants. The higher the frequency, the smaller the nodes between the cavitation points, which allows for cleaning of features with more intricate detail.

\section{4) Ultrasonic cleaning-II}

The motivation to use ultrasonic cleaning-II was due to the formation of a detergent film that could be observed on the surface of the sample after an ultrasonic cleaning-I. In order to increase water cavitation around the samples to better remove detergent residue, the ultrasonic cleaning protocol-I was revised as follows:

- 5 minutes ultrasonic treatment with acetone (the first step is the same as Ultrasonic-I).

- 5 minutes ultrasonic treatment with Brulin's 815 GD detergent diluted with DI water. The dilution ratio was kept at $90 \%$ DI water and $10 \%$ detergent.

- In place of ultrasonic rinsing, three beakers filled with DI water were utilized. After cleaning with detergent, a sample was placed in beaker 1, agitated for $1 \mathrm{~min}$, moved to breaker 2, agitated for $1 \mathrm{~min}$ and then moved to final beaker 3 , agitated for $1 \mathrm{~min}$. 
- The sample was then thoroughly blow-dried with nitrogen $\left(\mathrm{N}_{2}\right)$ before installing it in the chamber for experiment.

\section{5) Thermal treatment/baking}

A thermal treatment of the samples was performed under high vacuum conditions. There was a single instance of baking the sample while the vacuum chamber was vented to atmospheric pressure which will be explicitly mentioned in the next chapter. Samples were baked in the chamber using a quartz lamp heater to remove adsorbed gas atoms and oxidation layers. The lamp was attached to a variac to control the power delivered to the lamp. The electrical power delivered to the filament was $\mathrm{P}_{\text {in }}=\mathrm{IV}$ where $\mathrm{I}$ is the steadystate current and $\mathrm{V}$ is the voltage across the filament.

The power leaving the filament due to conduction and radiation is

$$
\mathrm{P}_{\text {out }}=\mathrm{C}_{\mathrm{c}}\left(\mathrm{T}-\mathrm{T}_{\mathrm{o}}\right)+\mathcal{E}_{\mathrm{eff}} \sigma \mathrm{A}\left(\mathrm{T}^{4}-\mathrm{To}^{4}\right)
$$

where,

$\mathrm{C}_{\mathrm{c}}$ is coefficient of conduction,

$\mathrm{T}$ is filament temperature,

$\mathrm{T}_{\mathrm{o}}$ is the temperature of the lamphouse,

$\sigma=5.64 * 10^{-8} \mathrm{~J} / \mathrm{m}^{2} \mathrm{~K}^{4} \mathrm{~s}$ is the Stephan- Boltzmann constant,

A is the surface area of the filament $=280 * 10^{-6} \mathrm{~m}^{2}$, and

$\varepsilon_{\text {eff }}=0.44$ is the weighted average of wavelength-dependent emissivity [56].

Since in steady-state the input power must be equal to the output power, the electrical parameters of the filament are related to the temperature. 
Figure 19 shows a photograph of the IR heater turned on while the vacuum chamber is exposed to atmospheric pressure, i.e. 660 Torr in Albuquerque, NM. The thermocouple wire for temperature measurement is covered with ceramic beads to protect it from the high temperature.

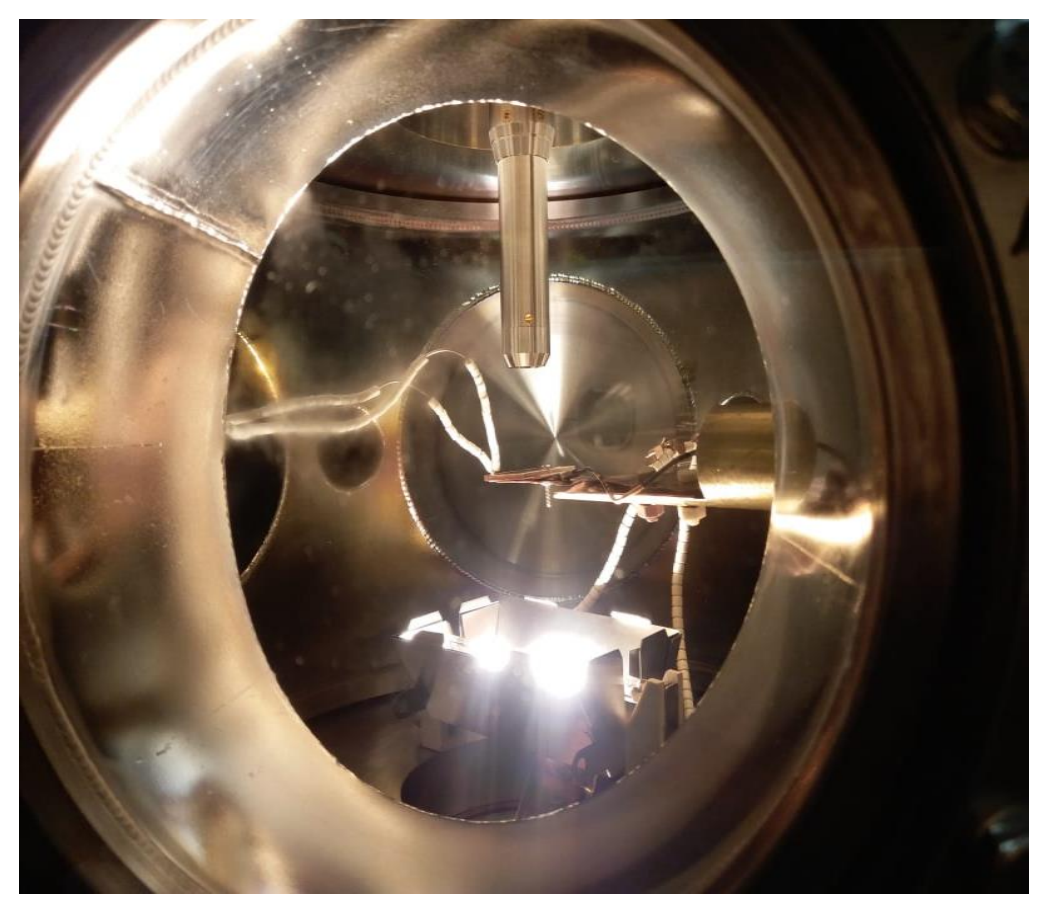

Figure 19: PHOTOGRAPH OF THE VACUUM CHAMBER WITH QUARTZ LAMP TURNED ON.

The contaminants that must be considered for this experiment were adsorbed gases, water molecules, and oxidation layers. These can be removed from the surface through heating. It has been mentioned in [57] that heating a metal to $425^{\circ} \mathrm{C}$ in a good vacuum will remove all but the last few monolayers, whereas, heating to $1000^{\circ} \mathrm{C}$ will remove all but the last one.

The formation of contaminants on the surface of the sample prior to placing them in vacuum was uncontrollable because the monolayer formation time at atmospheric pressure 
is nanoseconds. Therefore, it is always good to use heating for cleaning the surfaces from contaminants. 


\section{Chapter 4}

\section{Experimental Results}

This chapter presents SEY experimental results on different materials of interest to VEDs and shows SEY variation with different surface treatments. SEY $(\delta)$, as mentioned previously, is determined by measuring the ratio of the beam and target currents: $\delta=\mathrm{I}_{\mathrm{t}} / \mathrm{I}_{\mathrm{b}}$, where $\mathrm{I}_{\mathrm{t}}$ represents target current and $\mathrm{I}_{\mathrm{b}}$ represents primary beam current. SEY measurements were made for normal incidence of the electron beam unless stated otherwise. There were a few measurements made at oblique angles of incidence that will be shown explicitly. For measurements with varying angle of incidence of primary electrons, angle was referenced relative to the surface normal. The angle of incidence of the electron beam was changed by rotating the sample using a rotary seal feedthrough on the sample mount.

In all experiments, SEY vs. beam energy was measured with the beam energy varied from $10 \mathrm{eV}$ to $1000 \mathrm{eV}$, i.e. the low energy regime. Some experiments were performed using varying beam energy from $10 \mathrm{eV}$ to $1980 \mathrm{eV}$, and the results for those experiments are shown explicitly later in this chapter. Copper, being the baseline material, has been tested more frequently than other materials in this thesis work. Since there is already a significant amount of published work related to SEY measurements on copper, it was chosen in order to make comparisons with previously reported SEY measurements. 


\subsection{Experimental Parameters}

All samples tested in this research were mounted on the sample holder described in chapter 2. The operating distance - the distance from the electron gun aperture to the sample - used in these experiments was $40 \mathrm{~mm}$.

The sample was negatively biased to measure the target current. Since both the electron gun voltage and the sample bias are referenced to the grounded vacuum chamber wall, the energy of an electron incident on a sample surface is decreased by an amount equal to the applied negative bias. This negative bias was compensated for in SEY calculations by subtracting the bias from the beam energy. For example, if the sample is biased at $-20 \mathrm{~V}$ and beam energy hitting the sample surface is $50 \mathrm{eV}$, the effective beam energy hitting on the sample surface is $50 \mathrm{eV}-20 \mathrm{eV}=30 \mathrm{eV}$. If this negative bias is not compensated for in SEY measurements, then a dip in the SEY curve is observed at a point where beam energy equals the negative bias.

In Fig. 20 the impact of applying bias vs. no bias on the copper sample, after the baking is turned off, is shown.

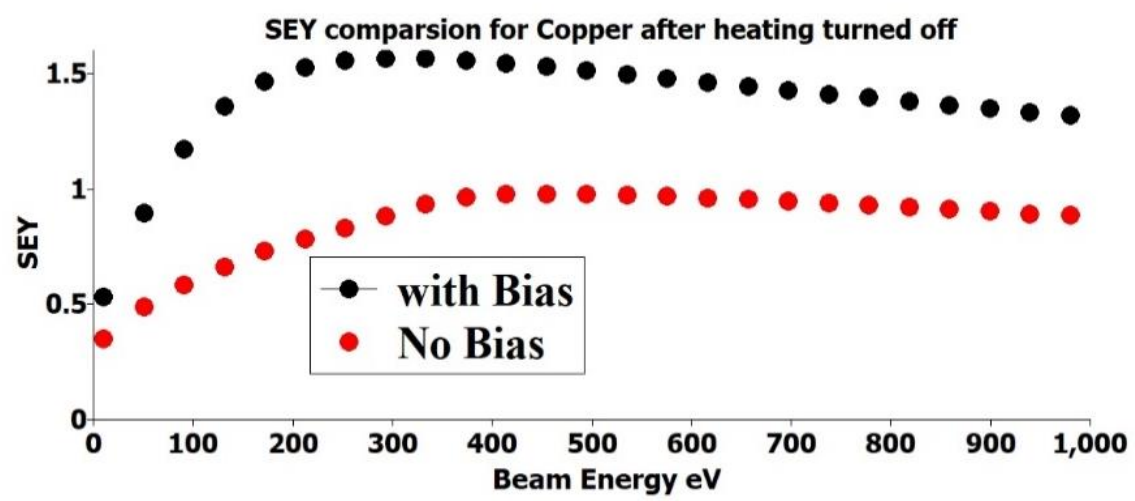

Figure 20: EFFECT OF BIAS VS. NO-BIAS ON SEY OF COPPER. 
The bias applied to the sample was $-20 \mathrm{~V}$, which is the regular bias applied in all the experiments in this research. With no bias applied to the sample, SEY was less than 1 throughout the entire beam energy scan.

SEY experiments were performed by varying beam energy from the lowest to highest value while measuring SEY at each specific energy. For each beam energy, first the beam currents and then the target currents were recorded. For each data point, an average of 3 samples was taken and 50 steps were required in going from $10 \mathrm{eV}$ to $1000 \mathrm{eV}$

The electron dose (D) is defined as the charge received by the sample per unit area,i.e., D $=\mathrm{Q} / \mathrm{A}$ where $\mathrm{Q}=\mathrm{I}_{\mathrm{b}} \times \mathrm{t}$ is the total charge incident on the sample surface, $\mathrm{A}$ is the area of sample surface exposed to electron beam, and $t$ is the exposure time that the beam impinges of the sample. During SEY measurement, a dose of $10^{-6} \mathrm{C} / \mathrm{mm}^{2}$ was maintained to have a negligible effect in terms of electron beam conditioning [37][58]. Table 5 summarizes the important experimental parameters.

Table 5: Experimental parameters.

\begin{tabular}{|c|c|}
\hline Parameter Name & Parameter Value \\
\hline Dose & $10^{-6} \mathrm{C} / \mathrm{mm}^{2}$ \\
\hline Positive Bias & $100 \mathrm{~V}$ \\
\hline Negative Bias & $20 \mathrm{~V}$ \\
\hline Operating Distance & $40 \mathrm{~mm}$ \\
\hline Spot size & $1 \mathrm{~mm}$ \\
\hline Vacuum Pressure & $10^{-8} \mathrm{Torr}$ \\
\hline Electron beam energy & $10 \mathrm{eV}-1000 \mathrm{eV}$ \\
\hline
\end{tabular}




\subsection{Materials and Cleaning Protocol Checklist}

Table 6 summarizes the materials and cleaning protocols used in this experimental study.

Table 6 : Checklist for materials and cleaning protocols employed in the SEY experiment.

\begin{tabular}{|c|c|c|c|c|c|c|c|}
\hline \multirow{2}{*}{$\begin{array}{c}\text { Materials } \\
\text { tested }\end{array}$} & \multicolumn{7}{|c|}{ Cleaning Protocols } \\
\hline & As -received & Ultra - I & Ultra-II & $\begin{array}{c}\text { Roughing } \\
+\end{array}$ & $\begin{array}{l}\text { Ultra- } \\
\text { I,II + } \\
\text { baking }\end{array}$ & $\begin{array}{l}\text { As - } \\
\text { received } \\
\text { baked }\end{array}$ & $\begin{array}{l}\text { Baking } \\
\text { in air + } \\
\text { vacuum }\end{array}$ \\
\hline Copper & $\checkmark$ & $\checkmark$ & $\checkmark$ & $\checkmark$ & $\checkmark$ & $\checkmark$ & $\checkmark$ \\
\hline Siver & $\checkmark$ & $\checkmark$ & $\checkmark$ & $\checkmark$ & $\checkmark$ & $x$ & $x$ \\
\hline Aluminum & $\checkmark$ & $\checkmark$ & $x$ & $x$ & $x$ & $x$ & $x$ \\
\hline $\begin{array}{c}\text { Nickel/Cobalt } \\
\text { Alloy }\end{array}$ & $x$ & $\checkmark$ & $x$ & $x$ & $x$ & $x$ & $x$ \\
\hline Monel & $x$ & $\checkmark$ & $x$ & $x$ & $x$ & $x$ & $x$ \\
\hline Invar & $x$ & $\checkmark$ & $x$ & $\checkmark$ & $x$ & $x$ & $x$ \\
\hline
\end{tabular}

It is evident from Table 6 that copper, being a baseline and important material for VEDs, was subjected to more measurements compared to other materials. In addition, our MURI team's DFT groups are focused on copper for SEY simulation. After copper, silver was subjected to more SEY measurements than any other material. 


\subsection{Experimental Results}

In this section SEY measurements of copper are presented, and a comparison of the SEY of different materials is made. Cleaning protocols are shown subsequently.

\subsubsection{SEY of Copper}

The following are SEY measurements of as-received copper, and SEY comparison of copper cleaned with different treatment methods. Differences in SEY for different cleaning protocols highlight that SEY is a surface-specific phenomenon.

1) As received sample

The SEY of an as-received sample is important to the space industry because not all components installed in a satellite payload are surface cleaned. Components in the RF chain of satellite payload, such as filters that are prone to MPB do not receive any kind of surface treatment. Therefore, it is of interest to measure SEY of as-received materials and to compare it with SEY of clean surfaces. Figure 21 shows the SEY of 'as received' copper samples. The as-received samples were installed in the vacuum chamber with minimum exposure to air. The samples were handled with care by using latex gloves so that handprints are avoided while installing the sample. As can be seen in Fig. 21, the first crossover point $\left(\mathrm{E}_{1}\right.$, where $\left.\delta=1\right)$ for all the as - received samples is at low primary beam energy, i.e. $\sim 30 \mathrm{eV}$. The first crossover point $\mathrm{E}_{1}$ and the frequency - gap product (fd) are important for multipactor analysis and prediction that involves two surfaces. With lower frequency-gap products (fd), there is a physical cutoff for which the multipactor criteria for increase in electron population cannot be met. This is referred to as $\mathrm{fd}_{\min }$ and it is principally dependent on the $\mathrm{SEY}$, i.e., $\mathrm{fd}_{\min }(\mathrm{GHz}-\mathrm{mm})$ is proportional to $\sqrt{E 1}$ [3]. The $\mathrm{SEY}$ curve 
with point $E_{1}$ moved to higher energies is desired to improve the multipactor margin/threshold in space-based RF devices. The $\mathrm{E}_{1}$ point of as-received samples is at low primary energy, which is undesirable .

In Fig. 21 the SEY measured on four copper samples is shown - two from batch 1 and two from batch 2 where different "batch" numbers indicate samples received from the manufacturer on different days. Copper of purity level $99.99+\%$ and part number CU000766 was procured from Good Fellow USA for all SEY measurements (copper related) shown in this thesis.

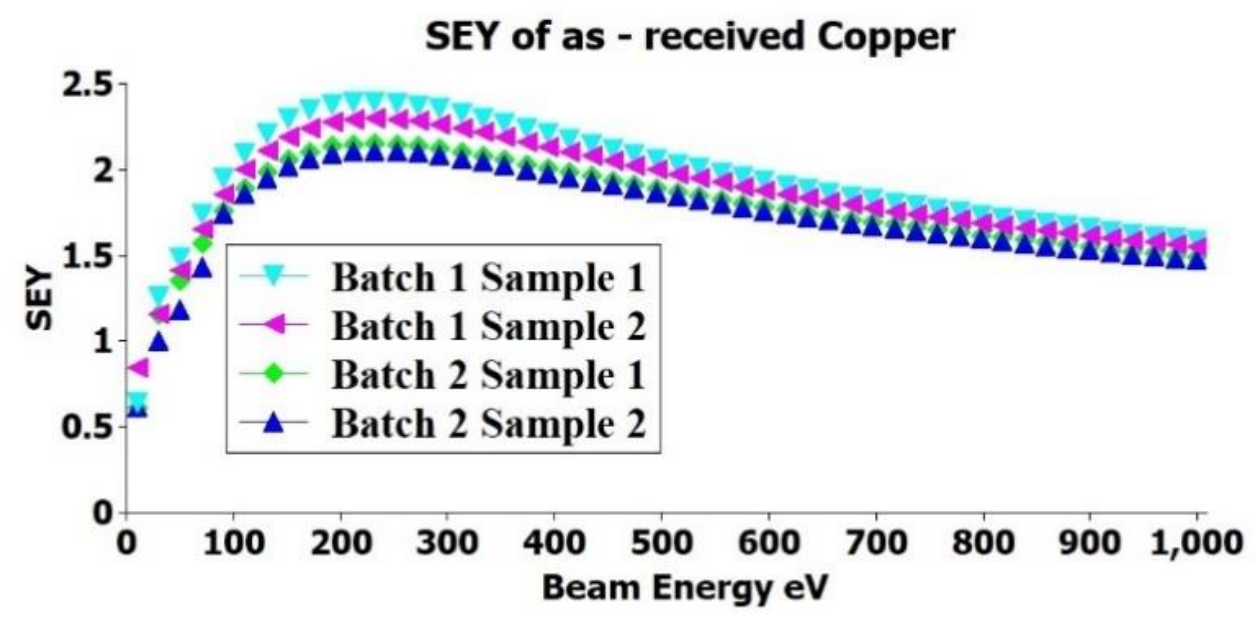

Figure 21: SEY OF AS-RECEIVED COPPER.

For this experiment involving as-received copper samples, the reason for selecting different batches was to study the range of variation in SEY. The result showed that SEY was consistently greater than 2 in all four samples, which is in good agreement with already published data of the SEY of as-received copper samples [25][37][43]. The maximum SEY $\left(\delta_{\max }\right)$ varied in the range from 2.39 (batch 1, sample 1) to 2.14 (batch 2, sample 2) at a corresponding $\mathrm{E}_{\max } \approx 230 \mathrm{eV}$. 
The variation in SEY of the same material, i.e., copper, was apparently due to sample handling procedures, intrinsic differences among the as-received samples within the same batch, and differences between batches. The as-received SEY for a silver sample is shown in section 4.3.2 in order to compare as-received SEY with various cleaning methods.

2) Ultrasonic- I cleaned sample

Figure 22 shows the SEY measured on the ultrasonic-I treated copper sample by scanning the beam energy over the spectrum of $10 \mathrm{eV}$ to $1000 \mathrm{eV}$. Comparing Figs. 21 and 22, it can be seen that the Ultrasonic-I protocol decreases the measured SEY of copper.

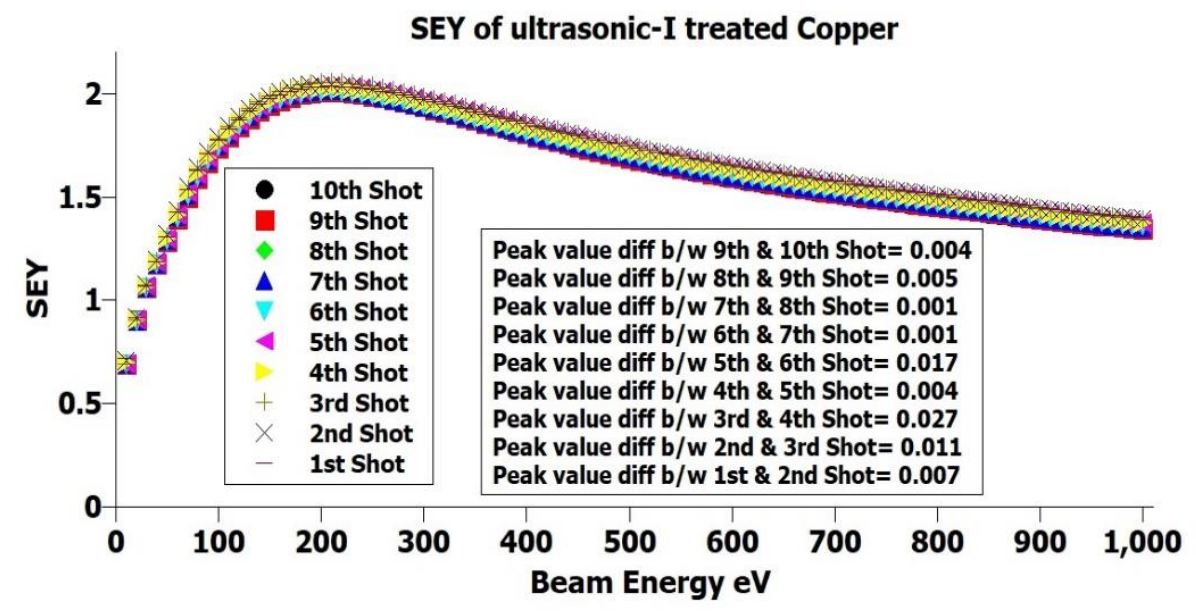

Figure 22: SEY OF ULTRAOSNIC-I COPPER.

In the experiments presented in Fig. 22, 10 SEY measurement shots were made by maintaining the dose around $10^{-6} \mathrm{C} / \mathrm{mm}^{2}$ for each shot. This was done to make sure that there was no coupling of the electron scrubbing in SEY measurements [25] [37][58]. The SEY decreases for doses greater than $10^{-6} \mathrm{C} / \mathrm{mm}^{2}$ and its maximum stabilizes for doses greater than $1 * 10^{-3} \mathrm{C} / \mathrm{mm}^{2}$. This effect is permanent when the surface is kept under vacuum. It can be seen from Fig. 22 that the SEY curves are almost laying over each other 
and are equivalent across the entire energy spectrum. The $\delta_{\max }$ of Ultrasonic- 1 copper $\approx 2$ at corresponding $\mathrm{E}_{\max }=200 \mathrm{eV}$ for almost all the 10 curves/shots. The SEY variation for these measurements is under $5 \%$.

Figure 23 depicts the SEY results for combination of Ultrasonic-I and beam conditioning at $500 \mathrm{eV}$ treated sample. Beam conditioning has also been reported in the literature as one method for decreasing SEY.

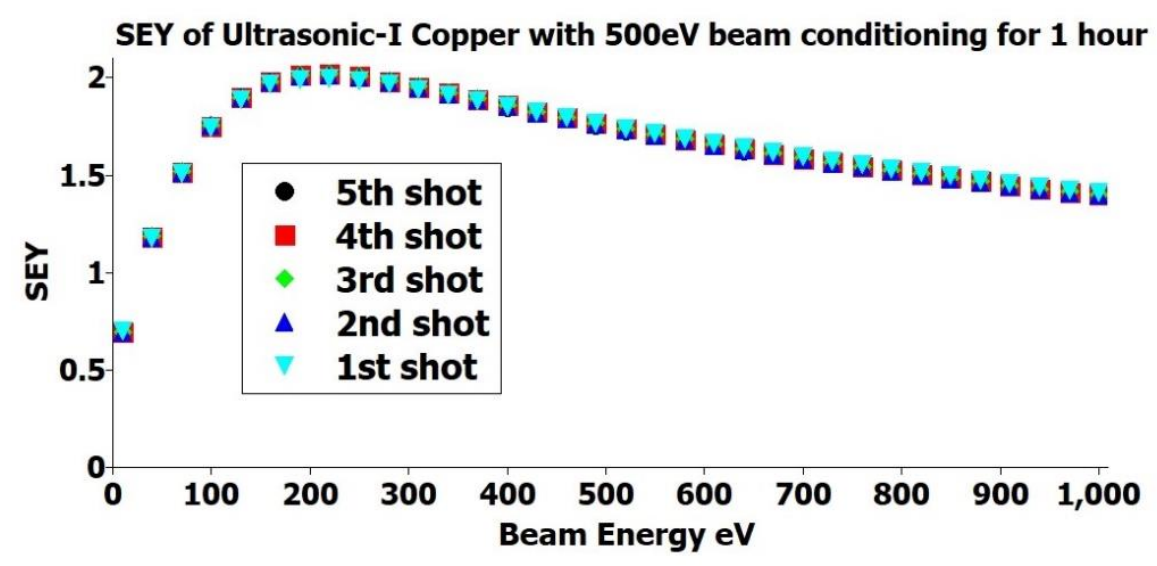

Figure 23: SEY OF COPPER WITH ULTRASONIC-I + 500 EV OF BEAM CONDITIONING.

Electron beam conditioning at $500 \mathrm{eV}$ for one hour was performed with low beam current of the order of $0.5 \mu \mathrm{A}$ to $1 \mu \mathrm{A}$ on the Ultrasonic-I treated sample. The dose for this experiment was again controlled to be around $10^{-6} \mathrm{C} / \mathrm{mm}^{2}$. Five SEY measurements were made, and it can be seen that the SEY curves lie exactly over each other, which again supports the important point that dose-effect does not appear to be affecting these SEY measurements. It should also be noted that beam conditioning the Ultrasonic-I copper sample did not make any significant difference to the SEY when compared with Ultrasonic- 
I treated sample alone. The $\delta_{\max }$ and $E_{\max }$ are almost the same for Ultrasonic-I and Ultrasonic-I + beam conditioning. The $\delta_{\max }=1.99$ at corresponding $\mathrm{E}_{\max }=200 \mathrm{eV}$.

Beam conditioning with $750 \mathrm{eV}$ beam energy was performed on the Ultrasonic-I copper and the SEY results are shown in Fig. 24. It can be seen in Figs. 23 and 24 that there is no significant decrease in SEY with beam conditioning, which may be because low beam currents were employed in these experiments. For $750 \mathrm{eV}$ beam conditioning, as shown in Fig. 24, the $\delta_{\max }=2.07$ at corresponding $\mathrm{E}_{\max }=200 \mathrm{eV}$. Conditioning with a $750 \mathrm{eV}$ beam generated higher SEY of copper than conditioning with a $500 \mathrm{eV}$ beam.

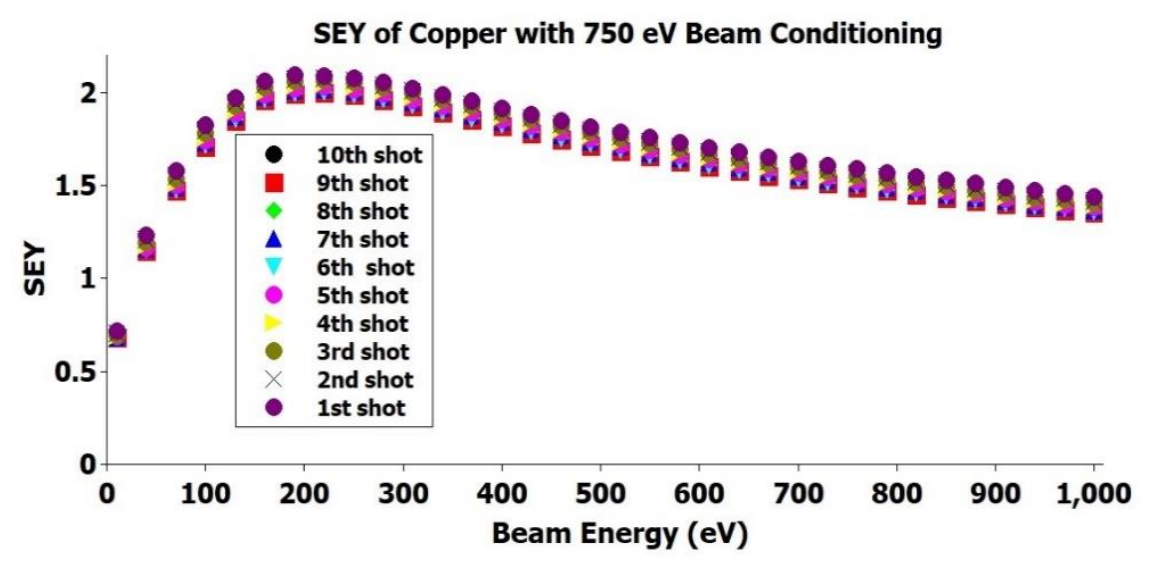

Figure 24: SEY OF COPPER WITH 750 EV BEAM CONDITIONING.

It is evident from Fig. 24 that all 10 scans resulted in SEY curves lying over each other, which is consistent with the Ultrasonic-I and Ultrasonic-I $+500 \mathrm{eV}$ beam conditioning data. It can be concluded that beam conditioning with low currents did not change the SEY of the Ultrasonic-I cleaned sample.

The dose was slightly increased from $10^{-6} \mathrm{C} / \mathrm{mm}^{2}$ to show the effect of increasing dose on the SEY of the ultrasonic-I-cleaned copper. Figure 25 shows the variation in SEY for an ultrasonic-I cleaned copper as a function of electron dose. 


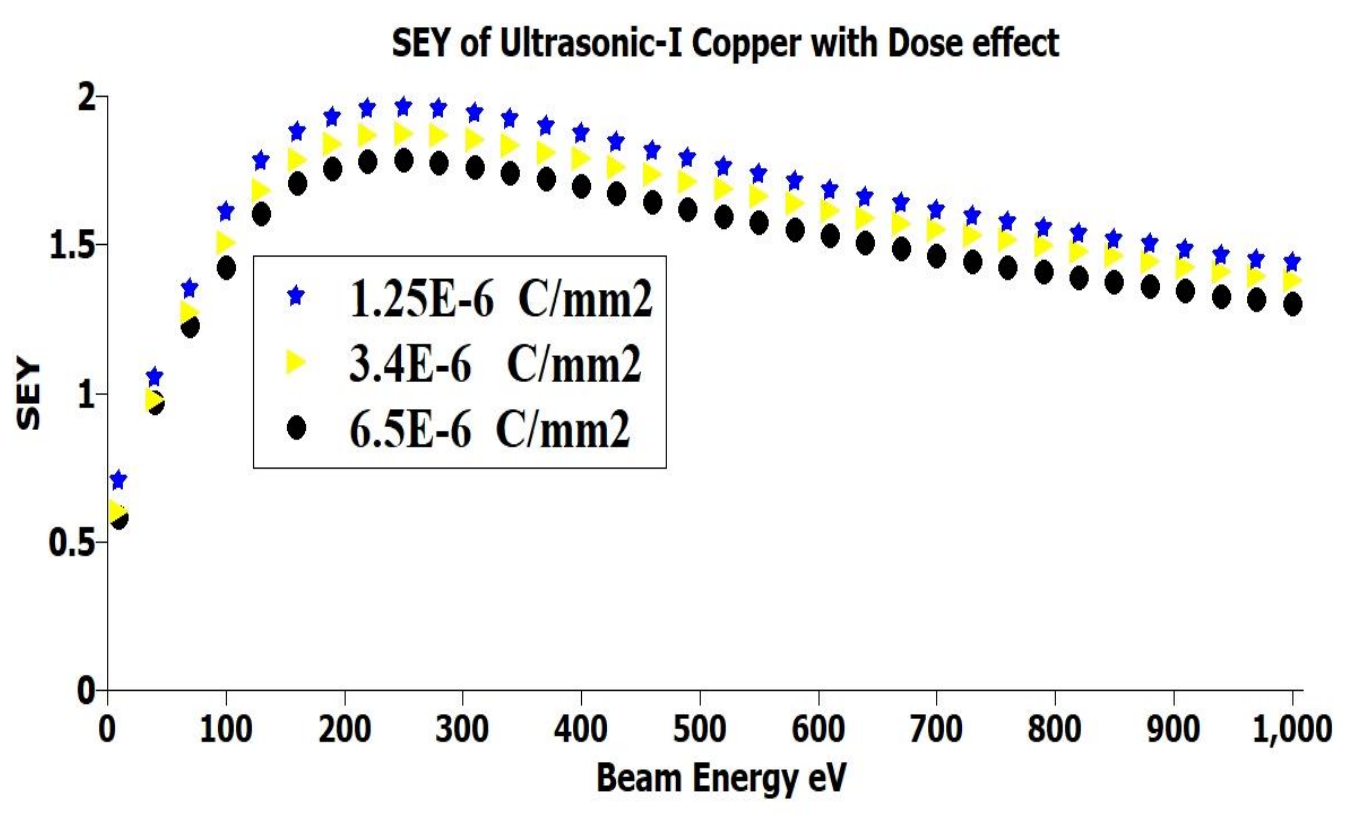

Figure 25: DOSE EFFECT ON ULTRASONIC-I CLEANED COPPER.

As the dose hitting the sample surface increases to more than $10^{-6} \mathrm{C} / \mathrm{mm}^{2}$ the SEY begins to decrease. This is in good agreement with already published data that SEY decreases for doses larger than $10^{-6} \mathrm{C} / \mathrm{mm}^{2}$ [37].

Figure 26 illustrates the SEY of ultrasonic-I-cleaned copper vs. angle of electron beam incidence. The angle of incidence is varied from 0 to 30 degrees by rotating the sample inside the vacuum chamber through rotary seal. SEY increases with primary electron energy under an oblique angle of incidence. The reason for this increase can be explained as follows: if primary electrons falling normally on the surface release secondary electrons at a mean depth $\mathrm{x}_{\mathrm{m}}$, when the angle of incidence changes to angle $\theta$ this depth becomes $\mathrm{x}_{\mathrm{m}}$ $\cos \theta$ [22]. Fewer secondary electrons are now absorbed before they reach the surface which is why SEY increases with oblique angles. 


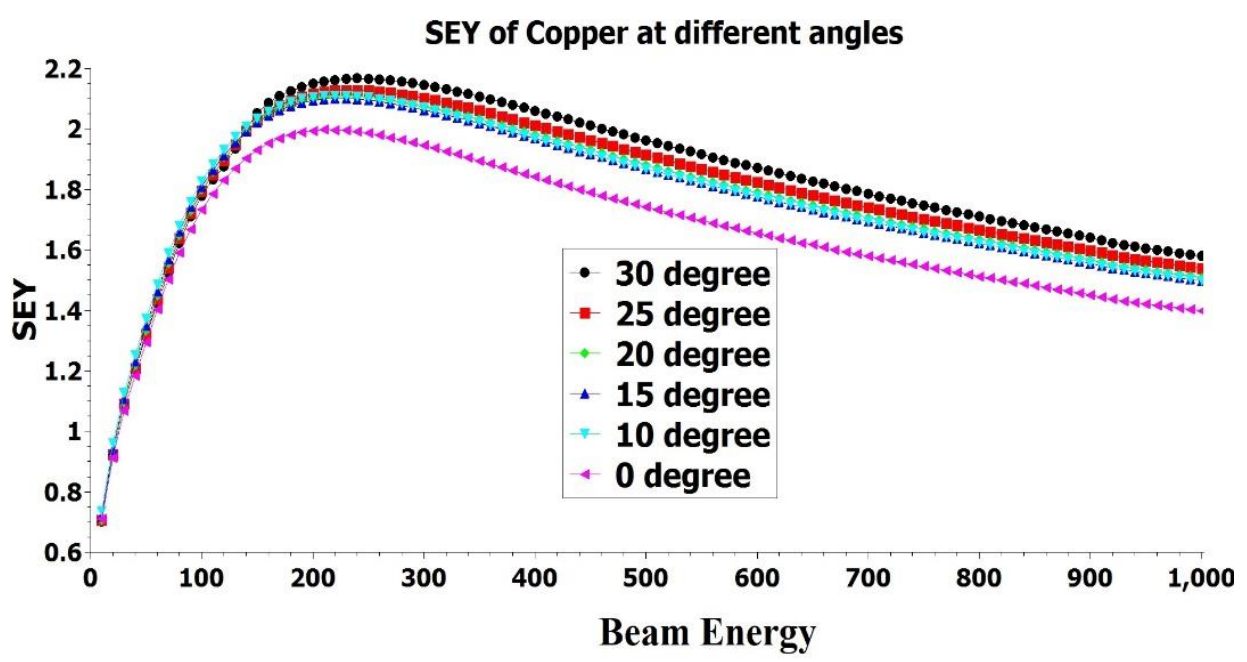

Figure 26: VARIATION IN SEY WITH DIFFERENT ANGLES OF INCIDENCE.

It is clear from the figure that as the angle of incidence is increased, SEY of Ultrasonic-I copper also increases. This is also in good agreement with the published data [21]. It should be noted here that $0^{\circ}$ represents normal incidence and $90^{\circ}$ represents grazing incidence.

3) Comparison of ultrasonic-I and ultrasonic-II protocols

Figure 27 shows a comparison of SEY measurements for Ultrasonic-I and Ultrasonic-II protocols applied to copper samples. The Ultrasonic-I protocol was revised to UltrasonicII in order to gain more-efficient cleaning of the surface. The detailed difference between the Ultrasonic-I and Ultrasonic-II cleaning protocol has already been discussed in detail in chapter 3 . The peak SEY, $\delta_{\max }$, of three copper samples cleaned with Ultrasonic-II varied less than $3 \%$ from shot to shot. In order to test for repeatability, SEY measurements were performed on three samples from the same batch cleaned with the Ultrasonic-II protocol. It can be seen in Fig. 27 that the peak value of SEY is reduced with the Ultrasonic-II protocol as compared to the Ultrasonic-I protocol. It will be shown later in this chapter that 
baking in vacuum to $300^{\circ} \mathrm{C}$ for 3 hours reduces the $\delta_{\max }$ difference between Ultra-I and Ultra-II cleaned copper samples.

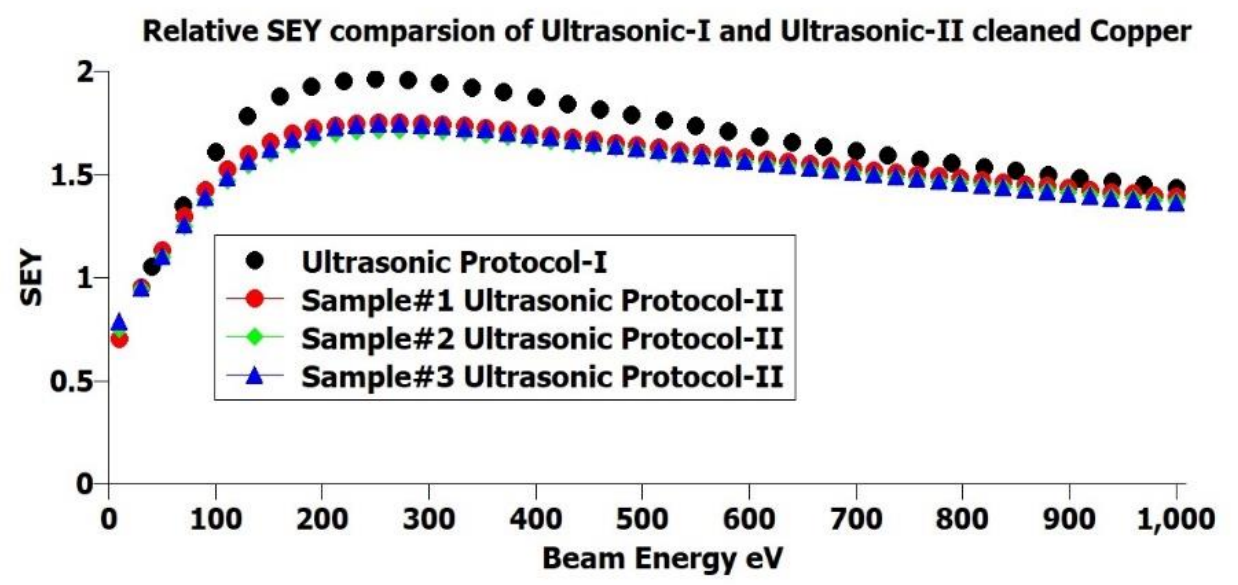

Figure 27: COMPARISON OF ULTRASONIC-I AND ULTRASONIC-II PROTOCOLS.

These are the observations related to Fig. 27:

1) The $\delta_{\max }$ of the ultrasonic-II protocol corresponding to sample \#1 and sample \#3 is 1.75 and 1.74 , respectively, while $\delta_{\max }$ of sample \#2 is 1.70 , which is a minimum of the three samples tested for Ultrasonic-II at corresponding $\mathrm{E}_{\max } \approx 300 \mathrm{eV}$.

2) Two important SEY curve parameters, i.e., $E_{\max }$ and $E_{1}$, are translated to higher electron beam energies with Ultrasonic-II cleaning protocol as compared to asreceived copper samples.

3) The lowering of $\delta_{\max }$ for the Ultrasonic-II protocol shows that it appears to have done a better job of cleaning and rinsing as compared to the ultrasonic-I protocol for copper. The SEY of sample \#1, sample \#2, and sample \#3 cleaned using Ultrasonic-II is presumably different because of uncontrolled variables that can change the surface behavior to an incident electron beam. 
So far we have seen that the Ultrasonic-II protocol has shown good performance as compared to beam conditioning at low beam currents and the Ultrasonic-I protrocol. It will be shown later in this chapter that the Ultra-II protocol also outperformed roughed + methanol cleaning protocol.

4) Baking Ultrasonic-I and Ultrasonic-II cleaned samples

A typical baking temperature vs. time curve is shown in Fig. 28. The vertical-axis is temperature in degree Celsius while the horizontal-axis is time in minutes.

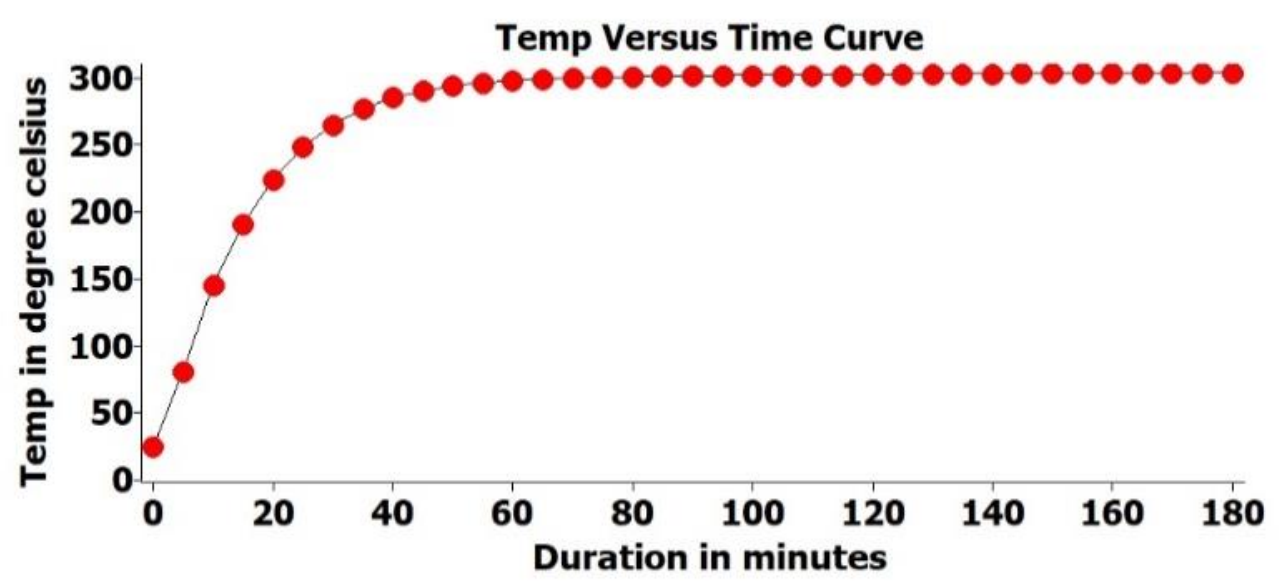

Figure 28: TEMPERATURE VS. TIME CURVE MEASURED BY A CONTACT THERMOCOUPLE ON THE SAMPLE. MEASUREMENT ACCURACY IS APPROXIMATELY $2{ }^{\circ} \mathrm{C}$.

From Fig. 28 it is apparent that a temperature of $\sim 300^{\circ} \mathrm{C}$ was achieved in an hour by operating the variac at around $70 \mathrm{~V} \mathrm{AC}$. The variac is capable of delievering maximum power of $120 \mathrm{~V} \mathrm{AC}$ at the input terminals of the heating lamp. The time required to reach $300^{\circ} \mathrm{C}$ can vary depending on the power applied to the lamp through the variac.

Figure 29 shows the effect of baking to $300^{\circ} \mathrm{C}$ on an Ultrasonic-I-cleaned sample. 


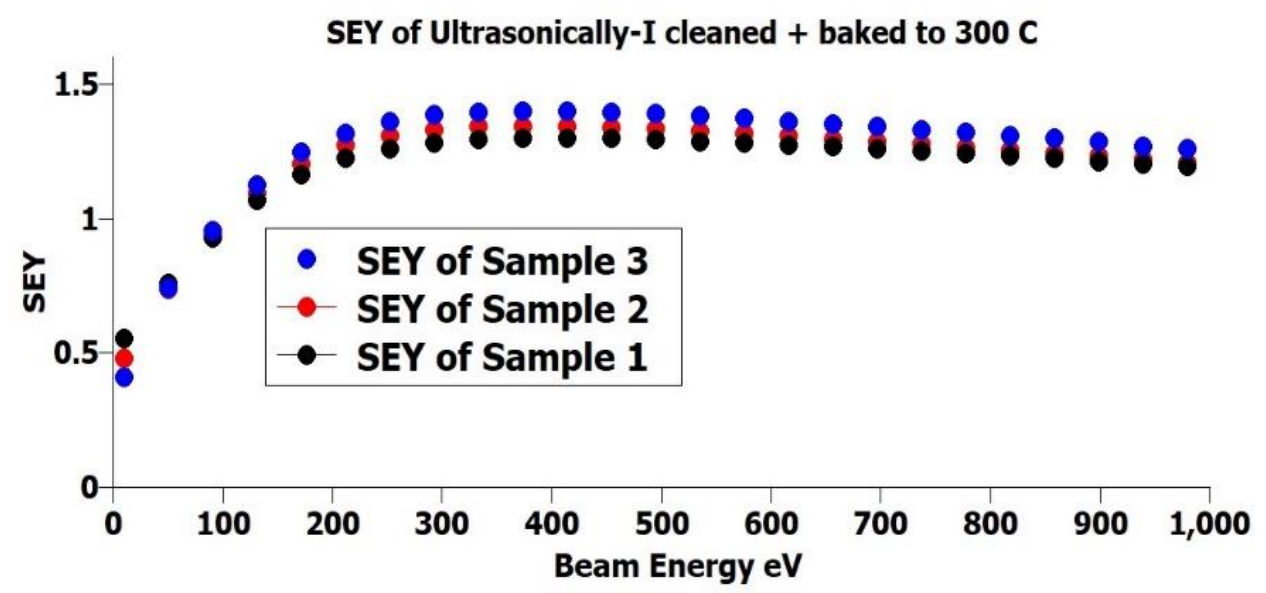

Figure 29: SEY OF COPPER WITH ULTRASONIC-I CLEANED AND BAKED TO 300 C.

Baking to $300^{\circ} \mathrm{C}$ apparently removed much of the adsorbates present on the surface of the sample. Presumably, water was a common adsorbate present on the sample surface and was removed after heating to $300^{\circ} \mathrm{C}$. The presence of water molecules enhances the emissivity (SEY) of a surface, which decreases after baking [37]. Three copper samples were subjected to baking at $300^{\circ} \mathrm{C}$ after receiving Ultrasonic-I treatment. Baking resulted in a significant decrease in the SEY of the Ultrasonic-I treated copper, as can be seen by comparing Figs. 27 and 29. The $\delta_{\max }$ of samples \#1, \#2, and \#3 in Fig. 29 is 1.4, 1.34, and 1.30 , respectively, at corresponding $\mathrm{E}_{\max } \approx 400 \mathrm{eV}$. It should be noted that sample \#1 was tested first and sample \#3 was tested last. The respective increase in SEY for samples \#2 and \#3 can be attributed to poor quality of rinsing in the Ultrasonic-I protocol. It was difficult to remove the soap film that forms on the sample surface with Ultrasonic-I cleaning since a $100 \%$ detergent solution was used without any dilution. The inside body of the ultrasonic cleaner used in process of rinsing with DI water also showed a "cat lick"- 
like detergent film formed on it which did not remove even after rinsing whole body with DI water.

Figure 30 shows the SEY measurement results on Ultrasonic-I $+300^{\circ} \mathrm{C}$ baked copper samples. The beam energy for this experiment is scanned from $10 \mathrm{eV}$ to $1980 \mathrm{eV}$. So far in this thesis work, $\mathrm{E}_{\max }, \delta_{\max }$ and $\mathrm{E}_{1}$ parameters of the $\mathrm{SEY}$ curve were discussed. Figure 30 shows SEY curves (blue and red) with the second crossover point ( $\left.\mathrm{E}_{\mathrm{II}}\right)$.

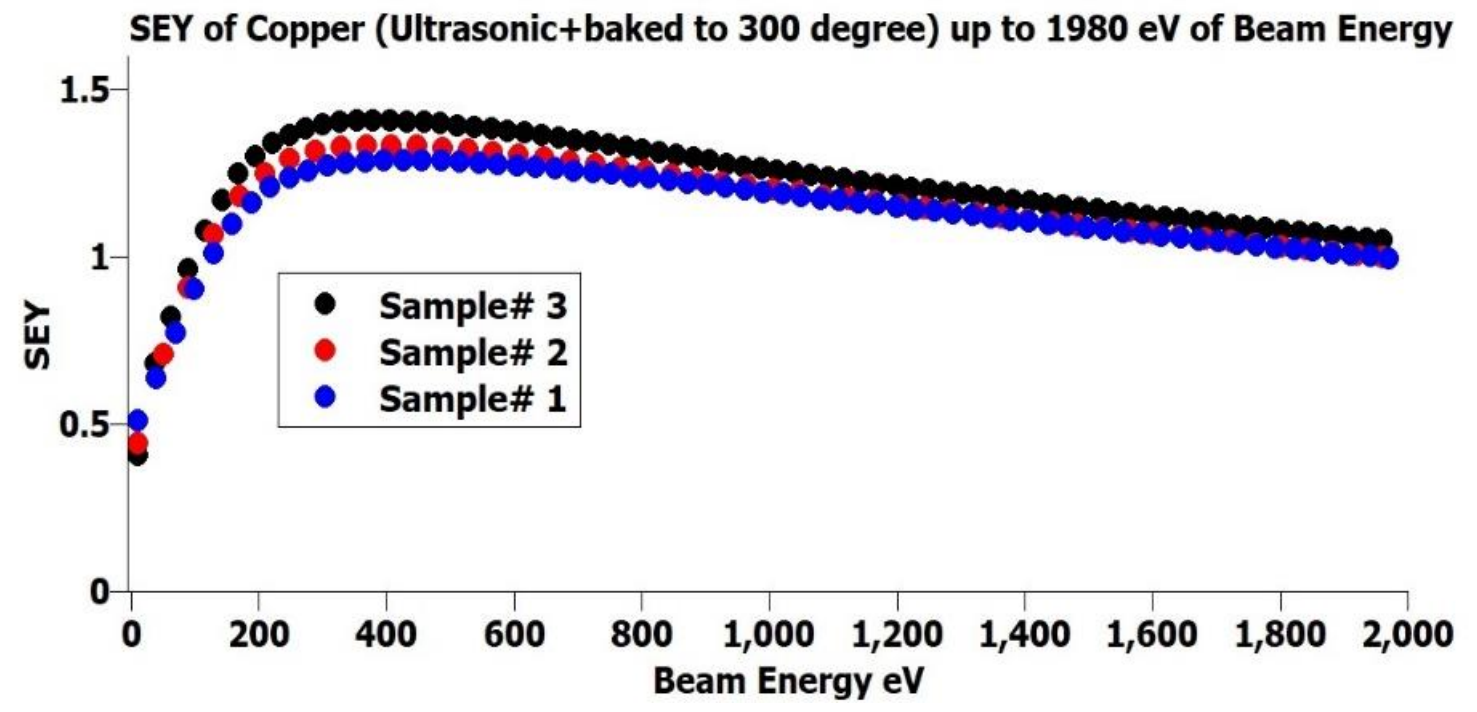

Figure 30: SEY OF ULTRASONIC-I CLEANED COPPER + BAKED TO $300^{\circ} \mathrm{C}$ WITH BEAM ENERGY SCANNED UP TO $1980 \mathrm{eV}$.

Since baking helped reduce SEY, it was of interest to look for the second crossover point, $\mathrm{E}_{\mathrm{II}}$, on the SEY curve. The energy was scanned up to $1980 \mathrm{eV}$ instead of $2000 \mathrm{eV}$ because the sample was biased with a negative 20 volts when measuring target current. Therefore, $20 \mathrm{~V}$ was compensated for while calculating SEY values. Samples \#1 and \#2 showed the second crossover point at corresponding $\mathrm{E}_{\mathrm{II}} \approx 1960 \mathrm{eV}$, while sample \# 3 did not reach the second crossover point and went as close as 1.04 at beam energy $=1980 \mathrm{eV}$. It was 
expected that sample \#3 would show higher SEY than samples \#1 and \#02 due to the rinsing problems discussed before.

Next, two copper samples were cleaned with the Ultrasonic-II protocol and baked to $300^{\circ} \mathrm{C}$. The results are shown in Fig. 31.

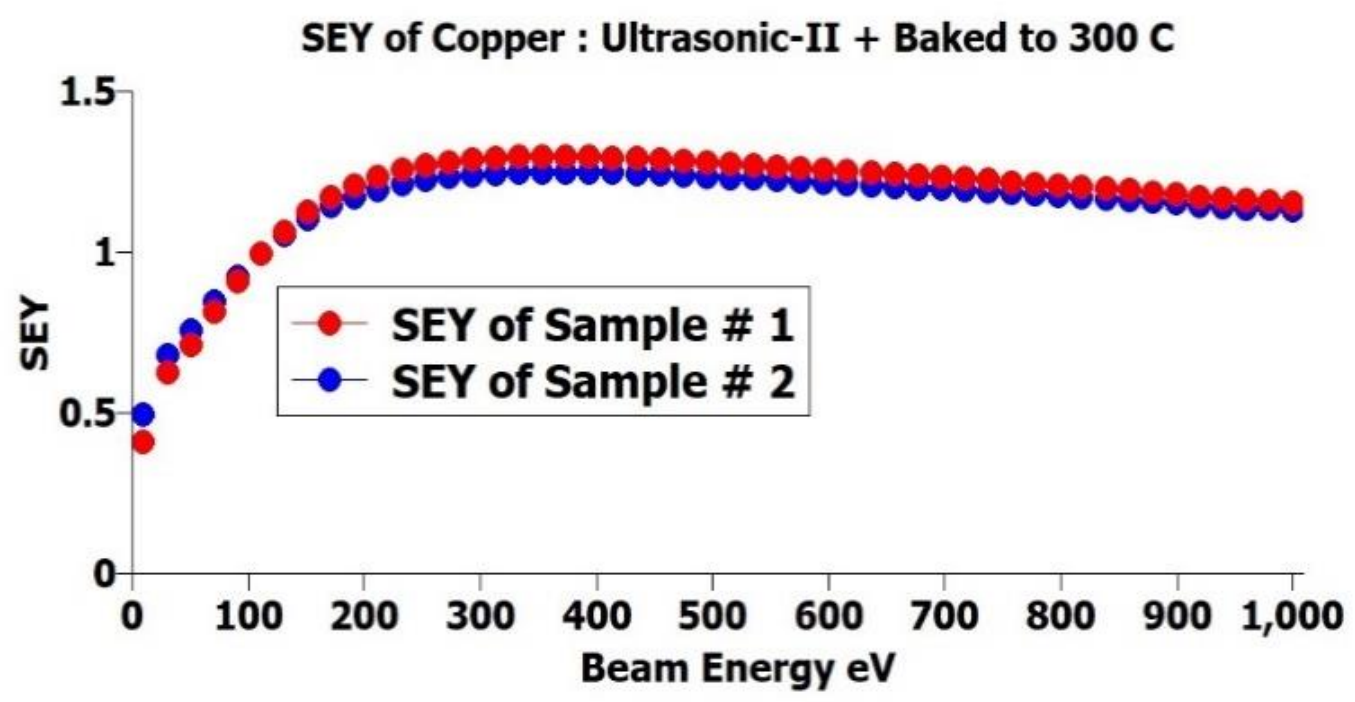

Figure 31: SEY OF ULTRASONIC-II CLEANED AND BAKED TO $300^{\circ} \mathrm{C}$ COPPER SAMPLES C WITH BEAM ENERGY SCANNED UP TO $1000 \mathrm{eV}$.

The $\delta_{\max }$ of sample \#1 and sample \#2 are 1.29 and 1.24, respectively, at corresponding $E_{\max }$ $\approx 350 \mathrm{eV}$ (a $4 \%$ variation), which gives some indication of repeatability. The $\delta_{\max }$ of sample \# 2 is the lowest peak value of copper's SEY reported in this thesis work. It is pertinent to mention here that these two samples were from the same batch.

Figure 32 shows the SEY for copper sample \#1 that was tested for the second crossover point by scanning the beam energy from $10 \mathrm{eV}$ to $1980 \mathrm{eV}$. Copper sample \#1 is the same sample tested in Fig. 31 (shown in red) for beam energy scanned from $10 \mathrm{eV}$ to $1000 \mathrm{eV}$. Copper sample \#2 was ex-situ cleaned with the Ultrasonic-II protocol and was in-situ baked 
in vacuum to $300^{\circ} \mathrm{C}$ for 3 hours. The $\delta_{\max }$ of the SEY curve shown in Fig. 32 is 1.28 at corresponding $\mathrm{E}_{\max } \approx 400 \mathrm{eV}$.

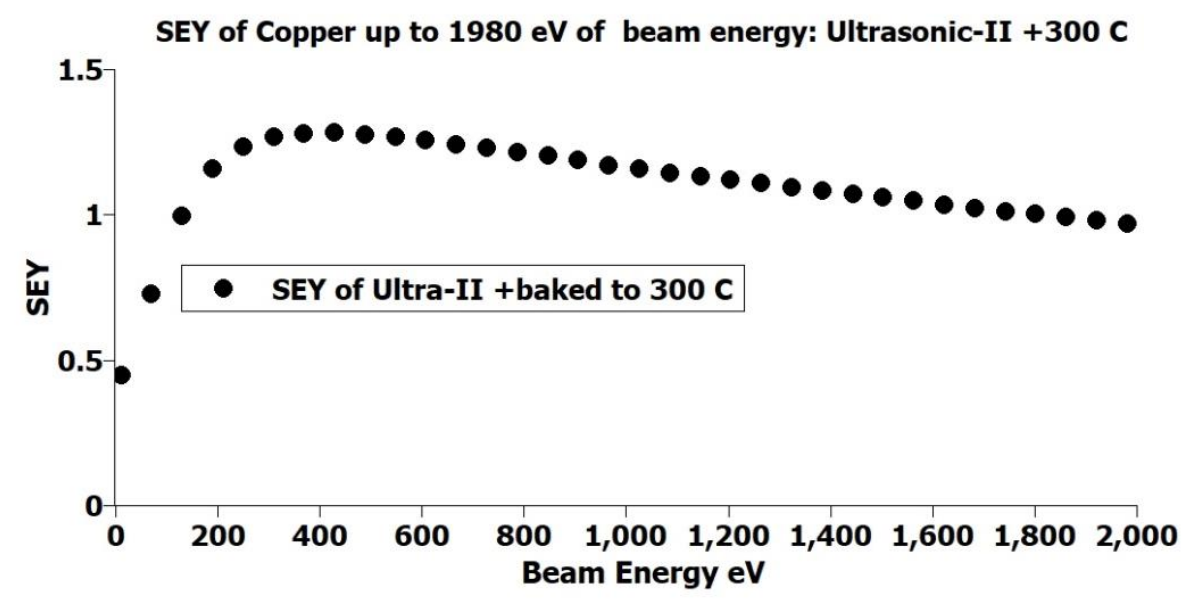

Figure 32: SEY OF ULTRASONIC-II CLEANED COPPER + BAKED TO $300^{\circ} \mathrm{C}$ WITH BEAM ENERGY SCANNED UP TO $1980 \mathrm{eV}$.

The second crossover point for Ultra-II + baked to $300^{\circ} \mathrm{C}$ copper is at $\mathrm{E}_{\mathrm{II}} \approx 1800 \mathrm{eV}$. The SEY resulting from this cleaning protocol is better (less/reduced) than the SEY generated from the Ultrasonic-I + baked to $300^{\circ} \mathrm{C}$ protocol. It is also evident from the second crossover point comparison of the Ultra-1 and Ultra-2 protocols i.e. $\mathrm{E}_{\mathrm{II}} \approx 1960 \mathrm{eV}$ for Ultrasonic-I (as shown in Fig. 30) and $\mathrm{E}_{\mathrm{II}} \approx 1800 \mathrm{eV}$ for Ultrasonic-II, that SEY performance of Ultrasonic-II protocol is better.

Figure 33 shows the influence of different surface treatments on the SEY of copper. It can be seen in the figure that the as-received sample showed the highest SEY, presumably due to presence of surface adsorbates and contaminants, whereas the sample cleaned with combination of ex-situ Ultra-II and baked to $300^{\circ} \mathrm{C}$ for 3 hours in vacuum resulted in the 
lowest peak SEY value and highest crossover energy $\mathrm{E}_{\mathrm{I}}$. It is pertinent to mention here that, except as-received samples, all other samples treated with cleaning protocols shown in Fig. 33 were from the same batch. The as-received copper showed the highest SEY while Ultra$\mathrm{II}+300^{\circ} \mathrm{C}$ showed the lowest SEY .

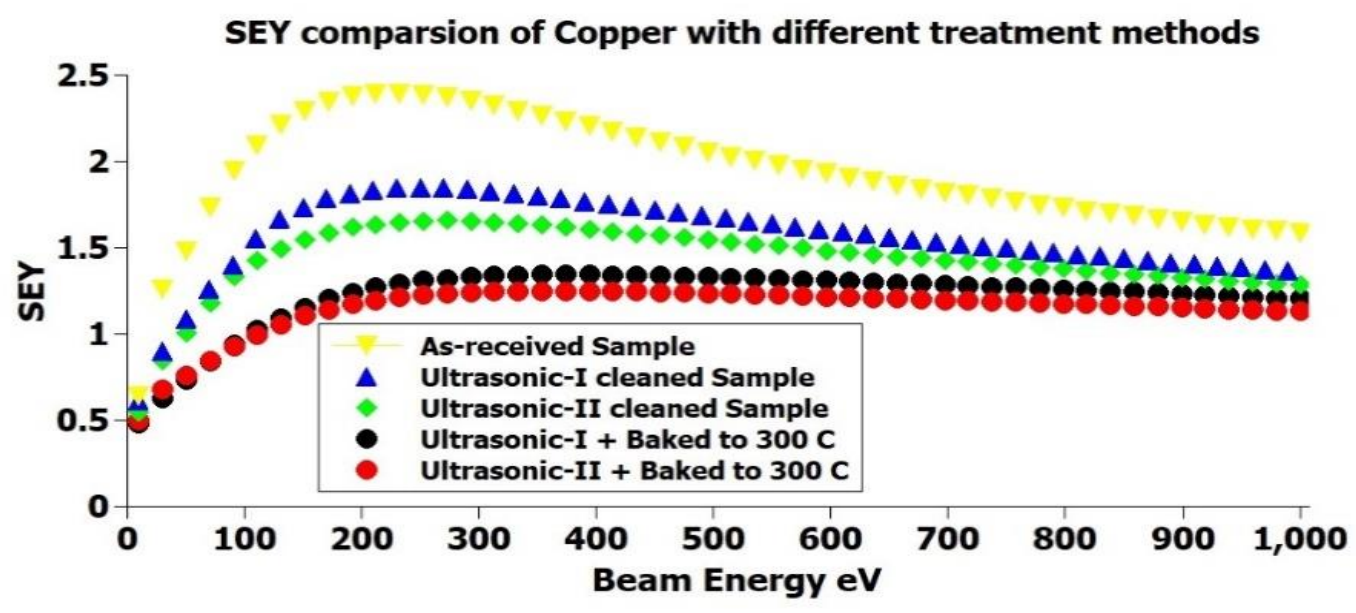

Figure 33: VARIATION IN SEY OF COPPER WITH DIFFERENT SURFACE TREATMENTS.

The analysis for Fig. 33 is as follows:

1) The SEY curve of as-received copper is represented by $\delta_{\max } \approx 2.4$ at a corresponding $\mathrm{E}_{\max } \sim 230 \mathrm{eV}$ beam energy. Clearly, cleaning the sample with both Ultrasonic-I and Ultrasonic-II protocols reduces the measured SEY of copper. Ultrasonic-II outperformed the Ultrasonic-I protocol.

2) Baking the Ultrasonic-I and Ultrasonic-II samples in vacuum reduces the difference of the SEY between samples cleaned by the two protocols. Ultrasonic$\mathrm{II}+$ baking to $300^{\circ} \mathrm{C}$ for 3 hours yielded the lowest SEY with $\delta_{\max } \approx 1.24$ at corresponding $\mathrm{E}_{\max } \sim 400 \mathrm{eV}$. It is also observed that as the SEY significantly decreases due to baking in vacuum, $E_{\max }$ and $E_{1}$ shift to higher primary beam 
energies. $\mathrm{E}_{1}$ shifts to $110 \mathrm{eV}$ and $130 \mathrm{eV}$ for Ultrasonic-1 + baking and UltrasonicII + baking respectively.

3) It is also observed that, as the surface is cleaned, the SEY curve tends to plateau as can be seen in the case for Ultra-I and Ultra-II + baking. The trend of reduced SEY with Ultrasonic I and Ultrasonic II + baked to $300^{\circ} \mathrm{C}$ seems to show good agreement with the SEY achieved through AGD (argon glow discharge) cleaning in the literature [25].

Figure 34 shows the influence of baking the Ultrasonic-II cleaned sample at different temperatures. An Ultrasonic-II cleaned sample was baked at $100^{\circ} \mathrm{C}, 200^{\circ} \mathrm{C}$, and $300^{\circ} \mathrm{C}$ in vacuum, respectively.

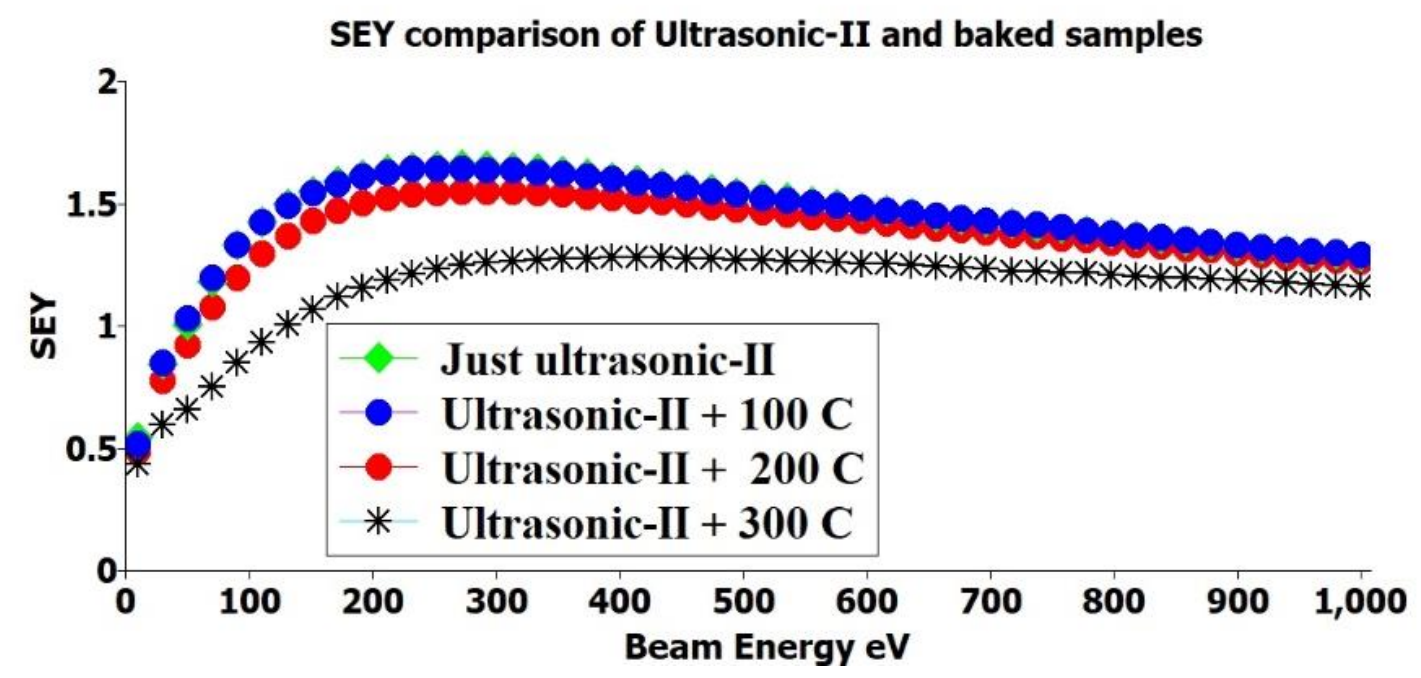

Figure 34: SEY COMPARISON OF ULTRASONIC-II CLEANED AND BAKED AT THE TEMPERATURES INDICATED.

The results presented in Fig. 34 show that increased heating helps to reduce the SEY at a temperature as high as $300^{\circ} \mathrm{C}$. It should be noted in Fig. 34 that SEY achieved through Ultrasonic-II cleaning alone is almost equivalent to SEY measured on the surface cleaned 
with a combination of Ultra-II and baking at $100^{\circ} \mathrm{C}$ and $200^{\circ} \mathrm{C}$. That is, baking to $100^{\circ}$ $\mathrm{C}$ and $200^{\circ} \mathrm{C}$ did not make a significant difference to the SEY. It is pertinent to mention here that SEY measurements were made as soon as the temperature reached the level of $100^{\circ} \mathrm{C}$ and $200^{\circ} \mathrm{C}$. Baking the sample up to $300^{\circ} \mathrm{C}$ for 3 hours yields the lowest SEY with $\delta_{\max } \approx 1.27$ at corresponding $\mathrm{E}_{\max } \approx 430 \mathrm{eV}$. This shows that baking at high temperature, i.e., $300^{\circ} \mathrm{C}$ for at least 3 hours, which presumably liberates contaminates from the sample surface, is most effective in lowering the SEY.

5) Variance in the SEY data

From the previous results, it appears that SEY is a surface-specific phenomenon and SEY varies significantly with surface treatments. In Fig. 35, the case of minimum variance in SEY data is illustrated. It should also be noted that the peak value of SEY $\left(\delta_{\max }\right)$ increased after baking was turned off. It may be that adsorbates liberated while baking the sample returned to the surface after the heater is turned off.

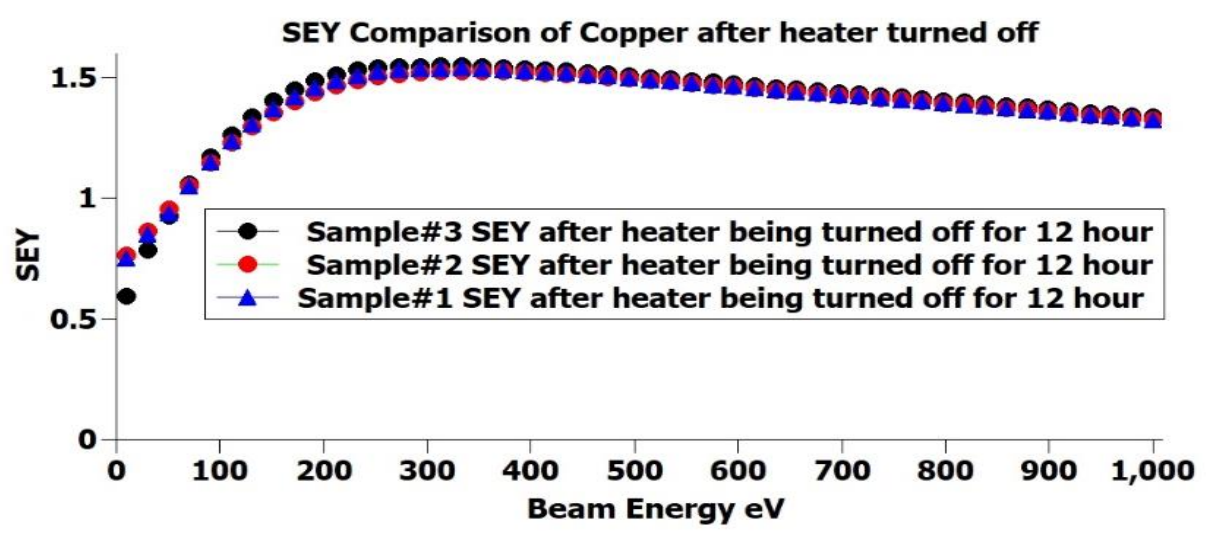

Figure 35: REPEATABILITY IN SEY DATA OF COPPER AFTER 12 HOURS OF HEATING BEING TURNED OFF. 
Three samples were tested after cleaning them with Ultrasonic-II and baking them to $300^{\circ}$ C. After reaching a temperature of $300^{\circ} \mathrm{C}$, baking was turned off for the next 12 hours and the temperature on the sample was observed to be around $27^{\circ} \mathrm{C}$. This was repeated for all three samples and SEY was recorded for each sample. It is observed that maximum repeatability is achieved with the SEY data measured on three different samples after the heating was turned off. The maximum repeatability in SEY data is achieved for three copper samples with $\delta_{\max }$ varying less than $1.5 \%$.

In order to investigate how SEY changes with time after heating is removed, SEY measurements were taken at one hour intervals, over a 6 hour period, after the heater was turned off. Figure 36 shows these measurements on a copper sample.

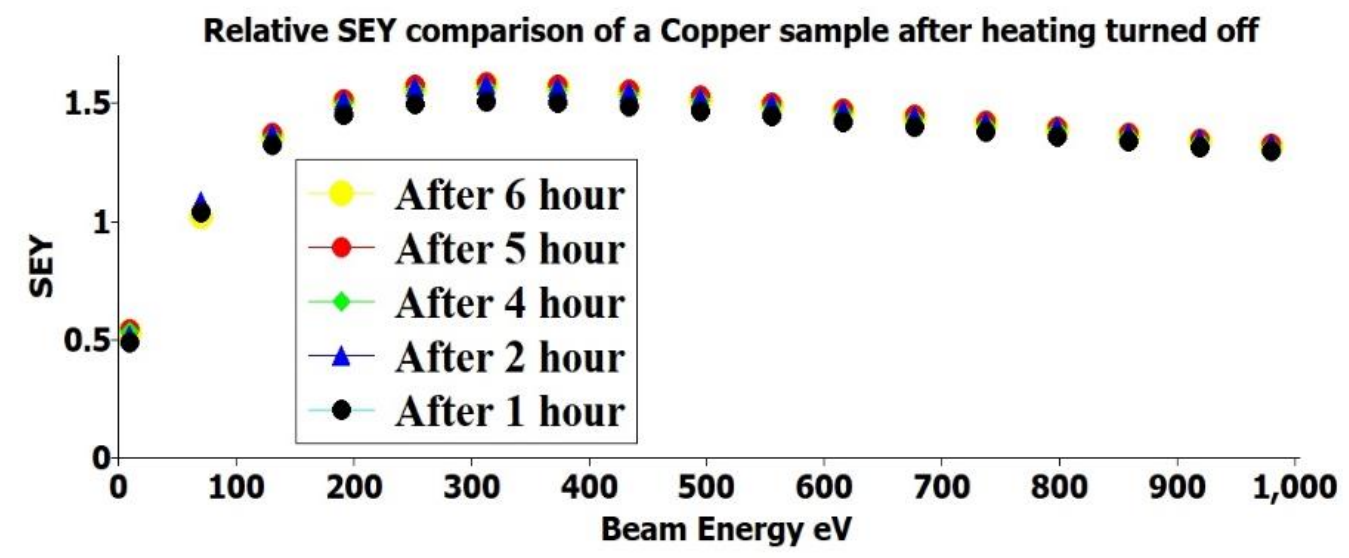

Figure 36: REPEATABILITY IN SEY DATA OF COPPER AFTER 1,2,3,4,5 AND 6 HOURS OF HEATING BEING TURNED OFF.

It can be seen that there was very little variability in the SEY curves after heating had be off for two hours. The peak SEY ( $\delta_{\max }$ ) variation for these measurements in under $5 \%$. 
6) As-received copper, heated

The effect of baking the as-received samples at different temperatures is demonstrated in Fig. 37. The copper sample was first baked to $100^{\circ} \mathrm{C}$ and the SEY was measured as shown by the green cuve. The sample was then baked to $200^{\circ} \mathrm{C}$ and the SEY was measured, shown by the red curve. Finally, the sample was baked to $300^{\circ} \mathrm{C}$ and the SEY was measured, shown by black curve. Baking at $100^{\circ} \mathrm{C}$ and $200^{\circ} \mathrm{C}$ was done for one hour while baking at $300^{\circ} \mathrm{C}$ was done for 3 hours.

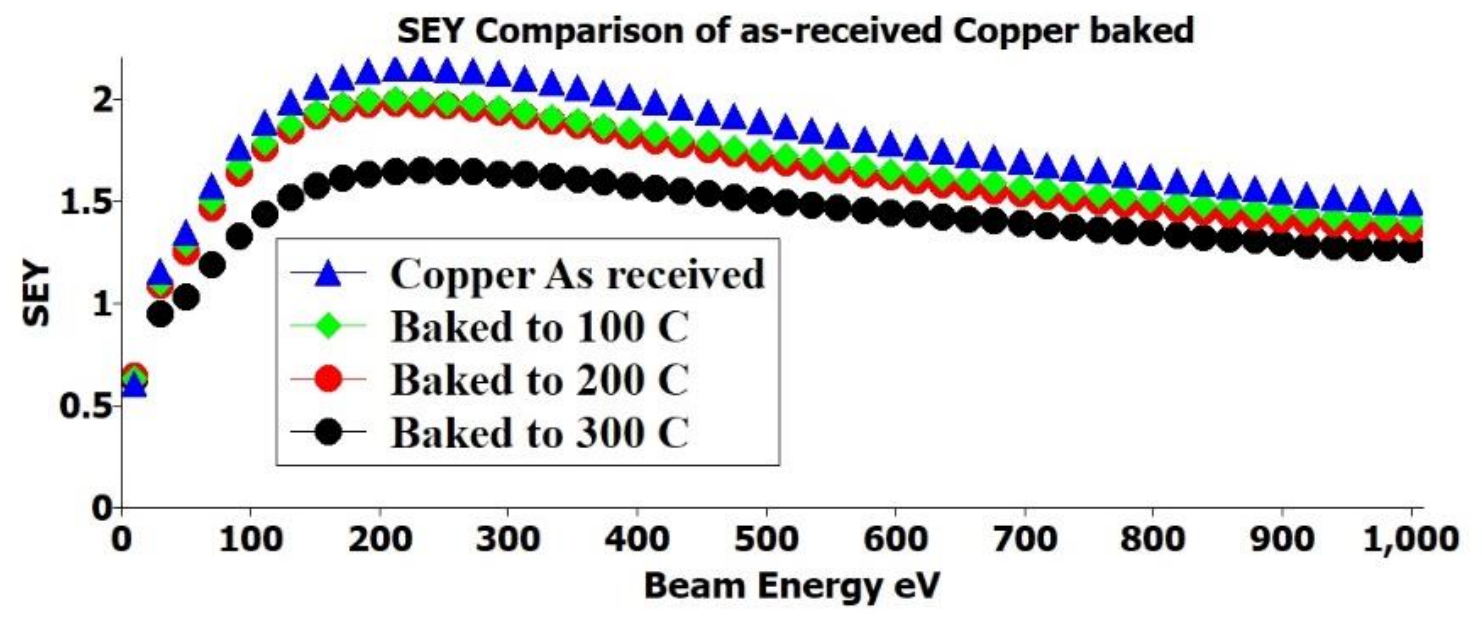

Figure 37: COMPARISON OF THE SEY OF AS-RECEIVED COPPER SAMPLE \# 1 BAKED AT VARIOUS TEMPERATURES.

The as-received copper, as expected, showed the highest SEY, while baking as-received to $300^{\circ} \mathrm{C}$ showed the lowest SEY. $\delta_{\max } \approx 2.15$ of as-received copper, while $\delta_{\max } \approx 1.65$ at corresponding beam energy $\approx 230 \mathrm{eV}$.

In order to check repeatability of baked as-received samples, copper sample \# 2 was tested in the same way with baking at $100^{\circ} \mathrm{C}, 200^{\circ} \mathrm{C}$, and $300^{\circ} \mathrm{C}$. Results are shown in Fig. 38 . It can be seen in the figure that the SEY of the as-received sample and the sample baked at 
$100^{\circ} \mathrm{C}$ overlap across the entire beam energy range. It is also known from Fig. 37 that the SEY of as-received and baked to $100^{\circ} \mathrm{C}$ samples did not show any significant differences. Baking sample \#2 to $200^{\circ} \mathrm{C}$ did not reduce the SEY considerably, which is also the case for sample \#1 shown in Fig. 37. Baking to $300^{\circ} \mathrm{C}$ reduces the SEY of sample \#2 significantly as compared to the SEY of the as-received sample.

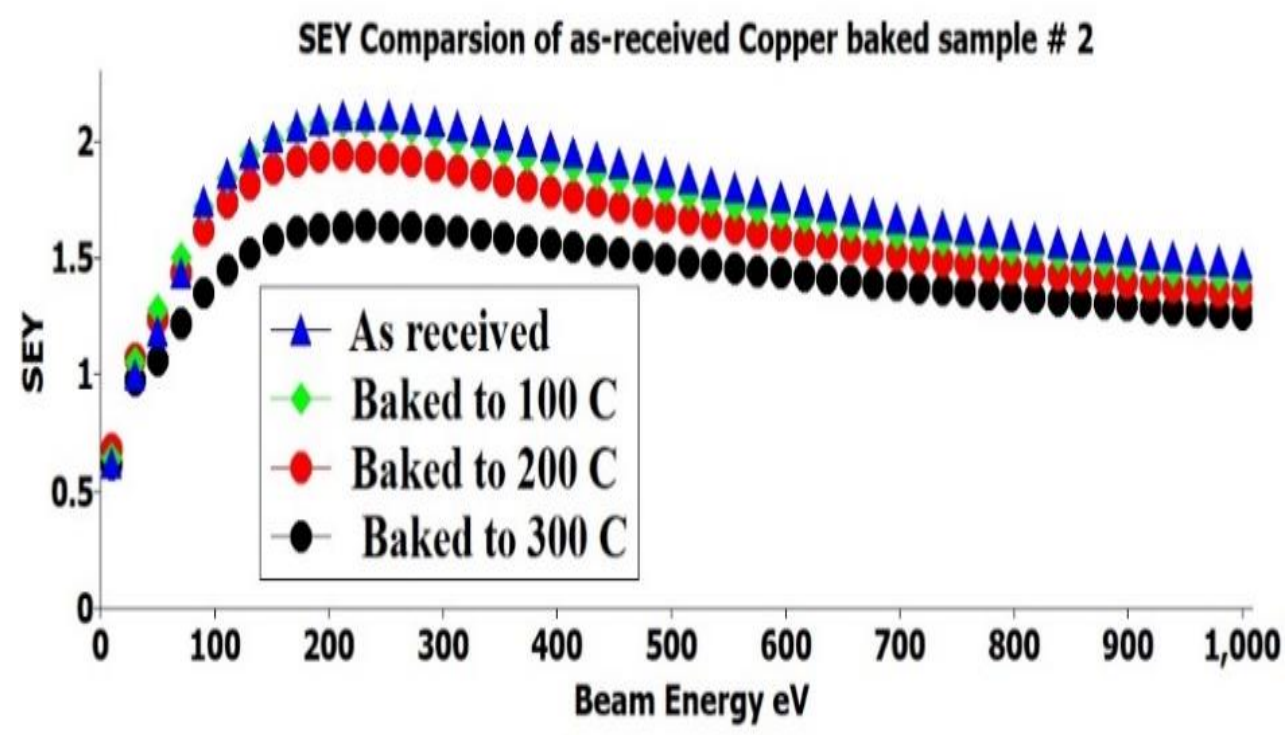

Figure 38: COMPARISON OF THE SEY OF AS-RECEIVED COPPER SAMPLE \# 2 BAKED AT VARIOUS TEMPERATURES.

It can be seen from the figure that the as-received copper sample showed the highest SEY with $\delta_{\max }=2.11$ at corresponding $\mathrm{E}_{\max } \approx 230 \mathrm{eV}$. The lowest SEY is obtained again by baking the sample to $300^{\circ} \mathrm{C}$ with $\delta_{\max }=1.63$ at corresponding $\mathrm{E}_{\max } \approx 230 \mathrm{eV}$. Although the surface treatment for sample \#2 was the same as for sample \#1, the SEY results for both samples are not equivalent. This supports the notion that SEY is a surface-sensitive phenomenon and repeatability is difficult to achieve, even using the same protocol. It should also be noted that as-received copper sample $\# 1$ and sample $\# 2$ baked at $100^{\circ} \mathrm{C}$ and $200^{\circ} \mathrm{C}$ shown in Figs. 37 and 38, respectively, were heated for one hour at specified 
temperatures. Presumably, baking the sample at $100^{\circ} \mathrm{C}$ or $200^{\circ} \mathrm{C}$ for longer period of time, i.e., at least 3 hours, might have resulted in considerably reduced SEY.

7) SEY of air-exposed copper

Figure 39 plots the SEY of air-exposed copper and baked copper.

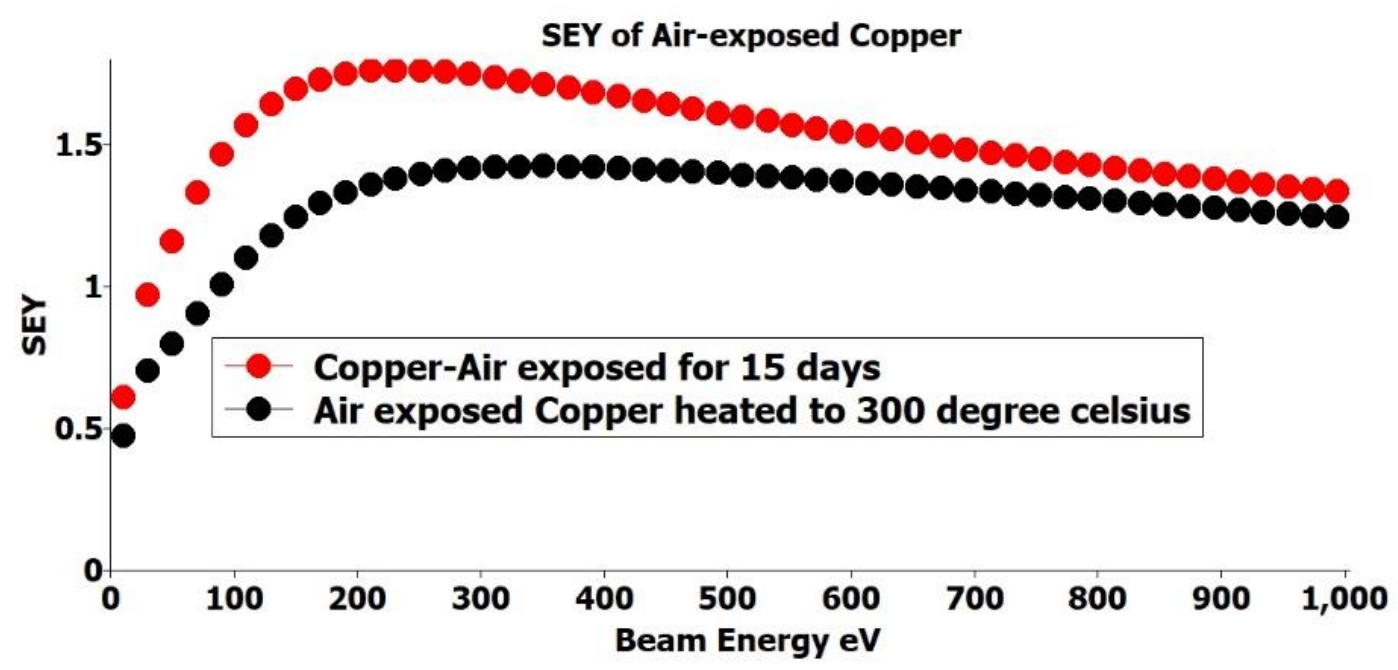

Figure 39: SEY COMPARISON OF AIR EXPOSED COPPER WITH AND WITHOUT BAKING.

The copper sample of Fig. 39 was exposed to air for 15 days at room temperature. It is apparent that the SEY of air-exposed copper is significantly reduced as compared to asreceived copper. It has been reported previously that the metal oxide of copper has lower SEY than its parent metal [43]. The decrease in SEY due to air exposure is therefore likely due to the formation of a copper oxide layer on the material surface. The same air - exposed copper was baked to $300^{\circ} \mathrm{C}$ for 3 hours in vacuum, which resulted in further decrease in SEY shown by the black curve in Fig. 39.

An attempt to enhance the formation of copper oxide on the surface was made by baking the copper in air. Figure 40 shows the effect of baking the copper in air followed by baking 
in vacuum. Baking the sample in air refers to the procedure that it was baked in the vacuum chamber while the chamber was at atmospheric pressure. After baking at the specified temperature at atmospheric pressure, the pumps were turned on to bring the pressure to the UHV region. The SEY measurement was made as soon as the vacuum pressure stabilized (black curve in Fig. 40). The sample was then again baked to $300^{\circ} \mathrm{C}$ for 6 hours in vacuum and SEY measurements were repeated (red curve).

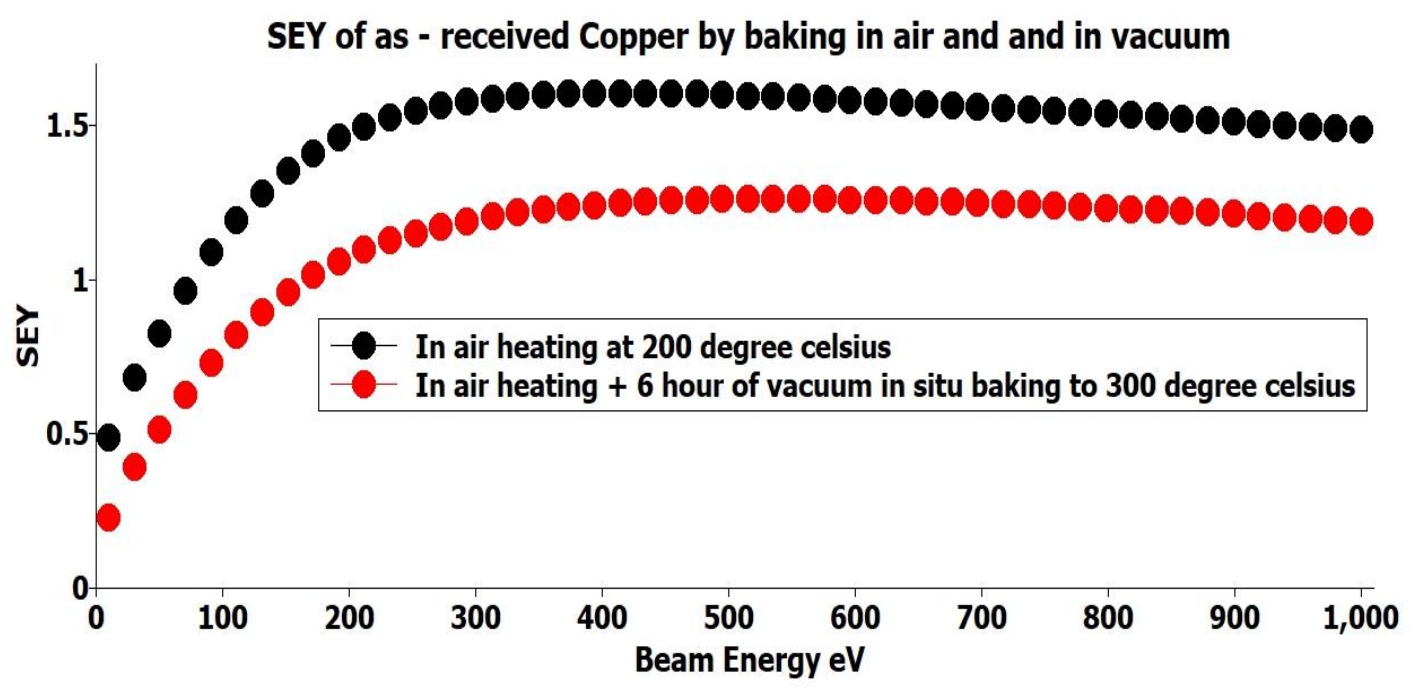

Figure 40: COMPARISON OF AS-RECEIVED COPPER BAKED IN AIR AND IN VACUUM.

The as-received copper sample was first baked in air up to $200^{\circ} \mathrm{C}$ and then baked in vacuum for 6 hours at $300^{\circ} \mathrm{C}$. Baking to $200^{\circ} \mathrm{C}$ in air resulted in an $\delta_{\max } \approx 1.60$ at about $400 \mathrm{eV}$ of beam energy, while heating the air-baked sample to $300^{\circ} \mathrm{C}$ for 6 hours in vacuum decreased the $\delta_{\max }$ to 1.26 with shifted $\mathrm{E}_{\max }$ at $\approx 515 \mathrm{eV}$. The $\mathrm{E}_{1}$ point corresponding to black and red curve is at $90 \mathrm{eV}$ and $150 \mathrm{eV}$ respectively. This shift of $\mathrm{E}_{\max }$ and $\mathrm{E}_{1}$ to higher electron beam energies is typical for clean surfaces. It has been shown before that $E_{\max }$ and $E_{1}$ is at lower beam energies for contaminated (as-received) surfaces. 
The heating of copper to $200^{\circ} \mathrm{C}$ in air caused roughness on the surface [43]. It has been reported that rough surfaces release fewer secondary electrons when compared to smooth surfaces [22]. In addition, heating copper in air causes oxidation of copper by forming a layer of copper oxide on the surface. Most metal oxides have high SEY, but copper oxide, which is a semiconductor, has a lower SEY than its parent metal [22][43].

\subsubsection{SEY of Silver}

This section covers the SEY of a silver sample by applying different surface treatment methods. Results show that the SEY of silver varies with different cleaning protocols and, further, the effect of dose is illustrated on the SEY of silver. Silver sample of purity level $99.99+\%$ and part number AG000480 was procured from Good Fellow USA for all SEY measurements that will follow.

1) Ultrasonic-1 cleaned silver

A silver sample cleaned using the Ultrasonic-1 cleaning protocol was subjected to 10 shots where each shot represents the SEY curve. The dose for each shot was maintained around $10^{-6} \mathrm{C} / \mathrm{mm}^{2}$ to avoid coupling beam conditioning to the SEY measurements. Figure 41 shows the SEY of Ultrasonic-I silver for 10 shots. The SEY variation for these measurements is under $2 \%$. The peak value $\left(\delta_{\max }\right)$ of the silver's SEY is lower than the peak value of copper's SEY cleaned with same protocol (Ultrasonic-I) for all 10 shots. The first crossover beam energy $\left(\mathrm{E}_{1}\right)$ point for the silver sample is at $40 \mathrm{eV}$ while $\mathrm{E}_{1}$ point for the copper sample is at $30 \mathrm{eV}$. The plot of 10 shots of copper's SEY is shown in Fig. 22. Presumably, the peak value reduction of the SEY of silver sample could be due to the higher dose received by it as the electron beam energy was scanned up to $1700 \mathrm{eV}$. 


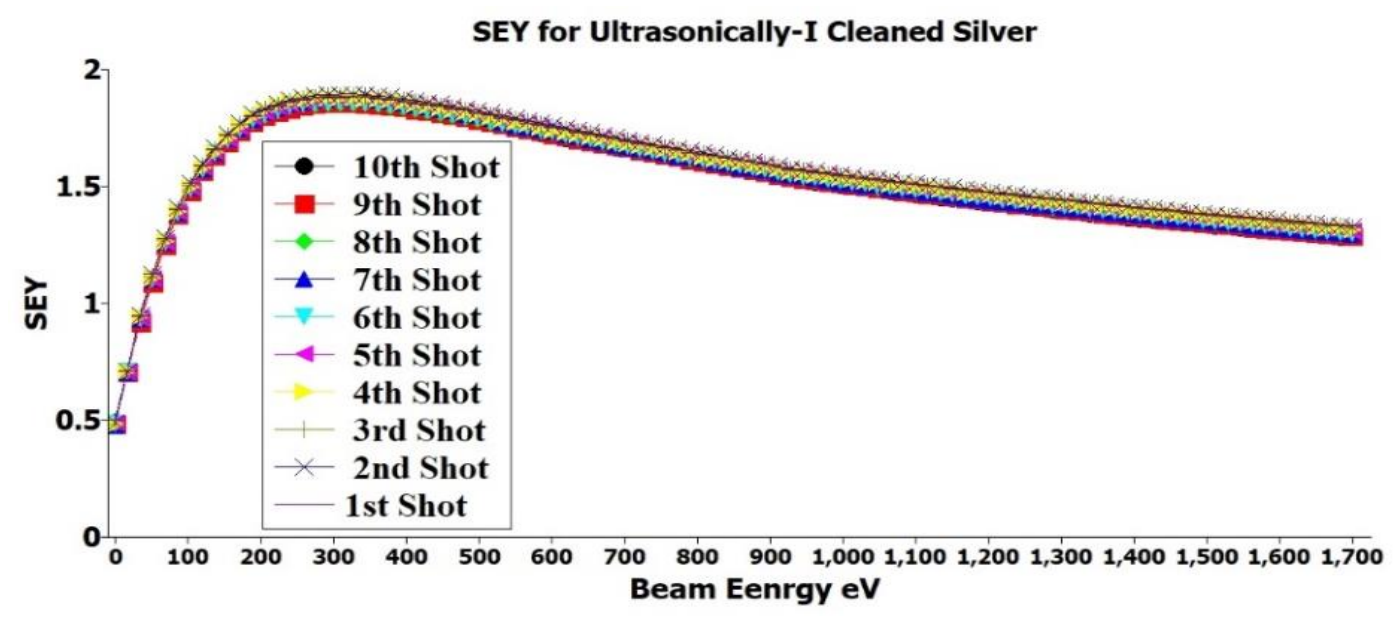

Figure 41: 10 SHOTS OF SEY OF ULTRASONIC-I CLEANED SILVER.

The SEY was measured while scanning the beam energy from $10 \mathrm{eV}$ to $1700 \mathrm{eV}$. $\delta_{\max }$ for the first and last shot is 1.88 and 1.85 , respectively, at corresponding $\mathrm{E}_{\max } \approx 300 \mathrm{eV}$. SEY curves show little variation because the dose is kept minimum and constant for all shots.

Figure 42 shows the SEY of silver sample \#2 with Ultrasonic-I treatment. The peak value of SEY $\left(\delta_{\max }\right)$ has increased little as compared to measured SEY shown in Fig. 41.

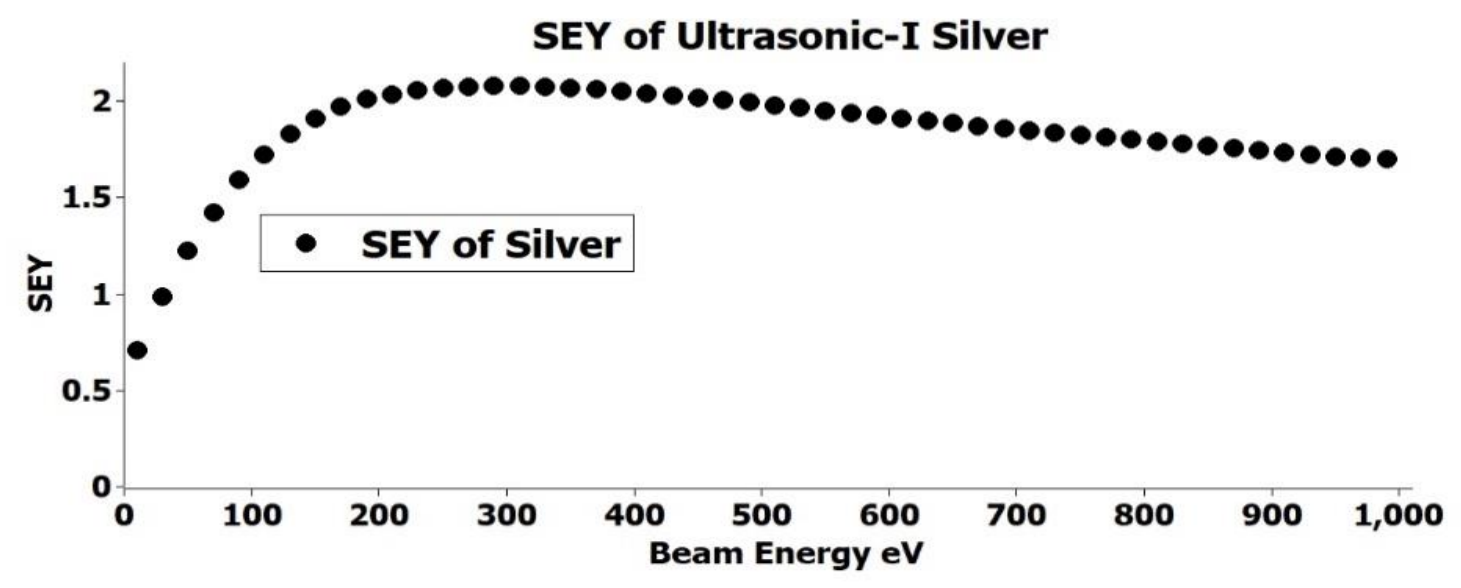

Figure 42: SEY OF ULTRASONIC - I CLEANED SILVER SAMPLE \# 2. 
The motivation to test a different sample (sample \#2) with the same Ultrasonic-I treatment is to check for repeatability. For silver sample \#2, the beam energy was scanned from 10 $\mathrm{eV}$ to $1000 \mathrm{eV}$ with the $\delta_{\max }=2.07$ at corresponding $\mathrm{E}_{\max } \approx 330 \mathrm{eV}$. $\delta_{\max }$ of sample \#2 is a little higher than that of sample \#1, which again indicates that SEY is a surface-sensitive process and there is significant variation in SEY, even when the same cleaning protocol is used.

Dose effect is important in SEY measurements as shown previously. Subjecting the sample to a higher dose resulted in a decrease in SEY. The cumulative nature of the dose-effect was tested on a silver sample and is discussed in the following section.

\section{2) Dose Effect}

When the surface of a sample is exposed to the impact of electron beam, its SEY as a function of electron dose decreases. Results show that SEY at any beam energy is dependent on the total charge received by the sample surface up to that point.

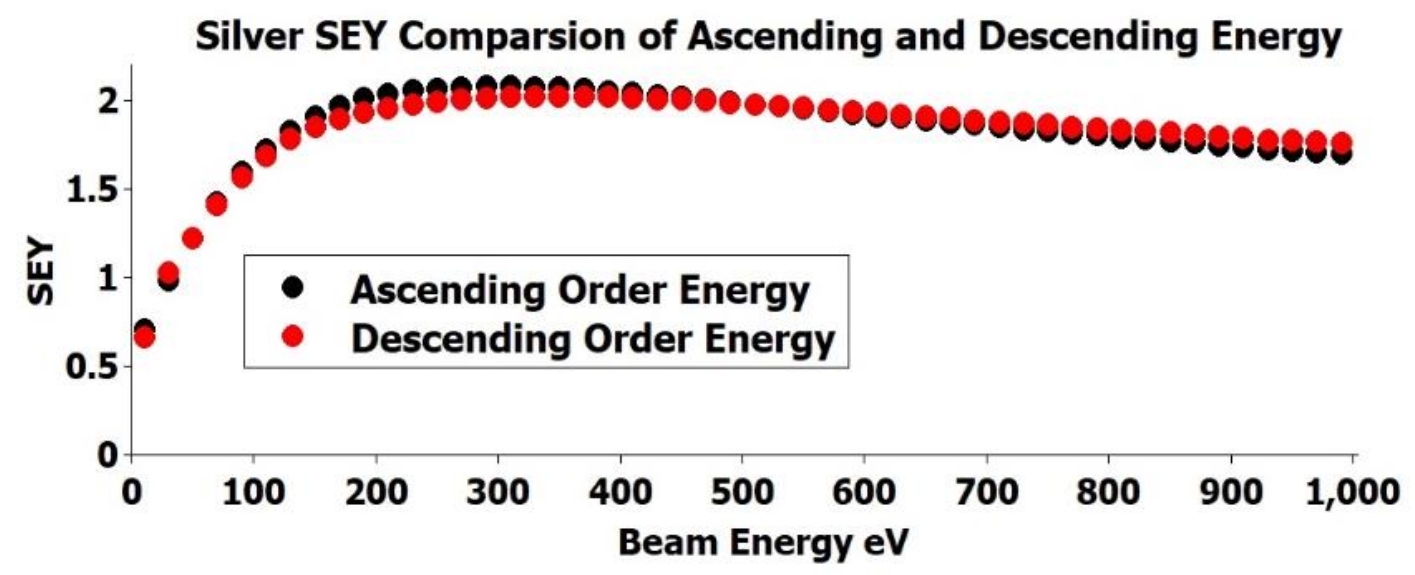

Figure 43: SEY COMPARISON OF SILVER SAMPLE WITH ASCENDING AND DESCENDING BEAM ENERGY. 
Figure 43 shows a plot of a SEY for a silver sample first bombarded with an electron beam as it is scanned from low to high beam energy, and then scanned from high to low beam energy. The result of the cumulative nature of the dose-effect agrees with the theory and existing literature [21]. For descending beam energies, less charge is received by the sample at higher energies compared to lower energies, while in ascending order the converse is true. So, for descending beam energy, lower energies will show a lower SEY, while higher energies would have a higher SEY compared to the ascending scan experiment.

3) Comparison of different cleaning protocols

SEY measured against different cleaning protocols for silver is shown in Fig. 44.

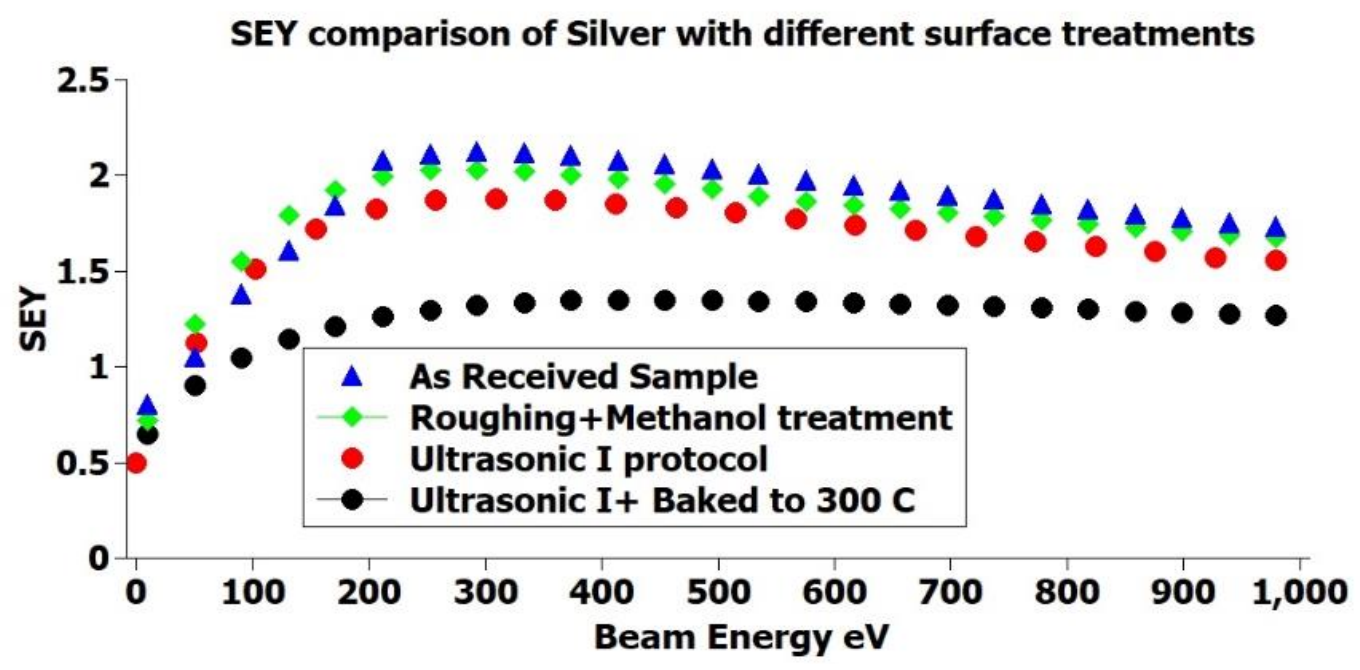

Figure 44: VARIATION IN SEY OF SILVER WITH DIFFERENT SURFACE TREATMENTS.

The as-received sample, as expected, showed the highest SEY with $\delta_{\max }=2.12$ at corresponding $\mathrm{E}_{\max } \approx 300 \mathrm{eV}$. The Ultrasonic-I cleaning reduced the $\delta_{\max }$ to 1.88 at corresponding $\mathrm{E}_{\max } \approx 330 \mathrm{eV}$, while baking the sample Ultrasonic-I sample to $300^{\circ} \mathrm{C}$ 
significantly reduced the SEY with $\delta_{\max }=1.35$ at $\mathrm{E}_{\max }=450 \mathrm{eV}$. Roughing and cleaning with methanol did not significantly reduce the SEY.

4) Effect of cleaning protocols on the SEY of silver

Figure 45 shows a comparison of SEY resulting after treating the sample of silver with different surface treatments involving baking in vacuum. Tested samples are from the same batch. Two of the samples were cleaned ex-situ with Ultrasonic-I and Ultrasonic-II treatments and then baked for 3 hours to $300^{\circ} \mathrm{C}$ in vacuum respectively. One of the samples was installed into the vacuum chamber without any surface treatment, i.e., the as-received sample. The SEY of the as-received sample is higher, as expected, with $\delta_{\max } \approx 2.12$ at beam energy $\approx 300 \mathrm{eV}$. The SEY of Ultra-I,II + baked to $300^{\circ} \mathrm{C}$ treated silver is almost equivalent.

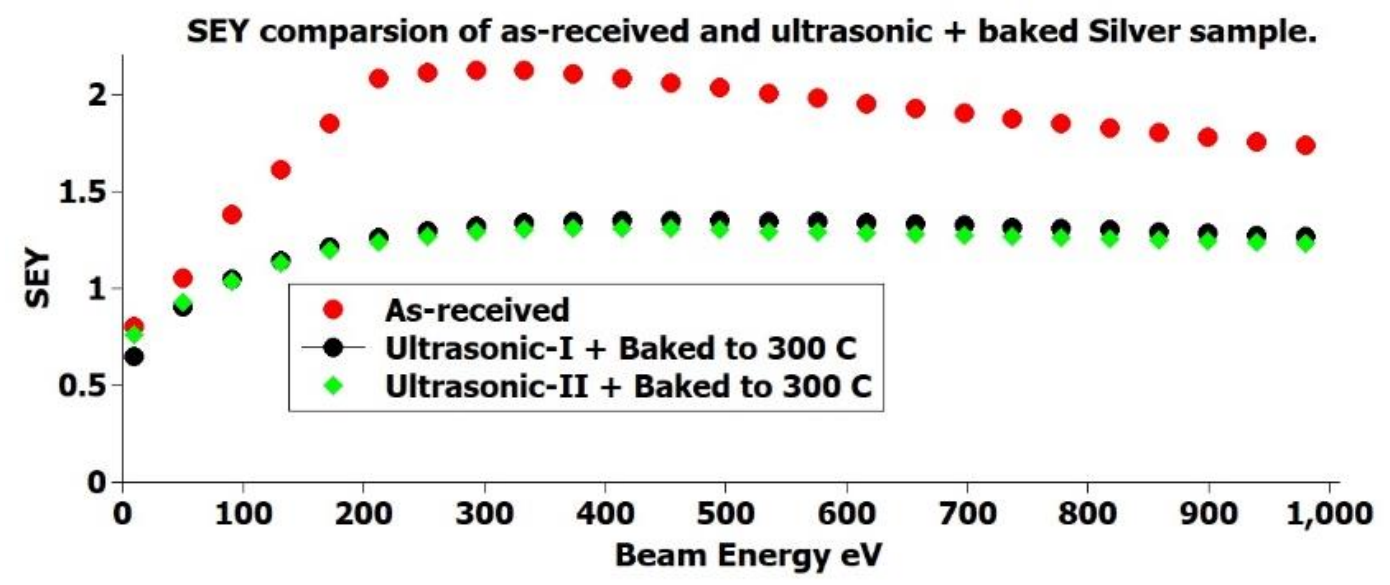

Figure 45: COMPARISON OF AS-RECEIVED AND ULTRASONIC-I, II + BAKED SILVER SAMPLES.

The Ultrasonic-II + baking to $300^{\circ} \mathrm{C}$ yielded the lowest SEY. $\delta_{\max }$ for the copper sample was $\approx 1.24$ at a corresponding $\mathrm{E}_{\max } \approx 400 \mathrm{eV} . \delta_{\max }$ for the silver sample was $\approx 1.30$ at a corresponding $\mathrm{E}_{\max } \approx 400 \mathrm{eV}$. The SEY trend of the Ultrasonic1 + baked to $300^{\circ} \mathrm{C}$ and the 
Ultrasonic II+ baked to $300^{\circ} \mathrm{C}$ protocols seem to show good agreement with the SEY achieved through AGD (Argon glow discharge) cleaning from previous research studies [25].

\subsubsection{SEY comparison of Ultrasonic-I cleaned alloys}

So far in this thesis, the SEY measurements performed on pure single metals, i.e., copper and silver, have been shown. Figure 46 shows a comparison of the SEY of several alloys procured from Good Fellow USA, including aluminum 6061 (Purity: Al97.5/Mg 1/Si 0.6/Fe 0.5/Cu 0.4, part \# : AB190352) monel (Purity: Ni 65/ Cu 33/Fe 2, part \#: NI110550), invar (Purity: Fe 64/ Ni 36, part \#: FE023150), and nickel-cobalt (Purity: Fe 54/ Ni29/ Co17, part \# :FE040380). All alloys were cleaned using the Ultrasonic-I protocol. The following are the observations related to SEY measurements shown in Fig. 46.

1) It is evident from Fig. 46 that the SEY of the aluminum 6061 alloy is the highest and SEY of a nickel-cobalt alloy is the lowest. The peak value $\left(\delta_{\max }\right)$ of aluminum is 3.3, which is the largest SEY reported in this thesis work. The first crossover point $\left(\mathrm{E}_{1}\right)$ is at low energy, i.e., $\approx 20 \mathrm{eV}$, which is not desired in order to prevent multipactor breakdown. The reported peak SEY value of the as-received aluminum 6061 sample is around 3.5 [37]. Cleaning the aluminum 6061 with Ultrasonic-I seems to have not make any significant reducation in SEY as compared to reported SEY of as-received aluminum sample.

2) The SEY curves of invar and monel alloys overlap for most of the beam energy spectrum. $\delta_{\max }$ of the invar alloy is at a corresponding electron beam energy of 250 $\mathrm{eV}$ while $\delta_{\max }$ of monel is shifted at a corresponding electron beam energy of $\mathrm{E}_{\max }$ 
$\approx 310 \mathrm{eV}$. The $\mathrm{E}_{1}$ point for invar and monel is located at $\approx 20 \mathrm{eV}$ and $\approx 30 \mathrm{eV}$, respectively.

3) It is clear from the figure that the alloys tested show higher SEY than pure metals treated with the Ultra-I cleaning method. The Nickel-Cobalt alloy has shown the lowest $\mathrm{SEY}$ with $\mathrm{E}_{1}$ point located at $\approx 30 \mathrm{eV}$.

Keeping in view the large $\mathrm{SEY}$ and $\mathrm{E}_{1}$ point located at low beam electron energies, these alloys were not subjected to any other surface treatment methods except invar that was tested for roughed + methanol cleaning protocol. The measured SEY of invar by applying the roughed + methanol protocol is shown in Fig. 47 .

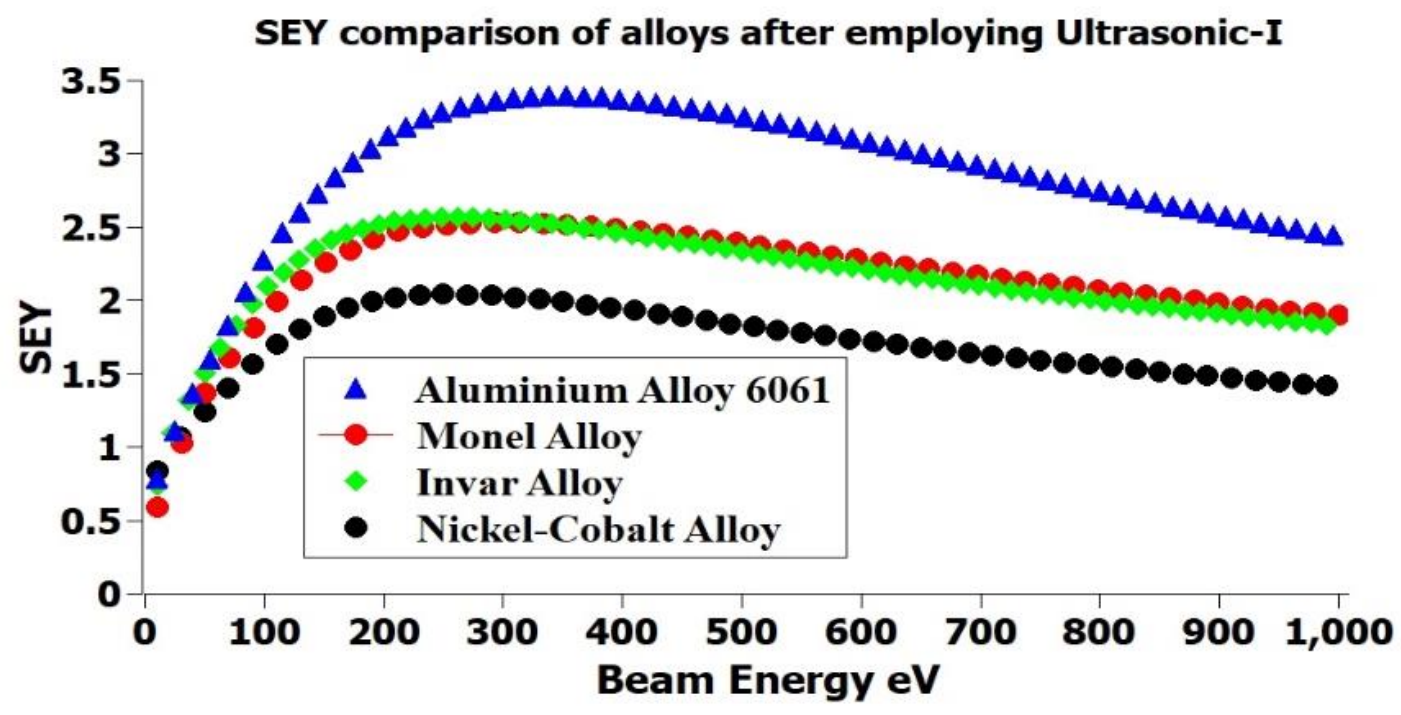

Figure 46: SEY COMPARISON OF ALLOYS WITH ULTRASONIC-I CLEANING.

$\delta_{\max }$ and $\mathrm{E}_{\max }$ for the alloys are also summarized in Table 7. 
Table 7: Parameters for the alloys.

\begin{tabular}{|c|c|c|c|}
\hline Sample & $\delta_{\max }$ & $\mathrm{E}_{\max }(\mathrm{eV})$ & $\mathrm{E}_{1}(\mathrm{eV})$ \\
\hline Aluminum 6061 & 3.3 & $\approx 350$ & 20 \\
\hline Monel & 2.53 & $\approx 310$ & 20 \\
\hline Invar & 2.56 & $\approx 250$ & 30 \\
\hline Nickel-cobalt & 2.04 & $\approx 250$ & 30 \\
\hline
\end{tabular}

\subsubsection{Comparison of ultrasonic-I and roughed + methanol}

The SEY results of Ultrasonic-I treated samples were shown in the preceding section.

Figures 47 and 48 show a comparison of SEY for invar, silver, copper, and stainless steel samples.

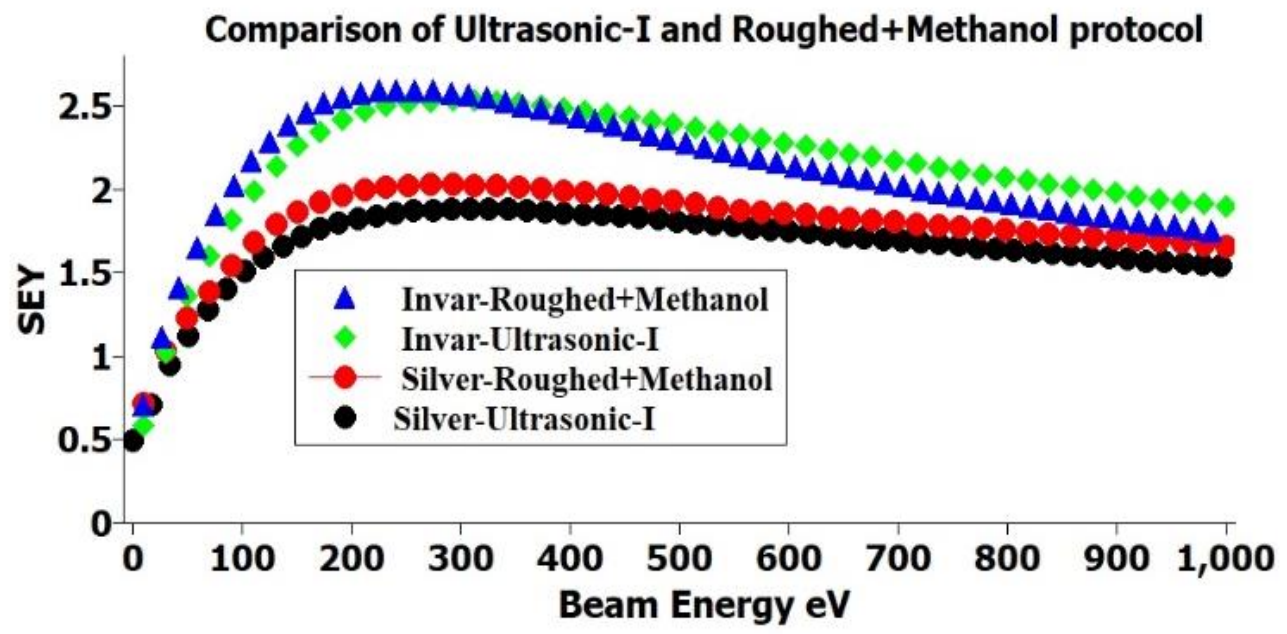

Figure 47: COMPARISON OF THE ULTRASONIC-I AND ROUGHED+METHANOL CLEANED INVAR AND SILVER SAMPLES. 
Surface characteristics play an important role in secondary electron emission. Surface roughness is one way to reduce SEY. This indirectly causes a reduction in the emissivity of the surface, as a solid angle for electrons to escape without further interaction with the vacuum chamber can be significantly decreased [37].

When secondary electrons are emitted from a rough surface, they are likely to be intercepted by adjacent surface irregularities such that they can deflect back to the surface. However, the electrons emitted from a smooth surface do not experience any such hurdles and escape from the surface more easily. Figure 47 shows that SEY of invar resulting from roughed + methanol protocol, which is equivalent to SEY achieved through the UltrasonicI protocol up to $400 \mathrm{eV}$ of energy while $\mathrm{SEY}$ is comparatively lower at high energies for the first protocol. The SEY of silver with both applied protocols, i.e., roughed + methanol and ultrasonic-I, is almost equivalent over the entire beam energy spectrum.

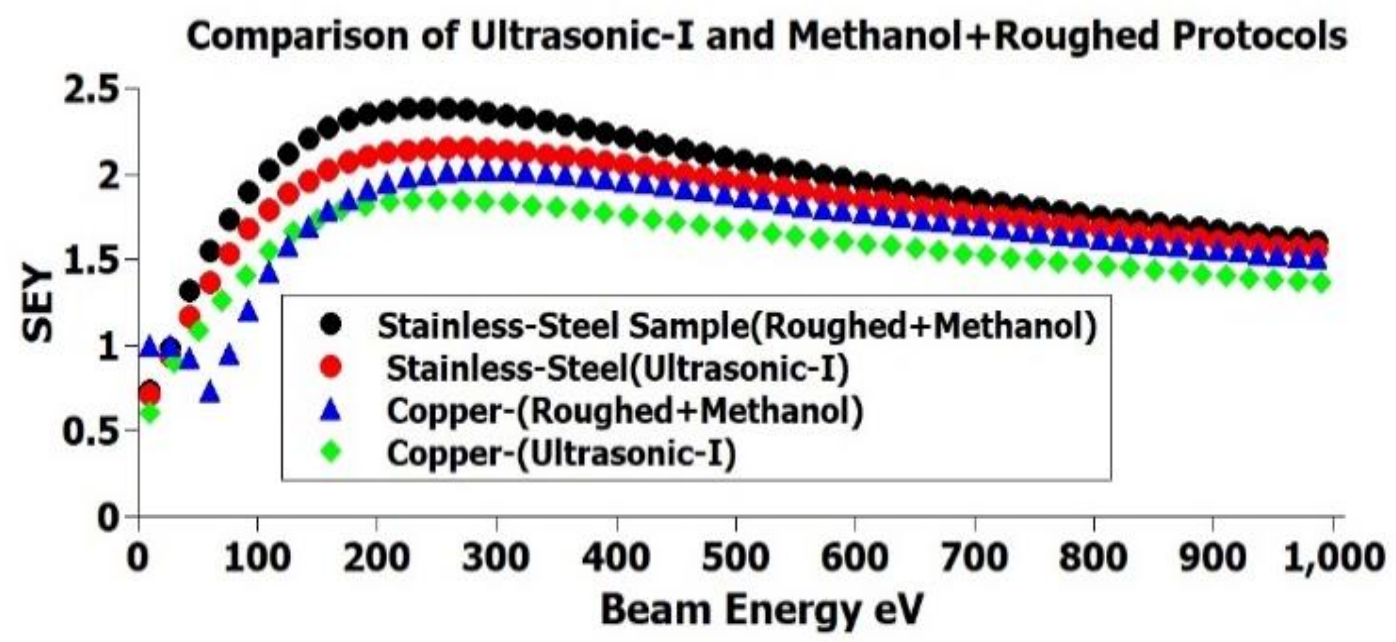

Figure 48: COMPARISON OF ULTRASONIC-I AND ROUGHED+METHANOL CLEANED STAINLESS STEEL AND COPPER SAMPLES. 
It can be seen in Figs. 47 and 48 that the SEY of the samples cleaned by the roughed + methanol protocol is higher than that of samples where the Ultrasonic-I protocol is employed for all materials under study. However, the use of the roughed + methanol protocol yields a lower SEY than as-received samples, such as copper and silver (See Fig. 21 and Fig. 45 as reference). It can be inferred from these results that copper cleaned with the Ultrasonic-I protocol has produced the minimum SEY.

\subsubsection{SEY of Aluminum 6061}

The SEY of aluminum 6061 alloy was also measured after applying different surface treatments, as shown in the following sections.

1) Aluminum exposed to air

The aluminum 6061 samples received from the vendor were kept in a bag for around 9 months before subjecting them to SEY measurement. The as-received aluminum sample was tested only for the ultrasonic-I protocol. Figure 49 shows the results of the SEY of two aluminum samples exposed to air for as long as about 9 months.

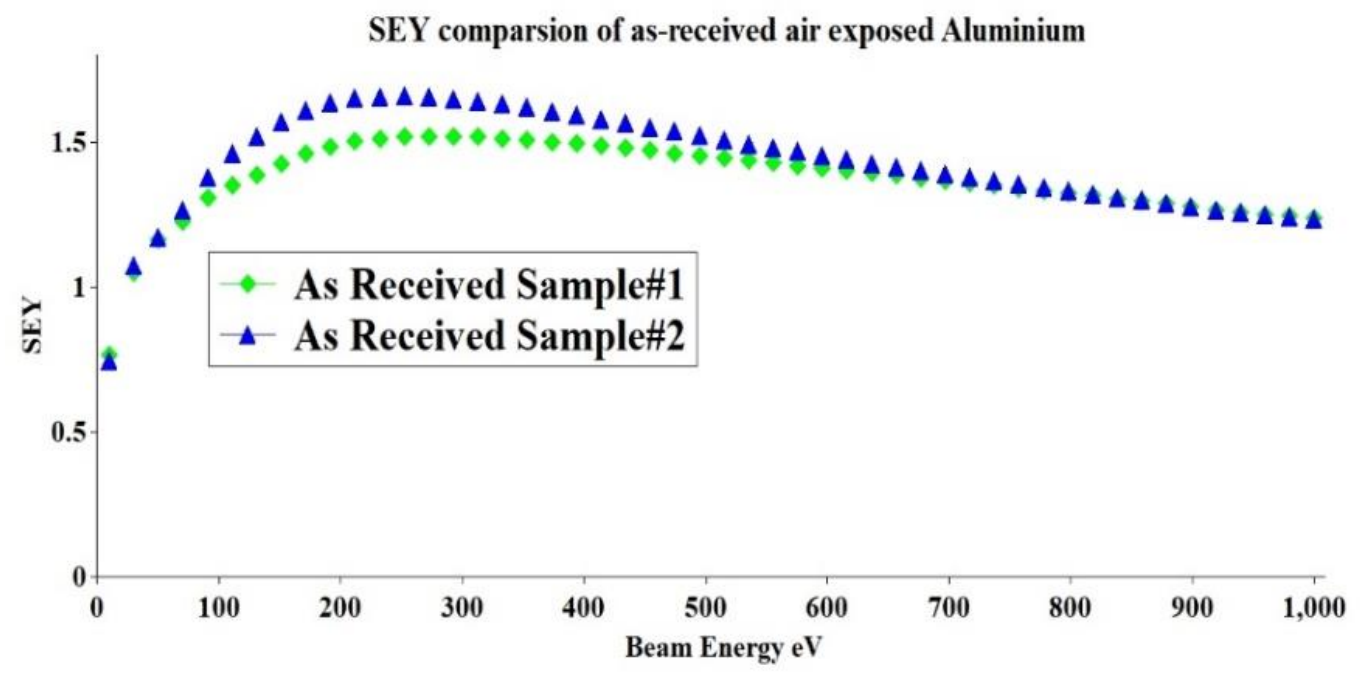

Figure 49: SEY COMPARISON OF AS-RECEIVED ALUMINIUM SAMPLES. 
It is important to note here that as-received samples were not fresh and were exposed to air for a long time, i.e., roughly 9 months. Two as-received samples were tested and $\delta_{\max }=$ 1.52 for sample $\# 1$ and $\delta_{\max }=1.65$ for sample \#2 at corresponding $\mathrm{E}_{\max } \approx 250 \mathrm{eV}$. The asreceived SEY of aluminum is usually high as aluminum is perceived as a high SEY material. The published data shows the SEY of as-received aluminum has $\delta_{\max }$ around 3.5. The SEY shown in Fig. 49 depicts significant reduction, presumably due to the formation of an oxide layer on the surface which occurred from exposure to air.

2) Baked As-received aluminum

Figure 50 shows the SEY of an as-received air-exposed aluminum 6061 sample baked to $300^{\circ} \mathrm{C}$ for 3 hours in vacuum. The aluminum sample used for this experiment was the same as the one that was exposed to air for about 9 months and its SEY is shown in Fig. 49.

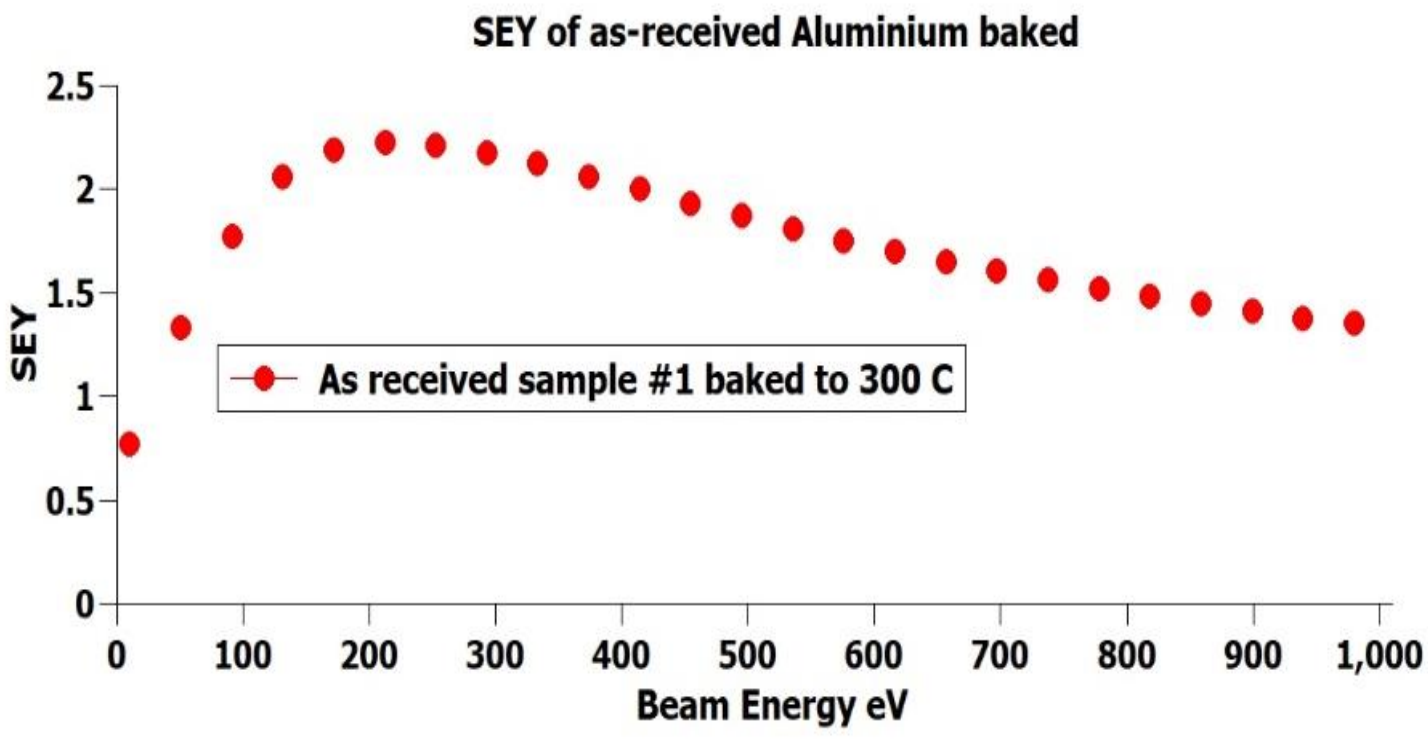

Figure 50: SEY OF AS-RECEIVED ALUMINIUM SAMPLE BAKED TO $300^{\circ} \mathrm{C}$. 
It has been shown so far that baking has reduced the SEY in all cases. In the case of asreceived aluminum exposed to air, baking increased the SEY instead of decreasing it. Baking the as-received sample increased $\delta_{\max }$ to 2.21 at a corresponding $\mathrm{E}_{\max } \approx 210 \mathrm{eV}$. Presumably, the increase in $\delta_{\max }$ could be due to the elimination of water leaving purer aluminum, which has a higher SEY.

\section{3) Second crossover point}

The second crossover point, $\mathrm{E}_{\mathrm{II}}$, is an important parameter of the SEY curve. Figure 51 is an illustration of the SEY of an as-received air-exposed aluminum 6061 sample bombarded with a beam of electrons with energy spanning from $10 \mathrm{eV}$ to $1980 \mathrm{eV}$ in order to observe the second crossover point on the SEY curve. $\delta_{\max }$ is 1.50 at energy corresponding to $\mathrm{E}_{\max }$ $\approx 250 \mathrm{eV}$. The first crossover point is at $\mathrm{E}_{1}=30 \mathrm{eV}$ while the second crossover point is at $\mathrm{E}_{\mathrm{II}}=1580 \mathrm{eV}$. In this case, it can be again seen that the SEY of as-received air exposed aluminum sample is lower than the SEY of fresh as-received aluminum sample [37].

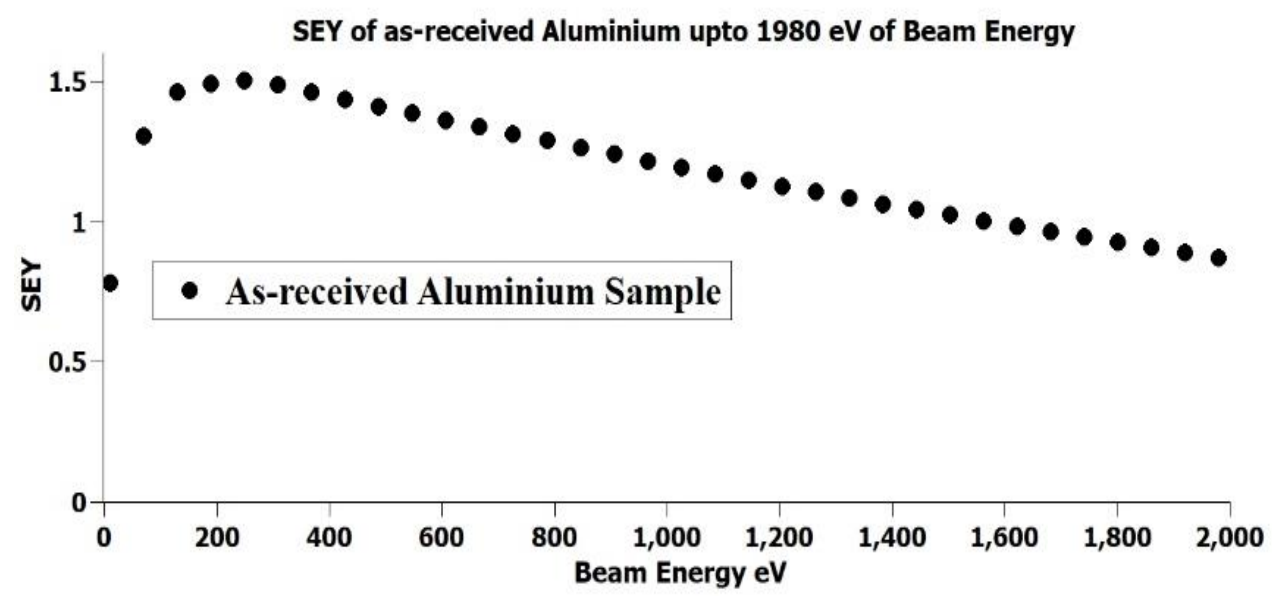

Figure 51: SEY OF AS-RECEIVED ALUMINIUM WITH BEAM ENERGY SCANNED UP TO 1980 EV. 
Figure 52 shows a comparison of the SEY of aluminum after employing different cleaning protocols.

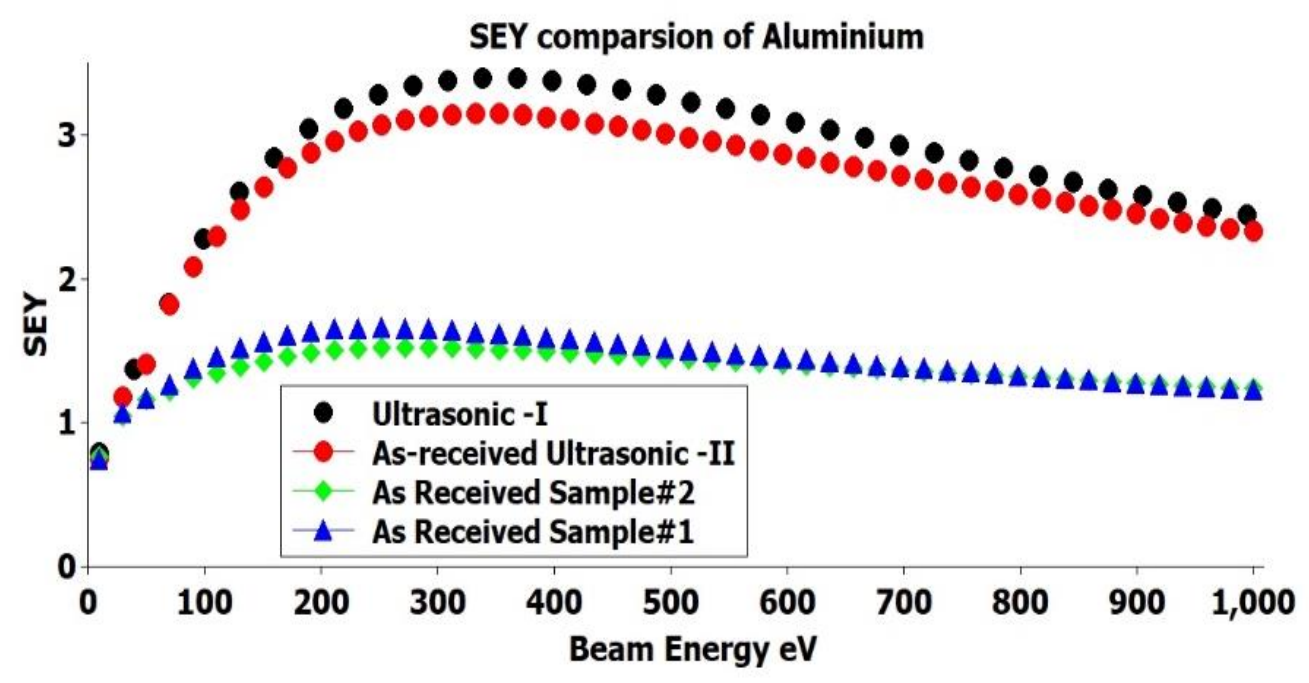

Figure 52: SEY COMPARISON OF ALUMINIUM WITH DIFFERENT SURFACE TREATMENTS.

The sample that was treated with the Ultrasonic-I protocol was a fresh sample received from the vendor. It showed a higher SEY with $\delta_{\max }=3.39$ with corresponding energy $\mathrm{E}_{\max }$ $\approx 350 \mathrm{eV}$. As can be seen, cleaning with the Ultrasonic-I protocol actually resulted in somewhat higher SEY, although still close to the SEY of the as-received sample. The sample that was treated with the ultrasonic-II protocol was as-received and air-exposed and resulted in $\delta_{\max }=3.14$ at corresponding $\mathrm{E}_{\max } \approx 350 \mathrm{eV}$. The Ultrasonic-II protocol reduced the SEY compared to Ultrasonic-I; however, it should be noted that the latter was not used on a fresh aluminum sample. The as-received, air-exposed sample shown previously had the lowest SEY of all the surface treatments tested on aluminum. 


\subsection{Comparison of experimental data with the "Universal Law" Model}

In this section, SEY experimental data are compared to the "Universal Law" analytical model given in [17]. A simplified, semiempirical analytical model of the SEY curve, the so called universal law, is derived from the classical empirical form of double power law.

$$
d \sim \mathrm{a}^{*} E^{n 1}+\mathrm{b}^{*} E^{n 2}
$$

where, $d=$ penetration depth of an incident electron

$$
\begin{aligned}
& E=\text { incident electron energy } \\
& n_{1}, n_{2}=\text { curve fitting parameters. }
\end{aligned}
$$

The simplified form of the SEY formula based on the variation of the stopping power with respect to the incident beam energy is expressed as

$$
\operatorname{SEY}(\delta)=d_{m} *\left(n_{2}-n_{1}\right) * \frac{1}{\left(n_{2}-1\right) *\left(\frac{x}{x_{m}}\right)^{\left(n_{1}-1\right)}-\left(n_{1}-1\right) *\left(\frac{x}{x_{m}}\right)^{\left(n_{2}-1\right)}},
$$

where the four parameters used as an input or starting point for curve fitting are

$$
\begin{aligned}
& d_{m}=\text { maximum SEY or } \delta_{\max } \\
& x_{m}=\text { Energy corresponding to } \mathrm{d}_{\mathrm{m}} \\
& \left(n_{1}-1\right) \text { and }\left(n_{2}-1\right)=\text { low and high energy asymptotes of the SEY curve, }
\end{aligned}
$$
respectively, on a log scale.

Figure 53 shows example curve fits of experimental data using Eq. (4.2) for copper that has undergone four different treatment methods. 


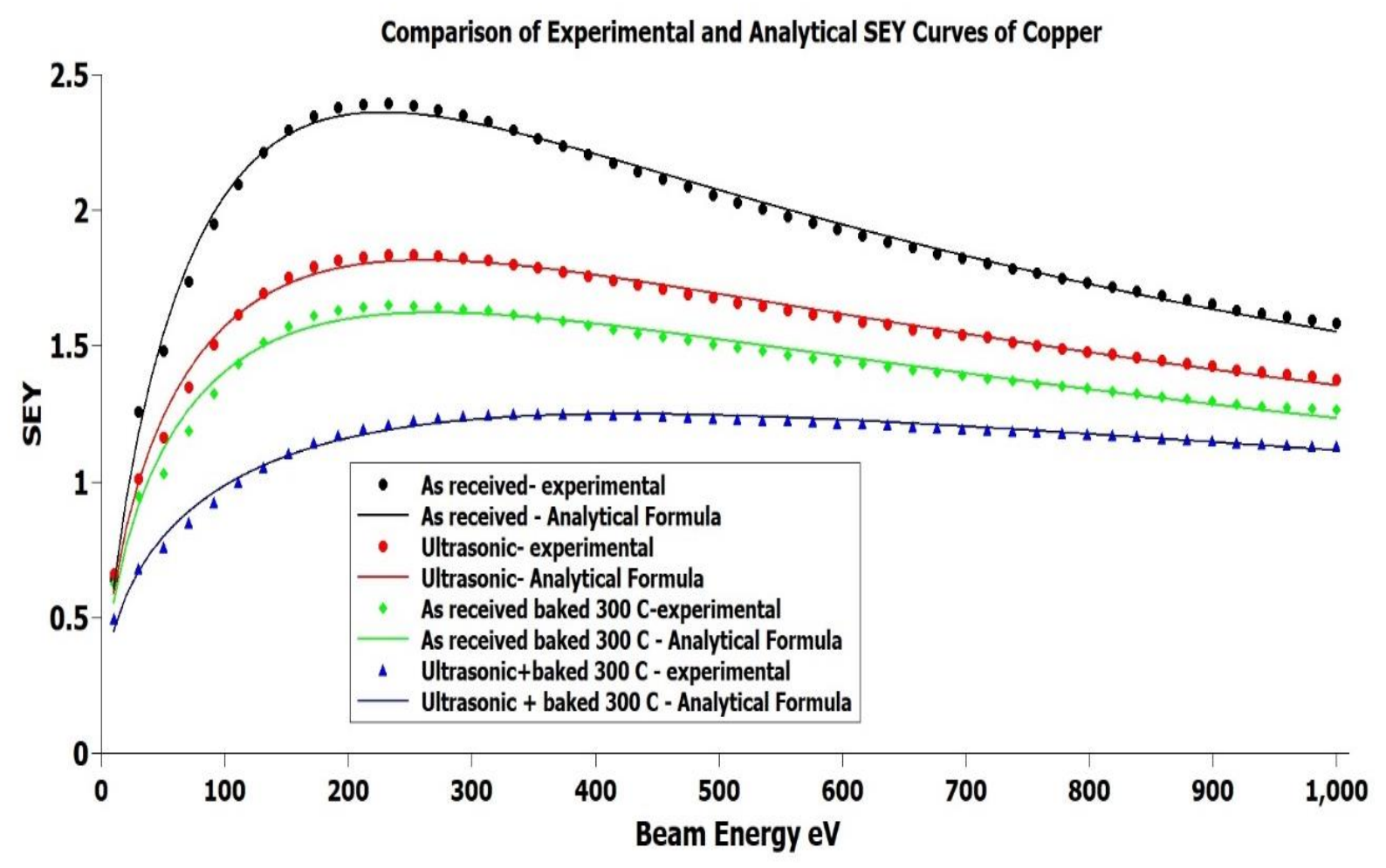

Figure 53: CURVE FITTING SEY EXPERIMENTAL DATA OF COPPER WITH UNIVERSAL LAW CURVE.

Solid lines on the graph represent analytical curves, while discrete data points show experimental measurement results. It can be seen in the figure that experimental data points fit reasonably well to the analytical form of SEY over the entire beam energy spectrum for all cleaning methods. In this case, parameters $\mathrm{n}_{1}$ and $\mathrm{n}_{2}$ are initially set to 1.5 and -1.5 respectively, but after fitting they are determined to be $n_{1}=1.67, n_{2}=0.35$ in the case of the as-received sample, $n_{1}=1.65, n_{2}=0.48$ in the case of the ultrasonically cleaned sample, $n_{1}=1.66, n_{2}=0.51$ in the case of the as-received and baked to $300{ }^{\circ} \mathrm{C}$, and $n_{1}=1.76, n_{2}=0.62$ in the case of the ultrasonically cleaned and baked to $300{ }^{\circ} \mathrm{C}$ sample.

Figure 54 shows another comparison of SEY experimental data with the analytical form for different surface treatment methods. 


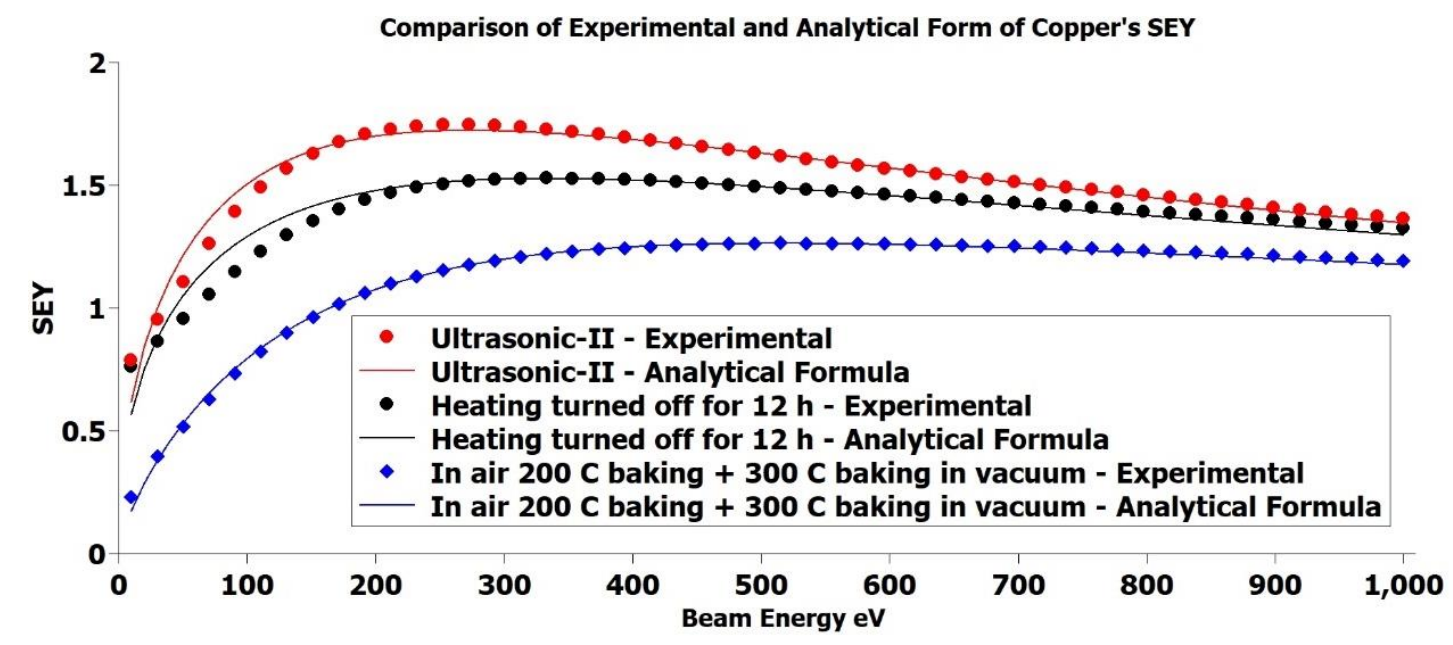

Figure 54: CURVE FITTING SEY EXPERIMENTAL DATA OF COPPER WITH UNIVERSAL LAW CURVE.

It can be seen in the figure that experimental data corresponding to the surface treatment method of in air + vacuum baking fits quite well to the analytical curve. The SEY experimental data corresponding to Ultrasonic-II and heating turned off for $12 \mathrm{hr}$ best fits the analytical form for electron energies greater than $200 \mathrm{eV}$. The analysis for curve fitting was performed using Qti plot software.

This curve fitting strategy is a good starting point to match SEY experimental data with SEY curves computed in simulations. The parameters $n_{1}$ and $n_{2}$ can be related to each surface treatment method. Further, if parameters $n_{1}$ and $n_{2}$ can be related to more detailed experimental parameters, such as surface adsorbates and sample elements, then the physics underlying these parameters might be elucidated through mathematical modeling. It might also be possible for a machine learning algorithm to be trained to predict SEY of materials of interest based on their composition and surface chemistry. 


\section{Chapter 5}

\section{Conclusions and Future Work}

\subsection{Conclusion}

An experimental system been established to perform SEY measurements as a function of beam energy (E), electron dose (D), angle of incidence of primary electrons $(\theta)$, and applied bias voltage (V). A number of materials of interest to high power RF VEDs were tested, including elemental metals like copper and silver, as well as alloys of aluminum 6061, invar, nickel-cobalt, and monel. Materials were cleaned using several surface treatment methods with the primary objective of identifying materials and cleaning protocols that could yield low SEY in order to suppress multipactor breakdown. The SEY of materials like copper and silver was also measured in the off-shelf state because SEY of off-shelf material are of interest to the aerospace industry, in addition to allowing comparisons with the SEY measured on cleaned materials.

Important conclusions derived from this experimental study are:

1. SEY is a surface-sensitive process. It depends on how the surface of the material is treated before introducing it into vacuum.

2. Reasonable levels of repeatability for identical measurements, with variations of $\leq$ $5 \%$ were achieved in the measurements presented in this thesis. There are many uncontrolled variables, such as exposure to air, contaminants present in the atmosphere, ambience temperature, cleanliness of the devices involved in 
Ultrasonic-I and Ultrasonic-II protocols, etc., that contribute to the variation in SEY.

3. Maximum repeatability is observed for SEY measurements taken after 12 hours of in-situ heating being turned off.

4. The dose effect plays a significant role in reducing the SEY, provided that the value of applied dose exceeds $10^{-6} \mathrm{C} / \mathrm{mm}^{2}$.

5. Beam conditioning the sample at low beam current did not reduce SEY.

6. Alloys tested in this thesis study showed higher SEY as compared to elementals metals.

7. Measurement of SEY, $\delta$, on copper showed that SEY increased with increasing angle of incidence.

8. It has been reported in the literature that most metal oxides have much higher SEY value than the corresponding pure metals. However, oxides of copper and aluminium tested in this work showed lower SEY than their corresponding "pure" metals.

9. The Ultrasonic-II cleaning protocol yielded lower SEY as compared to the Ultrasonic-I protocol because of better rinsing and cleaning. For the Ultrasonic-II protocol, diluted detergent and set up of three separate beakers filled with DI water were employed to increase water cavitation around samples for thorough removal of detergent residue.

10. Bakeouts under vacuum proved to be more efficacious than any other cleaning method. Baking to $300^{\circ} \mathrm{C}$ was more effective in reducing SEY than baking at $100^{\circ}$ $\mathrm{C}$ and $200^{\circ} \mathrm{C}$. 
11. Baking the copper sample to $300^{\circ} \mathrm{C}$ in vacuum for time duration $\geq 3$ hours along with ex-situ Ultrasonic-I,II or baking in air, shifts $\mathrm{E}_{\max }$ and $\mathrm{E}_{1}$ to higher electron beam energies.

12. The combination of ultrasonic-II cleaning and in situ bakifng to $300^{\circ} \mathrm{C}$ yielded the lowest SEY for copper and silver samples.

13. The universal curve fitting procedure provides good matches to the SEY of experimental data except for two cases of surface treatment methods as shown in chapter 4 (Fig. 54). Comparisons of universal curve fitting parameters may provide an avenue for better quantitative comparison with numerical modeling of SEY.

\subsection{Future Work}

The following is a list of topics and areas for continuing work.

1) A flowing water cleaning system is recommended to ensure efficient rinsing of the samples after ultrasonic cleaning with acetone and detergent.

2) The devices and containers involved in ultrasonic treatment should also be cleaned ultrasonically by using large capacity ultrasonic cleaners. This will be helpful in removing detergent suds and bubbles formed on the ultrasonic cleaners which contribute to adding impurity on the surface of material being cleaned.

3) Employ an experimental system that can allow heating to high temperature $\approx 1000^{\circ}$ C. In the current experimental scenario, the electron gun does not allow heating beyond $350^{\circ} \mathrm{C}$. Baking the sample at temperature around $1000^{\circ} \mathrm{C}$ will ensure removal of significantly more surface contaminants. 
4) Use in-situ X-ray photoelectron spectroscopy (XPS) surface analysis to determine the composition of the layers on the sample surface before and after the experiment.

5) Identify alloy materials with the help of MC simulations that could yield low SEY. The result of simulation could then be verified experimentally by testing those materials for SEY measurements.

6) Attach load lock chamber to reduce the cycle time of processing samples and it's also a clean method of introducing samples into vacuum chamber.

7) Variable leak valves can be installed on the vacuum chamber to feed various gases in the system in order to better characterize SEY with respect to surface chemistry. 


\section{References}

[1] J. R. M. Vaughan, "Multipactor," IEEE Transactions on electron devices, vol. 35, p. 1172-1180, 1988.

[2] R. A. Kishek, Y. Y. Lau, L. K. Ang, A. Valfells and R. M. Gilgenbach, "Multipactor discharge on metals and dielectrics: Historical review and recent theories," Physics of Plasmas, vol. 5, p. 2120-2126, 1998.

[3] T. P. Graves, "Standard/handbook for multipactor breakdown prevention in spacecraft components," 2014.

[4] V. E. Boria, L. Nuño, O. Monerris, D. Smacchia, D. Argilés and D. Raboso, "Experimental measurements and diagnosis of radio-frequency space high power and electromagnetic compatibility effects," in 2016 ESA Workshop on Aerospace EMC (Aerospace EMC), 2016.

[5] W. N. JohNsoN, R. Bartolo, M. Dorsey, D. Gubser, P. Jenkins, N. Smith, W. Boncyk, M. Brown, D. Huber and others, "Space-based solar power: Possible defense applications and opportunities for nrl contributions," 2009.

[6] S. Anza, C. Vicente and J. Gil, "RF breakdown analysis according to international standards," in 2016 IEEE International Conference on Plasma Science (ICOPS), 2016.

[7] W.-Z. Cui, Y. Li, J. Yang, T.-C. Hu, X.-B. Wang, R. Wang, N. Zhang, H.-T. Zhang and Y.-N. He, "An efficient multipaction suppression method in microwave components for space application," Chinese Physics B, vol. 25, p. 068401, 2016. 
[8] S. Michizono, "Secondary electron emission from alumina RF windows," IEEE

Transactions on Dielectrics and Electrical Insulation, vol. 14, p. 583-592, 2007.

[9] https://en.wikipedia.org/wiki/Multipactor_effect.

[10]_https://www.microwaves101.com/encyclopedias/multipaction.

[11] https://www.everythingrf.com/community/what-is-multi-paction.

[12] https://www.techbriefs.com/component/content/article/tb/features/articles/8274.

[13] O.Klemperer.Electron Physics: The physics of the Free Electron. Butterworth and Co (Publishers) Ltd, 88 Kingsway, London, WC2B 6AB,second edition,1972.

[14]https://www.physics-and-radio-electronics.com/electronic-devices-and-

circuits/electron-emission/thermionicemission.html.

[15] https://en.wikipedia.org/wiki/Field_electron_emission.

[16] D.Dukkamp J.J. Scholtz and R.W.A Schmitz. Secondary electron emission properties. Philips Journal of Research ,50(3/4):375-389,1996.

[17] S. Clerc, J. R. Dennison, R. Hoffmann and J. Abbott, "On the computation of secondary electron emission models," IEEE transactions on plasma science, vol. 34, p. 2219-2225, 2006.

[18] Jenkins, R.O.; Trodden, W.G. Electron and Ion Emission from Solids; Jacobs, L., Ed.; Routledge and Kegan Paul: London, UK, 1965; pp. 54-67.

[19] Nathan D. Zameroski. Effects of surface conditioning, morphology and temperature on secondary electron emission. M.S. Thesis, University of New Mexico, Albuquerque, NM, USA, May 2004. 
[20] J. Rodney M.Vaughan A new formula for secondary emission yield. IEEE Transaction on Electron Devices, 36(9):1963-1967,September 1989.

[21]Prasanth Kumar. Characterization of the Dose Effect in Secondary Electron Emission.M.S.Thesis,University of New Mexico,Alnuquerque,NM,USA,May,2007.

[22] H. Bruining, "Physics and applications of secondary electron emission," 1954.

[23] E.M. Baroody. A theory of secondary electron emission from metals. Physical Review, 78(6) : 780-787, June 1950.

[24] J.R. Young. Penetration of electrons in aluminum oxide films.Phys.Rev.,103(2):292293,Jul 1956.

[25] Gonzalez, L. A., Angelucci, M., Larciprete, R., \& Cimino, R. (2017). The secondary electron yield of noble metal surfaces. AIP Advances, 7, 115203.

[25] Gerald F.Dionne. Effects of secondary electron scattering on secondary emission yield curves. Journal of Applied Physics , 44(12) :5361 - 5364, December 1973.

[26] Gerald F.Dionne. Effects of secondary electron scattering on secondary emission yield curves. Journal of Applied Physics , 46(8) :3347 - 3351, August 1957.

[27] Arnold Shih and Charles Hor. Secondary emission properties as a function of the electron incidence angle. IEEE Transactions on Electron Devices, 40(4):824-828, April 1993.

[28] J. Rodney M.Vaughan Secondary electron emission formulas.IEEE Transaction on Electron Devices, 40(4):830,April 1993.

[29] https://www.everythingrf.com/community/what-is-a-twt-amplifier. 
[30] https://www.ukessays.com/essays/engineering/comparison-of-operation-of-twt-and-

magnetrons-engineering-essay.php

[31] https://en.wikipedia.org/wiki/Klystron.

[32] Gonzalez, L. A., Angelucci, M., Larciprete, R., \& Cimino, R. (2017). The secondary electron yield of noble metal surfaces. AIP Advances, 7, 115203.

[33] W. Kohn and L. J. Sham. "Self-consistent equations including exchange and correlation effects", Phys. Rev. 140, A1133 (1965).

[34] H. T. Nguyen-Truong, "Low-energy electron inelastic mean free path in materials," Applied Physics Letters, vol. 108, p. 172901, 2016.

[35] Dapor, M. (2012). Monte Carlo simulation of secondary electron emission from dielectric targets. Journal of Physics: Conference Series, 402, p. 012003.

[36] Z.-J. Ding and R. Shimizu, "A Monte Carlo modeling of electron interaction with solids including cascade secondary electron production," Scanning: The Journal of Scanning Microscopies, vol. 18, p. 92-113, 1996.

[37] V. Baglin, J. Bojko, C. Scheuerlein, O. Gröbner, M. Taborelli, B. Henrist and N. Hilleret, "The secondary electron yield of technical materials and its variation with surface treatments," 2000.

[38] J. Wang, Y. Wang, Y. Xu, B. Zhang and W. Wei, "Research on the secondary electron yield of TiZrV-Pd thin film coatings," Vacuum, vol. 131, p. 81-88, 2016.

[39] S. Wang, M. D. Cropper, O. B. Malyshev, R. Valizadeh and E. A. Seddon, "The secondary electron yield from transition metals," 2014. 
[40] F. L. Pimpec, F. King, R. E. Kirby and M. Pivi, "Secondary electron yield measurements of TiN coating and TiZrV getter film," arXiv preprint physics/0310071, 2003.

[41] H.-B. Zhang, X.-C. Hu, R. Wang, M. Cao, N. Zhang and W.-Z. Cui, "Note: Measuring effects of Ar-ion cleaning on the secondary electron yield of copper due to electron impact," Review of Scientific Instruments, vol. 83, p. 066105, 2012.

[42] M. Patino, Y. Raitses and R. Wirz, "Secondary electron emission from plasmagenerated nanostructured tungsten fuzz," Applied Physics Letters, vol. 109, p. 201602, 2016.

[43] I. Bojko, N. Hilleret and C. Scheuerlein, "Influence of air exposures and thermal treatments on the secondary electron yield of copper," Journal of Vacuum Science \& Technology A: Vacuum, Surfaces, and Films, vol. 18, p. 972-979, 2000.

[44] F. Le Pimpec, R. E. Kirby, F. K. King and M. Pivi, "Electron conditioning of technical aluminium surfaces: Effect on the secondary electron yield," Journal of Vacuum Science \& Technology A: Vacuum, Surfaces, and Films, vol. 23, p. 1610-1618, 2005.

[45] A. Iqbal, J. Ludwick, S. Fairchild, M. Cahay, D. Gortat, M. Sparkes, W. O’Neill, T. C. Back and P. Zhang, "Empirical modeling and Monte Carlo simulation of secondary electron yield reduction of laser drilled microporous gold surfaces," Journal of Vacuum Science \& Technology B, Nanotechnology and Microelectronics: Materials, Processing, Measurement, and Phenomena, vol. 38, p. 013801, 2020.

[46] John F.O’ Hanlon. A user's Guide to Vacuum Technology. Wiley-Interscience,2003. 
[47] Varian. TriScroll ${ }^{\mathrm{TM}} 300$ Series Dry Scroll Pump, Instruction Manual. Varian Vacuum Technologies, Lexington, Massachusetts, USA, Manual No.699904265, Feb 2005.

[48] Pfeiffer. TMH 260 C/ TMU 260 C and TMH 520 C/ TMU 520 C-Turbomolecular Drag Pumps for Corrosive Gas Processes, Instruction Manual. Pfeiffer Vacuum, Sagamore Park Road,Hudson,USA.

[49] https://www.idealvac.com/Pfeiffer-Balzers-TPH-190-Turbo-Molecular-HighVacuum-Pump-Rebuilt/pp/P102774.

[50] Kimball Physics. ELG-2 / EGPS-2 Electron Gun and Power Supply System. Kimball Physics Inc., Wilton, N.H., USA, 2003.

[51] Keithley. Model 6514 System Electrometer, Instruction Manual. Keithley Instruments, Inc., Cleveland, Ohio, USA, third printing edition, June 1999. [52] IDEC. Ry2ks series miniature magnetic latching relays. URL, http: // www. Idec .com /us / product support/ PDF/ U908_PDFs/ E_Relays/ E_Relays_RY2KS.pdf. [53] Tomi Engdahl. Simple circuit and program to show how to use pc parallel port output capabilities, URL, http :// www. smendes.com/e131p/parallel.htm,1996. [54] https://www.thinksrs.com/downloads/pdfs/manuals/RGAm.pdf.

[55] Ideal Vacuum weblink: https://www.idealvac.com/Electrical-ThermocoupleFeedthrough/pp/P108111.

[56] Department of Physics, Indiana University (HOM 2/1/00) [Rev. MRS: 10/4/11]. 
[57] E.W. McDaniel, “Collision Phenomenon in Ionized Gases”, John Wiley \& Sons, INC., New York -London - Sydney, 1964.

[58] R. Cimino, M. Commisso, D. R. Grosso, T. Demma, V. Baglin, R. Flammini and R. Larciprete, "Nature of the decrease of the secondary-electron yield by electron bombardment and its energy dependence," Physical review letters, vol. 109, p. 064801, 2012. 